\title{
Enantioenriched 1-Tetralones via Rhodium-Catalyzed Arylative Cascade Desymmetrization/Acylation of Alkynylmalonates
}

Aymane Selmani, Sylvain Darses*

PSL Université Paris, Chimie ParisTech - CNRS, Institute of Chemistry for Life and Health Sciences (i-CLeHS), 11 rue Pierre et Marie Curie, 75005, Paris (France)

\section{Supporting Information}

\section{Table of contents}

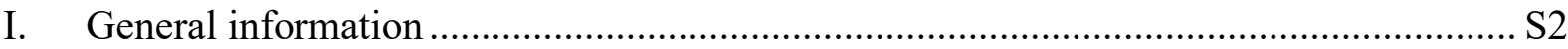

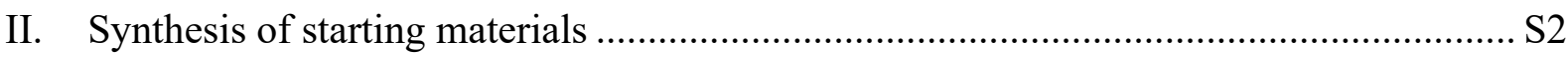

A. General procedure (1) for the preparation of starting materials......................... S2

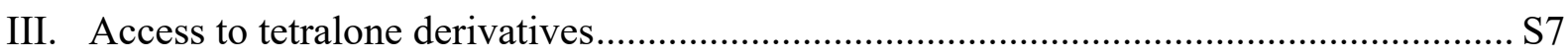

A. General procedure (2) for the preparation of racemic tetralone derivatives ......... S7

B. General procedure (3) for the preparation of chiral tetralone derivatives.............S7

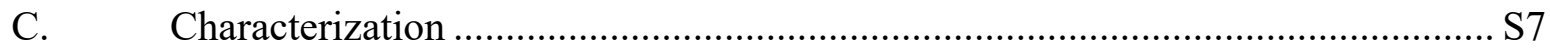

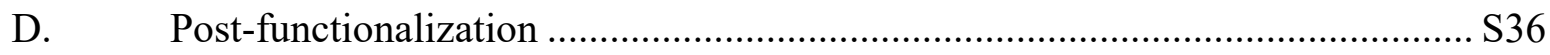

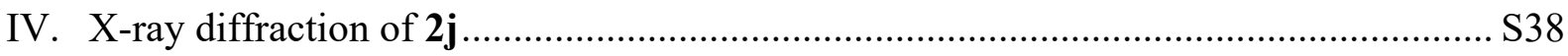

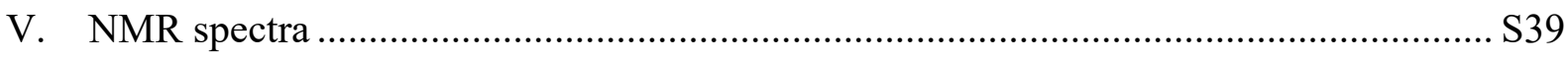




\section{General information}

All reactions were performed under an argon atmosphere. Reaction vessels were oven-dried, cooled under vacuum and flushed with argon before use. $\mathrm{MeOH}$ was distilled over sodium, water was distilled, DCM and THF was dried over alumina columns in an Innovative Technologies apparatus. All the solvents for catalysis were degassed prior to use. Reagents were obtained from commercial suppliers and used without further purification.

Reactions were monitored by thin layer chromatography (TLC) carried out on silica gel plates (Merck Kieselgel 60 F254); spots were detected with UV light and/or by staining with $\mathrm{KMnO}_{4}$ solution. Flash column chromatography was performed on silica gel (Merck, spherical, neutral, 40-60 $\mu \mathrm{m})$.

${ }^{1} \mathrm{H}$ NMR, ${ }^{19} \mathrm{~F}$ NMR and ${ }^{13} \mathrm{C}\left\{{ }^{1} \mathrm{H}\right\}$ NMR were recorded on a Bruker Avance 400 instrument in $\mathrm{CDCl}_{3}$. Chemical shifts are reported in delta $(\delta)$ units part per million (ppm). Residual $\mathrm{CHCl}_{3}$ $\left(\delta\right.$ 7.26) and $\mathrm{CDCl}_{3}\left(\delta\right.$ 77.0) were used as internal standards for ${ }^{1} \mathrm{H}$ and ${ }^{13} \mathrm{C}$ NMR spectra respectively. Coupling constants $(J)$ are reported in Hertz $(\mathrm{Hz})$. The following abbreviations are used: s, singlet, d, doublet, t, triplet, q, quartet, h, heptulet, m, multiplet, br, broad.

Enantiomeric excesses (ee) were determined by HPLC analyses using a Waters alliance e2695 system equipped with chiral stationary phase columns Daicel Chiralpak coupled with a dual wavelength $(215 / 254 \mathrm{~nm})$ Waters 2489 UV detector. Optical rotations were measured on a Perkin-Elmer 241 polarimeter $(\lambda=589 \mathrm{~nm}, \mathrm{Na}$ lamp, $1 \mathrm{dm}$ cell). High-resolution mass spectroscopic (HRMS) analyses were measured on LTQOrbitrap (Thermo Fisher Scientific) at Pierre et Marie Curie University. Melting points were measured with a Stuart Scientific melting point apparatus SMP1.

X-ray diffraction was made at Pierre et Marie Curie University. CCDC 1926608 contains the supplementary crystallographic data for compound $\mathbf{2} \mathbf{j}$. These data can be obtained free of charge from The Cambridge Crystallographic Data Centre via www.ccdc.cam.ac.uk/data request/cif.

\section{Synthesis of starting materials}

\section{A. General procedure (1) for the preparation of starting materials}

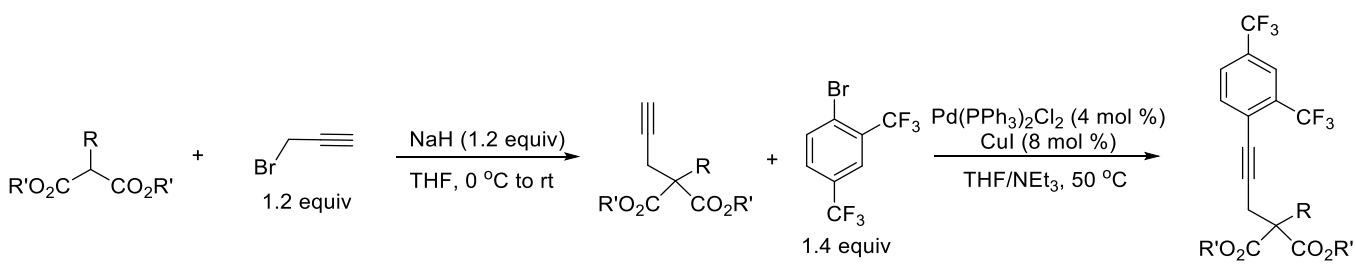

To a solution of alkylmalonate ( 1 equiv) in dry THF was added at $0{ }^{\circ} \mathrm{C}$ sodium hydride $(1.2$ equiv) under argon. The mixture was stirred at room temperature for $0.5 \mathrm{~h}$, then propargyl bromide (1.2 equiv) was added and the reaction was stirred at room temperature overnight. The mixture was diluted with aq. $\mathrm{HCl} 1 \mathrm{M}$ and extracted with ethyl acetate. Combined organic layers were washed with brine, dried over $\mathrm{MgSO}_{4}$, filtered and concentrated under vacuum. In a Schlenk tube, equipped with a magnetic stirring bar, were introduced the crude mixture, $\mathrm{PdCl}_{2}\left(\mathrm{PPh}_{3}\right)_{2}(4 \mathrm{~mol} \%), \mathrm{CuI}(8 \mathrm{~mol} \%)$ and THF. The tube was evacuated under vacuum and placed under argon atmosphere. Then, 2,4-bis(trifluoromethyl)bromobenzene (1.4 equiv) and $\mathrm{Et}_{3} \mathrm{~N}$ were added successively under an argon atmosphere. The resulting mixture was stirred at $50{ }^{\circ} \mathrm{C}$ until full conversion. The reaction mixture was diluted with water and extracted with ethyl acetate. Combined organic layers were washed with brine, dried over $\mathrm{MgSO}_{4}$, filtered and concentrated under vacuum. The crude was purified by flash chromatography on silica gel. 
Diethyl 2-benzyl-2-(3-(2,4-bis(trifluoromethyl)phenyl)prop-2-yn-1-yl)malonate $\left(\mathrm{S}_{1}\right)$<smiles>CCOC(=O)C(CC#Cc1ccc(C(F)(F)F)cc1C(F)(F)F)(Cc1ccccc1)OCC</smiles>

Orange oil (4.3 g, $85 \%$ ) obtained from the general procedure (1) using diethyl benzylmalonate $(2.5 \mathrm{~g}, 10.0 \mathrm{mmol})$.

$\mathbf{R}_{\mathbf{f}}=0.37$ (petroleum ether/EtOAc 9:1).

${ }^{1} \mathbf{H}$ NMR $\left(400 \mathrm{MHz}, \mathrm{CDCl}_{3}, \delta\right): 7.90(\mathrm{~s}, 1 \mathrm{H}), 7.75(\mathrm{~d}, J=8.2 .1 .8 \mathrm{~Hz}, 1 \mathrm{H}), 7.71(\mathrm{~d}, J=8.2 \mathrm{~Hz}$, $1 \mathrm{H}), 7.33-7.24(\mathrm{~m}, 3 \mathrm{H}), 7.23-7.15(\mathrm{~m}, 2 \mathrm{H}), 4.66-4.04(\mathrm{~m}, 4 \mathrm{H}), 3.45(\mathrm{~s}, 2 \mathrm{H}), 2.98(\mathrm{~s}, 2 \mathrm{H}), 1.27$ (t, $J=7.1 \mathrm{~Hz}, 6 \mathrm{H})$.

${ }^{13}$ C NMR (101 MHz, $\left.\mathrm{CDCl}_{3}, \delta\right): 169.4(2 \mathrm{C}), 135.5,135.0,132.2$ (q, $\left.J=31.3 \mathrm{~Hz}\right), 129.9$ (q, $J$ $=33.5 \mathrm{~Hz}), 129.9(2 \mathrm{C}), 128.4(2 \mathrm{C}), 128.2(\mathrm{q}, J=3.6 \mathrm{~Hz}), 127.2,125.4,123.2(\mathrm{q}, J=272.5$ $\mathrm{Hz}), 123.0$ (hept, $J=4.7 \mathrm{~Hz}), 122.8$ (q, $J=273.6 \mathrm{~Hz}), 94.4,78.9,61.8$ (2C), 58.3, 37.5, 23.3, $14.0(2 \mathrm{C})$.

${ }^{19}$ F NMR $\left(376 \mathrm{MHz}, \mathrm{CDCl}_{3}, \delta\right):-62.7,-63.0$.

Dimethyl 2-benzyl-2-(3-(2,4-bis(trifluoromethyl)phenyl)prop-2-yn-1-yl)malonate $\left(\mathbf{S}_{2}\right)$<smiles>CC(=O)C(CC#Cc1ccc(C(F)(F)F)cc1C(F)(F)F)(Cc1ccccc1)C(C)(F)F</smiles>

Orange oil (1.19 g, $72 \%)$ obtained from the general procedure (1) using dimethyl benzylmalonate (777 mg, $3.5 \mathrm{mmol}$ ).

$\mathbf{R}_{\mathbf{f}}=0.32$ (petroleum ether/EtOAc 9:1).

${ }^{1} \mathbf{H}$ NMR $\left(400 \mathrm{MHz}, \mathrm{CDCl}_{3}, \delta\right): 7.91(\mathrm{~s}, 1 \mathrm{H}), 7.75(\mathrm{dd}, J=8.2,1.8 \mathrm{~Hz}, 1 \mathrm{H}), 7.72(\mathrm{~d}, J=8.2$ $\mathrm{Hz}, 1 \mathrm{H}), 7.38-7.21(\mathrm{~m}, 3 \mathrm{H}), 7.22-7.14(\mathrm{~m}, 2 \mathrm{H}), 3.78(\mathrm{~s}, 6 \mathrm{H}), 3.47(\mathrm{~s}, 2 \mathrm{H}), 3.00(\mathrm{~s}, 2 \mathrm{H})$.

${ }^{13}$ C NMR (101 MHz, $\mathrm{CDCl}_{3}, \delta$ ): 169.9 (2C), 135.4, 135.0, 132.3 (q, $J=31.6 \mathrm{~Hz}$ ), 130.3 (q, $J$ $=33.7 \mathrm{~Hz}), 129.8(2 \mathrm{C}), 128.5(2 \mathrm{C}), 128.3(\mathrm{q}, J=3.8 \mathrm{~Hz}), 127.3,125.3,123.2(\mathrm{q}, J=272.6$ $\mathrm{Hz}), 123.0$ (hept, $J=3.5 \mathrm{~Hz}), 122.8(\mathrm{q}, J=273.7 \mathrm{~Hz}), 94.2,79.0,58.4,52.8(2 \mathrm{C}), 37.8,23.4$.

${ }^{19}$ F NMR $\left(376 \mathrm{MHz}, \mathrm{CDCl}_{3}, \delta\right):-62.7,-63.0$.

Diisopropyl 2-benzyl-2-(3-(2,4-bis(trifluoromethyl)phenyl)prop-2-yn-1-yl)malonate $\left(\mathbf{S}_{3}\right)$ 
<smiles>CCCOC(CC#Cc1ccc(C(F)(F)F)cc1C(F)(F)F)(Cc1ccccc1)C(=O)OC(C)C</smiles>

Orange oil (792 mg, $50 \%$ ) obtained from the same procedure using diisopropyl benzylmalonate (834 mg, $3.0 \mathrm{mmol}$ ).

$\mathbf{R}_{\mathbf{f}}=0.45$ (petroleum ether/EtOAc 9:1).

${ }^{1} \mathbf{H}$ NMR $\left(400 \mathrm{MHz}, \mathrm{CDCl}_{3}, \delta\right): 7.90(\mathrm{~s}, 1 \mathrm{H}), 7.75(\mathrm{dd}, J=8.1,1.8 \mathrm{~Hz}, 1 \mathrm{H}), 7.70(\mathrm{~d}, J=8.1$ $\mathrm{Hz}, 1 \mathrm{H}), 7.36-7.16(\mathrm{~m}, 5 \mathrm{H}), 5.08$ (hept, $J=6.2 \mathrm{~Hz}, 2 \mathrm{H}), 3.42(\mathrm{~s}, 2 \mathrm{H}), 2.96$ (s, 2H), 1.29 (d, $J$ $=6.3 \mathrm{~Hz}, 6 \mathrm{H}), 1.23(\mathrm{~d}, J=6.2 \mathrm{~Hz}, 6 \mathrm{H})$.

${ }^{13}$ C NMR (101 MHz, $\left.\mathrm{CDCl}_{3}, \delta\right): 169.0(2 \mathrm{C}), 135.7,135.0,132.2$ (q, $\left.J=31.3 \mathrm{~Hz}\right), 130.0(2 \mathrm{C})$, $129.9(\mathrm{q}, J=33.2 \mathrm{~Hz}), 128.4(2 \mathrm{C}), 128.2(\mathrm{q}, J=3.6 \mathrm{~Hz}), 127.2,125.5,123.1$ (hept, $J=4.1$ Hz), 123.2 (q, $J=272.1 \mathrm{~Hz}), 122.8$ (q, $J=273.8 \mathrm{~Hz}), 94.6,78.9,69.5$ (2C), 58.2, 37.3, 23.3, $21.6(2 \mathrm{C}), 21.5(2 \mathrm{C})$.

${ }^{19}$ F NMR (376 MHz, $\left.\mathrm{CDCl}_{3}, \delta\right):-62.7,-63.0$.

Dimethyl 2-(3-(2,4-bis(trifluoromethyl)phenyl)prop-2-yn-1-yl)-2-methylmalonate $\left(\mathrm{S}_{4}\right)$<smiles>COC(C)C(C)(CC#Cc1ccc(C(F)(F)F)cc1C(F)(F)F)OC</smiles>

Orange oil (796 mg, $67 \%$ ) obtained from the same procedure using dimethyl methylmalonate (438 mg, $3.0 \mathrm{mmol})$.

$\mathbf{R}_{\mathbf{f}}=0.34$ (petroleum ether/EtOAc 9:1).

${ }^{1}$ H NMR $\left(400 \mathrm{MHz}, \mathrm{CDCl}_{3}, \delta\right): 7.86(\mathrm{~s}, 1 \mathrm{H}), 7.72(\mathrm{dd}, J=8.1,1.8 \mathrm{~Hz}, 1 \mathrm{H}), 7.65(\mathrm{~d}, J=8.1$ $\mathrm{Hz}, 1 \mathrm{H}), 3.77$ (s, 6H), $3.10(\mathrm{~s}, 2 \mathrm{H}), 1.62(\mathrm{~s}, 3 \mathrm{H})$.

${ }^{13}$ C NMR (101 MHz, $\left.\mathrm{CDCl}_{3}, \delta\right): 171.1(2 \mathrm{C}), 134.8,132.2$ (q, $\left.J=31.4 \mathrm{~Hz}\right), 129.9$ (q, $J=33.9$ $\mathrm{Hz}), 128.2$ (q, $J=3.8 \mathrm{~Hz}), 125.4,123.2(\mathrm{q}, J=272.6 \mathrm{~Hz}), 122.9$ (hept, $J=4.5 \mathrm{~Hz}), 122.7$ (q, $J=273.6 \mathrm{~Hz}), 94.2,78.3,53.3,52.9(2 \mathrm{C}), 27.1,20.0$.

${ }^{19}$ F NMR $\left(376 \mathrm{MHz}, \mathrm{CDCl}_{3}, \delta\right):-62.9,-63.1$.

Dimethyl 2-(3-(2,4-bis(trifluoromethyl)phenyl)prop-2-yn-1-yl)-2-phenethylmalonate $\left(\mathbf{S}_{5}\right)$<smiles>COC(=O)C(CC#Cc1ccc(C(F)(F)F)cc1C(F)(F)F)(CCc1ccccc1)C(C)=O</smiles> 
Orange oil (1.4 g, $71 \%$ ) obtained from the same procedure using dimethyl 2-phenethylmalonate (944 mg, $4.0 \mathrm{mmol})$.

$\mathbf{R}_{\mathbf{f}}=0.27$ (petroleum ether/EtOAc 9:1).

${ }^{1}$ H NMR $\left(400 \mathrm{MHz}, \mathrm{CDCl}_{3}, \delta\right): 7.87(\mathrm{~s}, 1 \mathrm{H}), 7.72(\mathrm{dd}, J=8.2,2.1 \mathrm{~Hz}, 1 \mathrm{H}), 7.64(\mathrm{~d}, J=8.2$ $\mathrm{Hz}, 1 \mathrm{H}), 7.31-7.27(\mathrm{~m}, 3 \mathrm{H}), 7.22-7.14(\mathrm{~m}, 2 \mathrm{H}), 3.76(\mathrm{~s}, 6 \mathrm{H}), 3.22(\mathrm{~s}, 2 \mathrm{H}), 2.62-2.55(\mathrm{~m}, 2 \mathrm{H})$, 2.49-2.37 (m, 2H).

${ }^{13}$ C NMR (101 MHz, $\mathrm{CDCl}_{3}, \delta$ ): 170.4 (2C), 140.8, 134.9, 132.3 (q, $J=31.6 \mathrm{~Hz}$ ), 129.9 (q, $J$ $=33.6 \mathrm{~Hz}), 128.5(2 \mathrm{C}), 128.4(2 \mathrm{C}), 128.2(\mathrm{q}, J=3.6 \mathrm{~Hz}), 126.2,125.3,123.2(\mathrm{q}, J=272.3$ $\mathrm{Hz}$ ), 123.0 (hept, $J=3.7 \mathrm{~Hz}), 122.7$ (q, $J=274.1 \mathrm{~Hz}), 93.8,78.4,57.0,52.9$ (2C), 34.1, 30.6, 24.2 .

${ }^{19}$ F NMR $\left(376 \mathrm{MHz}, \mathrm{CDCl}_{3}, \delta\right):-62.8,-63.0$.

Diethyl 2-(3-(2,4-bis(trifluoromethyl)phenyl)prop-2-yn-1-yl)-2-butylmalonate $\left(\mathbf{S}_{6}\right)$

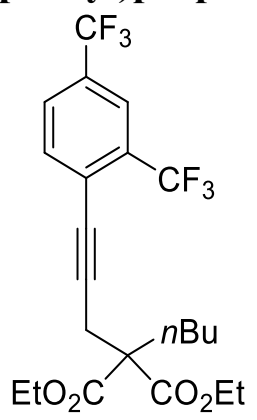

Orange oil (1.6 g, $76 \%$ ) obtained from the same procedure using diethyl 2-butylmalonate (972 $\mathrm{mg}, 4.5 \mathrm{mmol})$.

$\mathbf{R}_{\mathbf{f}}=0.40$ (petroleum ether/EtOAc 9:1).

${ }^{1}$ H NMR $\left(400 \mathrm{MHz}, \mathrm{CDCl}_{3}, \delta\right): 7.86(\mathrm{~s}, 1 \mathrm{H}), 7.71(\mathrm{dd}, J=8.1,1.9 \mathrm{~Hz}, 1 \mathrm{H}), 7.63(\mathrm{~d}, J=8.1$ $\mathrm{Hz}, 1 \mathrm{H}), 4.22(\mathrm{q}, J=7.1 \mathrm{~Hz}, 4 \mathrm{H}), 3.12(\mathrm{~s}, 2 \mathrm{H}), 2.14-2.05(\mathrm{~m}, 2 \mathrm{H}), 1.47-1.30(\mathrm{~m}, 2 \mathrm{H}), 1.26(\mathrm{t}$, $J=7.1 \mathrm{~Hz}, 6 \mathrm{H}), 1.23-1.16(\mathrm{~m}, 2 \mathrm{H}), 0.92(\mathrm{t}, J=7.1 \mathrm{~Hz}, 3 \mathrm{H})$.

${ }^{13} \mathrm{C}$ NMR (101 MHz, $\left.\mathrm{CDCl}_{3}, \delta\right): 170.2(2 \mathrm{C}), 134.9,132.2(\mathrm{q}, J=31.5 \mathrm{~Hz}), 129.8(\mathrm{q}, J=33.5$ $\mathrm{Hz}), 128.1$ (q, $J=3.5 \mathrm{~Hz}), 125.5,123.2$ (q, $J=272.2 \mathrm{~Hz}), 123.0$ (hept, $J=4.2 \mathrm{~Hz}$ ), 122.7 (q, $J=273.8 \mathrm{~Hz}), 94.3,78.1,61.6(2 \mathrm{C}), 57.0,31.7,26.1,23.8,22.8,14.0$ (2C), 13.8 .

${ }^{19}$ F NMR $\left(376 \mathrm{MHz}, \mathrm{CDCl}_{3}, \delta\right):-62.9,-63.0$.

Diethyl

2-(3-(2,4-bis(trifluoromethyl)phenyl)prop-2-yn-1-yl)-2-((tertbutoxycarbonyl)amino)malonate $\left(\mathbf{S}_{7}\right)$<smiles>CCOC(CC#Cc1ccc(C(F)(F)F)cc1C(F)(F)F)(NC(C)(CC)C(F)(F)F)OCC</smiles>

Orange oil (1.6 g, $64 \%$ ) obtained from the same procedure using diethyl (boc-amino)malonate (1.3 g, $4.7 \mathrm{mmol})$.

$\mathbf{R}_{\mathbf{f}}=0.25$ (petroleum ether/EtOAc 9:1).

${ }^{1} \mathbf{H}$ NMR $\left(400 \mathrm{MHz}, \mathrm{CDCl}_{3}, \delta\right): 7.86(\mathrm{~s}, 1 \mathrm{H}), 7.72(\mathrm{dd}, J=8.1,1.6 \mathrm{~Hz}, 1 \mathrm{H}), 7.64(\mathrm{~d}, J=8.1$ $\mathrm{Hz}, 1 \mathrm{H}), 6.14(\mathrm{~s}, 1 \mathrm{H}), 4.47-4.11(\mathrm{~m}, 4 \mathrm{H}), 3.56(\mathrm{~s}, 2 \mathrm{H}), 1.45$ (s, 9H), 1.27 (t, J=7.1 Hz, 6H). 
${ }^{13}$ C NMR (101 MHz, $\left.\mathrm{CDCl}_{3}, \delta\right): 166.7$ (2C), 154.0, 134.8, 132.4 (q, $\left.J=31.2 \mathrm{~Hz}\right), 129.9$ (q, $J$ $=33.9 \mathrm{~Hz}), 128.1(\mathrm{q}, J=3.2 \mathrm{~Hz}), 125.4,123.2(\mathrm{q}, J=272.4 \mathrm{~Hz}), 122.9$ (hept, $J=4.2 \mathrm{~Hz}$ ), 122.7 (q, $J=273.7 \mathrm{~Hz}), 93.5,80.6,78.3,65.5,62.9(2 \mathrm{C}), 28.2$ (3C), 25.5, 13.9 (2C).

${ }^{19}$ F NMR $\left(376 \mathrm{MHz}, \mathrm{CDCl}_{3}, \delta\right):-62.9,-63.0$.

Diethyl 2-acetamido-2-(3-(2,4-bis(trifluoromethyl)phenyl)prop-2-yn-1-yl)malonate (S8)<smiles>CCOC(OCC)C(CC#Cc1ccc(C(F)(F)F)cc1C(F)(F)F)(NC(C)(F)F)OCC</smiles>

Orange oil (5.1 g, $76 \%)$ obtained from the same procedure using diethyl acetamidomalonate $(3.0 \mathrm{~g}, 14.4 \mathrm{mmol})$.

$\mathbf{R}_{\mathbf{f}}=0.35$ (petroleum ether/EtOAc 6:4).

${ }^{1} \mathbf{H}$ NMR $\left(400 \mathrm{MHz}, \mathrm{CDCl}_{3}, \delta\right): 7.86(\mathrm{~s}, 1 \mathrm{H}), 7.72(\mathrm{dd}, J=8.1,1.1 \mathrm{~Hz}, 1 \mathrm{H}), 7.62(\mathrm{~d}, J=8.1$ $\mathrm{Hz}, 1 \mathrm{H}), 6.98(\mathrm{~s}, 1 \mathrm{H}), 4.28(\mathrm{q}, J=7.1 \mathrm{~Hz}, 4 \mathrm{H}), 3.60(\mathrm{~s}, 2 \mathrm{H}), 2.07(\mathrm{~s}, 3 \mathrm{H}), 1.27(\mathrm{t}, J=7.1 \mathrm{~Hz}$, $6 \mathrm{H})$.

${ }^{13} \mathbf{C}$ NMR (101 MHz, $\mathrm{CDCl}_{3}, \delta$ ): 169.4 (2C), 166.5, 134.8, 132.2 (q, $J=31.2 \mathrm{~Hz}$ ), 130.0 (q, $J$ $=33.6 \mathrm{~Hz}), 128.3(\mathrm{q}, J=3.6 \mathrm{~Hz}), 125.2,123.1(\mathrm{q}, J=272.9 \mathrm{~Hz}), 123.0(\mathrm{q}, J=4.3 \mathrm{~Hz}), 122.8$ (q, $J=273.7 \mathrm{~Hz}), 93.3,78.3,65.2,63.1(2 \mathrm{C}), 24.9,22.8,13.9$ (2C).

${ }^{19}$ F NMR $\left(376 \mathrm{MHz}, \mathrm{CDCl}_{3}, \delta\right):-62.8,-63.1$. 


\section{Access to tetralone derivatives}

\section{A. General procedure (2) for the preparation of racemic tetralone derivatives}

In a septum-capped vial, equipped with a magnetic stirring bar, were introduced $[\mathrm{Rh}(\operatorname{cod}) \mathrm{OH}]_{2}$ $1.5 \mathrm{~mol} \%, 2.25 \mu \mathrm{mol}, 1.1 \mathrm{mg})$, the corresponding boronic acid $(0.30 \mathrm{mmol})$ and malonate derivative $(0.15 \mathrm{mmol})$. The vial was closed, evacuated under vacuum and placed under argon atmosphere. Degassed $\mathrm{MeOH}$ was added and the resulting mixture was heated at $60{ }^{\circ} \mathrm{C}$ (preheated oil bath) until full consumption of the starting material. Solvents were removed under vacuum and the crude was purified by silica gel chromatography.

\section{B. General procedure (3) for the preparation of chiral tetralone derivatives}

In a septum-capped vial, equipped with a magnetic stirring bar, were introduced the chiral diene anthracen9-yl-MSBod $(3.3 \mathrm{~mol} \%, 10.0 \mu \mathrm{mol}, 3.4 \mathrm{mg})$ and $\left[\mathrm{Rh}\left(\mathrm{C}_{2} \mathrm{H}_{4}\right)_{2} \mathrm{Cl}\right]_{2}(1.5 \mathrm{~mol} \%, 4.5$ $\mu \mathrm{mol}, 1.8 \mathrm{mg})$. The vial was closed, evacuated under vacuum and placed under argon atmosphere. Degassed dichloromethane $(0.2 \mathrm{~mL})$ was added and the mixture was stirred for 15 min at room temperature. Then, a degassed solution of $\mathrm{KOH}$ in $\mathrm{MeOH}(50 \mu \mathrm{L}, \mathrm{c}=0.22 \mathrm{M})$ was added, the solution was stirred for $15 \mathrm{~min}$ and solvents were removed under vacuum. The corresponding boronic acid $(0.30 \mathrm{mmol})$ and the malonate derivative $(0.15 \mathrm{mmol})$ were added to the dry residue, followed by degassed methanol $(0.5 \mathrm{~mL})$ and the resulting mixture was heated at $60^{\circ} \mathrm{C}$ (preheated oil bath) until full conversion of the starting material. Solvents were removed under vacuum and the crude was purified by silica gel chromatography.

\section{Characterization}

Ethyl (R,E)-2-benzyl-4-(2,4-bis(trifluoromethyl)benzylidene)-1-oxo-1,2,3,4tetrahydronaphthalene-2-carboxylate (2a)<smiles>CCOC1(Cc2ccccc2)CC(=Cc2ccc(C(F)(F)F)cc2C(F)(F)F)c2ccccc2C1=O</smiles>

Yellow oil (75 mg, $94 \%$ ) obtained from the general procedure (3) using $\mathbf{S}_{\mathbf{1}}$ (75 mg, $0.15 \mathrm{mmol}$ ) and phenylboronic acid (37 $\mathrm{mg}, 0.30 \mathrm{mmol}$ ).

$\mathbf{R}_{\mathbf{f}}=0.38$ (petroleum ether/EtOAc 9:1).

HPLC (Chiralpak IC hexane $/ \mathrm{iPrOH} 97: 3,0.9 \mathrm{~mL} / \mathrm{min}$ ): $\mathrm{Rt}=10.5 \mathrm{~min}$ (major), $11.4 \mathrm{~min}$ (minor).

$[\boldsymbol{\alpha}]_{\mathbf{D}} \mathbf{2 0}^{\mathbf{2 0}}=-180\left(\mathrm{c}=2.4, \mathrm{CHCl}_{3}\right)$ for an enantiomeric excess of $85 \%$.

${ }^{1} \mathbf{H}$ NMR $\left(400 \mathrm{MHz} \mathrm{CDCl}_{3}, \delta\right): 8.15(\mathrm{dd}, J=7.8,1.5 \mathrm{~Hz}, 1 \mathrm{H}), 8.04-7.97(\mathrm{~m}, 1 \mathrm{H}), 7.86-7.78$ $(\mathrm{m}, 1 \mathrm{H}), 7.74(\mathrm{dd}, J=8.1,1.1 \mathrm{~Hz}, 1 \mathrm{H}), 7.67-7.57(\mathrm{~m}, 2 \mathrm{H}), 7.54-7.37(\mathrm{~m}, 2 \mathrm{H}), 7.20-7.11(\mathrm{~m}$, $5 \mathrm{H}), 4.06$ (hept, $J=7.1 \mathrm{~Hz}, 2 \mathrm{H}), 3.49$ (d, $J=13.7 \mathrm{~Hz}, 1 \mathrm{H}), 3.35$ (dd, $J=14.5,2.1 \mathrm{~Hz}, 1 \mathrm{H})$, $3.19(\mathrm{~d}, J=13.7 \mathrm{~Hz}, 1 \mathrm{H}), 2.67(\mathrm{dd}, J=14.5,2.1 \mathrm{~Hz}, 1 \mathrm{H}), 1.05(\mathrm{t}, J=7.1 \mathrm{~Hz}, 3 \mathrm{H})$. 
${ }^{13}$ C NMR (101 MHz, $\left.\mathrm{CDCl}_{3}, \delta\right):$ 193.6, 170.9, 139.6, 139.2, 136.0, 135.5, 134.0, 131.7, 131.3, $130.7(2 \mathrm{C}), 129.8$ (q, $J=33.6 \mathrm{~Hz}), 129.7(\mathrm{q}, J=30.7 \mathrm{~Hz}), 129.2,128.4(\mathrm{q}, J=3.6 \mathrm{~Hz}), 128.3$, $128.1(2 \mathrm{C}), 126.9,124.4,123.45(\mathrm{q}, J=274.7 \mathrm{~Hz}), 123.44$ (q, $J=271.7 \mathrm{~Hz}), 123.37$ (q, $J=$ $4.7 \mathrm{~Hz}), 123.1,61.8,59.5,39.3,34.6,13.8$.

${ }^{19}$ F NMR $\left(376 \mathrm{MHz}, \mathrm{CDCl}_{3}, \delta\right):-60.9,-62.8$.

HRMS (ESI): Calculated for $\mathrm{C}_{29} \mathrm{H}_{23} \mathrm{~F}_{6} \mathrm{O}_{3}(\mathrm{M}+\mathrm{H})^{+}$533.1551, found 533.1546.
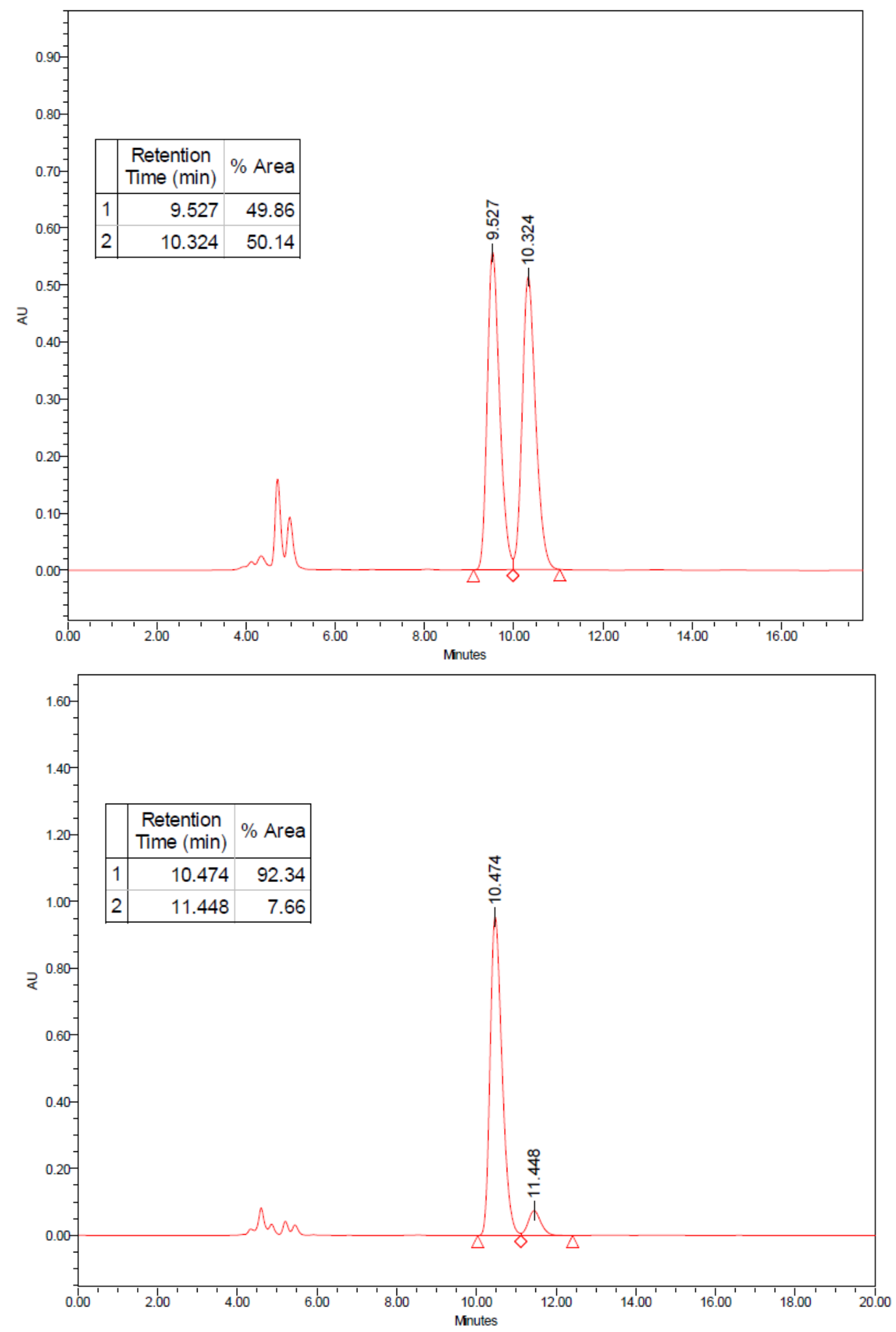
<smiles>COC1(Cc2ccccc2)CC(=Cc2ccc(C(F)(F)F)cc2C(F)(F)F)c2ccccc2C1=O</smiles>

Yellow oil (73 mg, $94 \%$ ) obtained from the general procedure (3) using $\mathbf{S}_{\mathbf{2}}$ (71 $\mathrm{mg}, 0.15 \mathrm{mmol}$ ) and phenylboronic acid (37 $\mathrm{mg}, 0.30 \mathrm{mmol}$ ).

$\mathbf{R}_{\mathbf{f}}=0.33$ (petroleum ether/EtOAc 9:1).

HPLC (Chiralpak IB hexane $/ \mathrm{PrOH}$ 97:3, $0.9 \mathrm{~mL} / \mathrm{min}$ ): $\mathrm{Rt}=9.1 \mathrm{~min}$ (minor), $12.3 \mathrm{~min}$ (major). $[\alpha]_{\mathbf{D}^{20}}=-189\left(\mathrm{c}=2.0, \mathrm{CHCl}_{3}\right)$ for an enantiomeric excess of $84 \%$.

${ }^{1}$ H NMR $\left(400 \mathrm{MHz}, \mathrm{CDCl}_{3}, \delta\right): 8.15(\mathrm{dd}, J=7.9,1.4 \mathrm{~Hz}, 1 \mathrm{H}), 8.01-7.96(\mathrm{~m}, 1 \mathrm{H}), 7.80$ (dd, $J$ $=7.5,1.4 \mathrm{~Hz}, 1 \mathrm{H}), 7.73(\mathrm{dd}, J=7.9,1.1 \mathrm{~Hz}, 1 \mathrm{H}), 7.65-7.57(\mathrm{~m}, 2 \mathrm{H}), 7.52-7.44(\mathrm{~m}, 1 \mathrm{H}), 7.44-$ $7.40(\mathrm{~m}, 1 \mathrm{H}), 7.21-7.07(\mathrm{~m}, 5 \mathrm{H}), 3.59(\mathrm{~s}, 3 \mathrm{H}), 3.50(\mathrm{~d}, J=13.7 \mathrm{~Hz}, 1 \mathrm{H}), 3.31(\mathrm{dd}, J=14.4$, $2.1 \mathrm{~Hz}, 1 \mathrm{H}), 3.18(\mathrm{~d}, J=13.7 \mathrm{~Hz}, 1 \mathrm{H}), 2.68(\mathrm{dd}, J=14.4,2.1 \mathrm{~Hz}, 1 \mathrm{H})$.

${ }^{13}$ C NMR (101 MHz, $\mathrm{CDCl}_{3}, \delta$ ): 193.5, 171.4, 139.8, 139.1, 135.9, 135.4, 134.1, 131.7, 131.1, $130.6(2 \mathrm{C}), 129.8$ (q, $J=33.6 \mathrm{~Hz}), 129.7$ (q, $J=30.8 \mathrm{~Hz}), 129.3,128.43(\mathrm{q}, J=3.7 \mathrm{~Hz})$, 128.38, 128.2 (2C), 126.9, 124.6, 123.45 (hept, $J=5.0 \mathrm{~Hz}$ ), 123.43 (q, $J=274.3 \mathrm{~Hz}$ ), 123.42 $(\mathrm{q}, J=271.6 \mathrm{~Hz}), 123.3,60.4,59.6,52.7,39.5,34.6,21.0,14.2$.

${ }^{19}$ F NMR $\left(376 \mathrm{MHz}, \mathrm{CDCl}_{3}, \delta\right):-60.9,-62.8$.

HRMS (ESI): Calculated for $\mathrm{C}_{28} \mathrm{H}_{21} \mathrm{~F}_{6} \mathrm{O}_{3}(\mathrm{M}+\mathrm{H})^{+} 519.1395$, found 519.1388 .

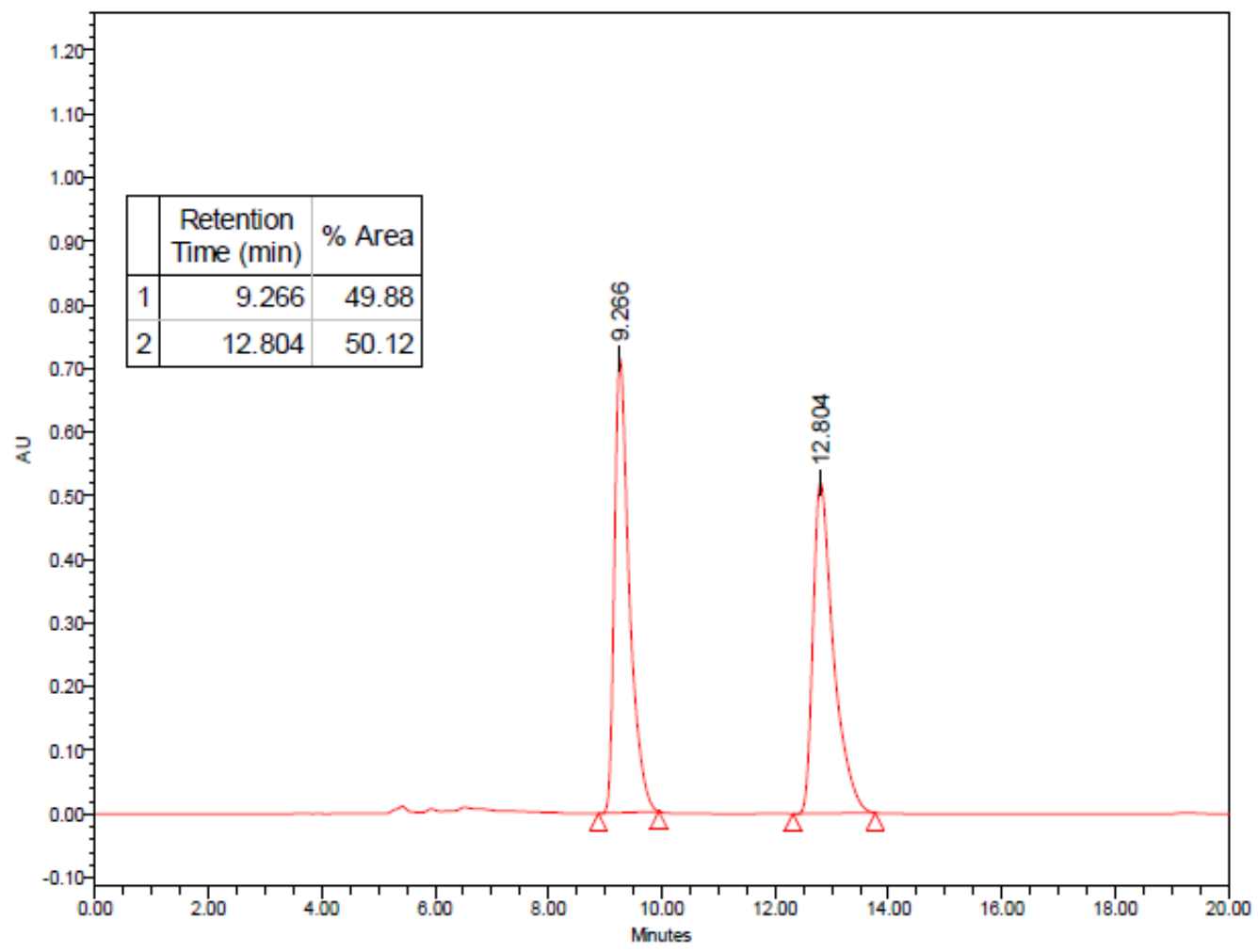




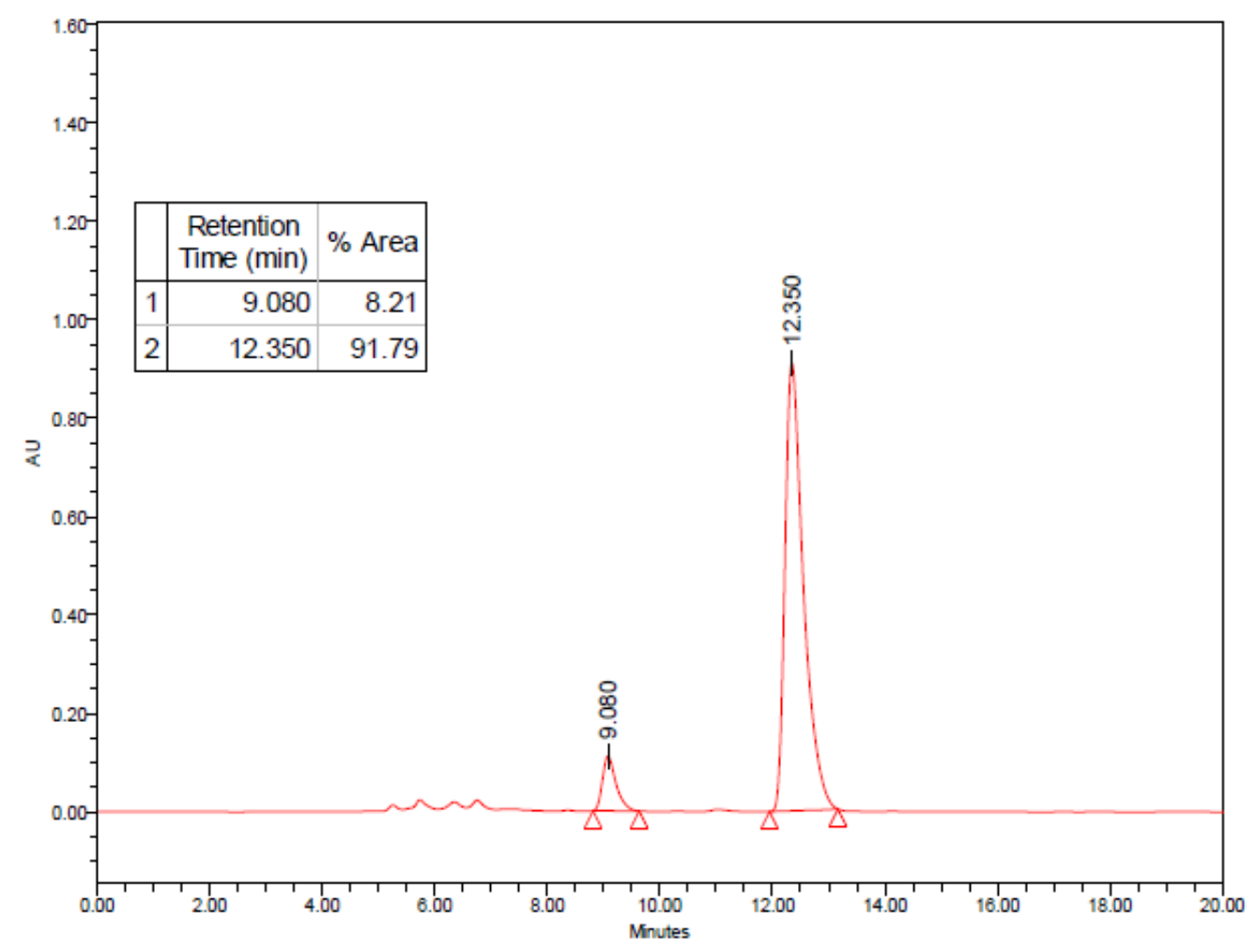

Isopropyl

(R,E)-2-benzyl-4-(2,4-bis(trifluoromethyl)benzylidene)-1-oxo-1,2,3,4tetrahydronaphthalene-2-carboxylate $(2 \mathrm{a}$ '")<smiles>CC(C)OC(=O)C1(Cc2ccc(C(F)(F)F)cc2)CC(=Cc2ccc(C(F)(F)F)cc2)c2ccccc2C1=O</smiles>

Yellow oil (69 mg, $84 \%$ ) obtained from the general procedure (3) using $\mathbf{S}_{\mathbf{3}}$ (79 $\mathrm{mg}, 0.15 \mathrm{mmol}$ ) and phenylboronic acid (37 $\mathrm{mg}, 0.30 \mathrm{mmol})$.

$\mathbf{R}_{\mathbf{f}}=0.44$ (petroleum ether/EtOAc 9:1).

HPLC (Chiralpak IB hexane $/ \mathrm{PrOH} 97: 3,0.5 \mathrm{~mL} / \mathrm{min}$ ): $\mathrm{Rt}=11.0 \mathrm{~min}$ (minor), $12.6 \mathrm{~min}$ (major).

$[\alpha]_{\mathbf{D}}{ }^{\mathbf{2 0}}=-176\left(\mathrm{c}=1.6, \mathrm{CHCl}_{3}\right)$ for an enantiomeric excess of $68 \%$.

${ }^{1} \mathbf{H}$ NMR $\left(400 \mathrm{MHz}, \mathrm{CDCl}_{3}, \delta\right): 8.11(\mathrm{dd}, J=7.9,1.4 \mathrm{~Hz}, 1 \mathrm{H}), 7.97(\mathrm{~s}, 1 \mathrm{H}), 7.79(\mathrm{~d}, J=7.9$ $\mathrm{Hz}, 1 \mathrm{H}), 7.75(\mathrm{~d}, J=7.9 \mathrm{~Hz}, 1 \mathrm{H}), 7.66(\mathrm{~d}, J=7.9 \mathrm{~Hz}, 1 \mathrm{H}), 7.62-7.56(\mathrm{~m}, 1 \mathrm{H}), 7.49-7.40(\mathrm{~m}$, 2H), 7.20-7.11 (m, 5H), 4.89 (hept, $J=6.2 \mathrm{~Hz}, 1 \mathrm{H}), 3.42$ (d, $J=13.8 \mathrm{~Hz}, 1 \mathrm{H}), 3.36$ (d, $J=$ $14.7 \mathrm{~Hz}, 1 \mathrm{H}), 3.18(\mathrm{~d}, J=13.8 \mathrm{~Hz}, 1 \mathrm{H}), 2.62(\mathrm{dd}, J=14.7,2.1 \mathrm{~Hz}, 1 \mathrm{H}), 1.08(\mathrm{~d}, J=6.2 \mathrm{~Hz}$, $3 \mathrm{H}), 0.93(\mathrm{~d}, J=6.2 \mathrm{~Hz}, 3 \mathrm{H})$.

${ }^{13}$ C NMR (101 MHz, $\left.\mathrm{CDCl}_{3}, \delta\right): 193.9,170.4,139.3,139.2,136.1,135.3,133.8,131.7,131.6$, $130.7(2 \mathrm{C}), 129.75$ (q, $J=33.7 \mathrm{~Hz}), 129.66$ (q, $J=30.8 \mathrm{~Hz}), 129.2,128.4(\mathrm{q}, J=3.9 \mathrm{~Hz})$, $128.14,128.08$ (2C), 126.8, 124.2, 123.46 (q, $J=274.5 \mathrm{~Hz}), 123.44$ (q, $J=271.9 \mathrm{~Hz}), 123.3$ (q, $J=4.4 \mathrm{~Hz}), 122.8,69.6,59.4,39.1,34.6,21.4,21.3$.

${ }^{19}$ F NMR (376 MHz, $\left.\mathrm{CDCl}_{3}, \delta\right):-60.9,-62.8$.

HRMS (ESI): Calculated for $\mathrm{C}_{30} \mathrm{H}_{25} \mathrm{~F}_{6} \mathrm{O}_{3}(\mathrm{M}+\mathrm{H})^{+}$547.1708, found 547.1701. 

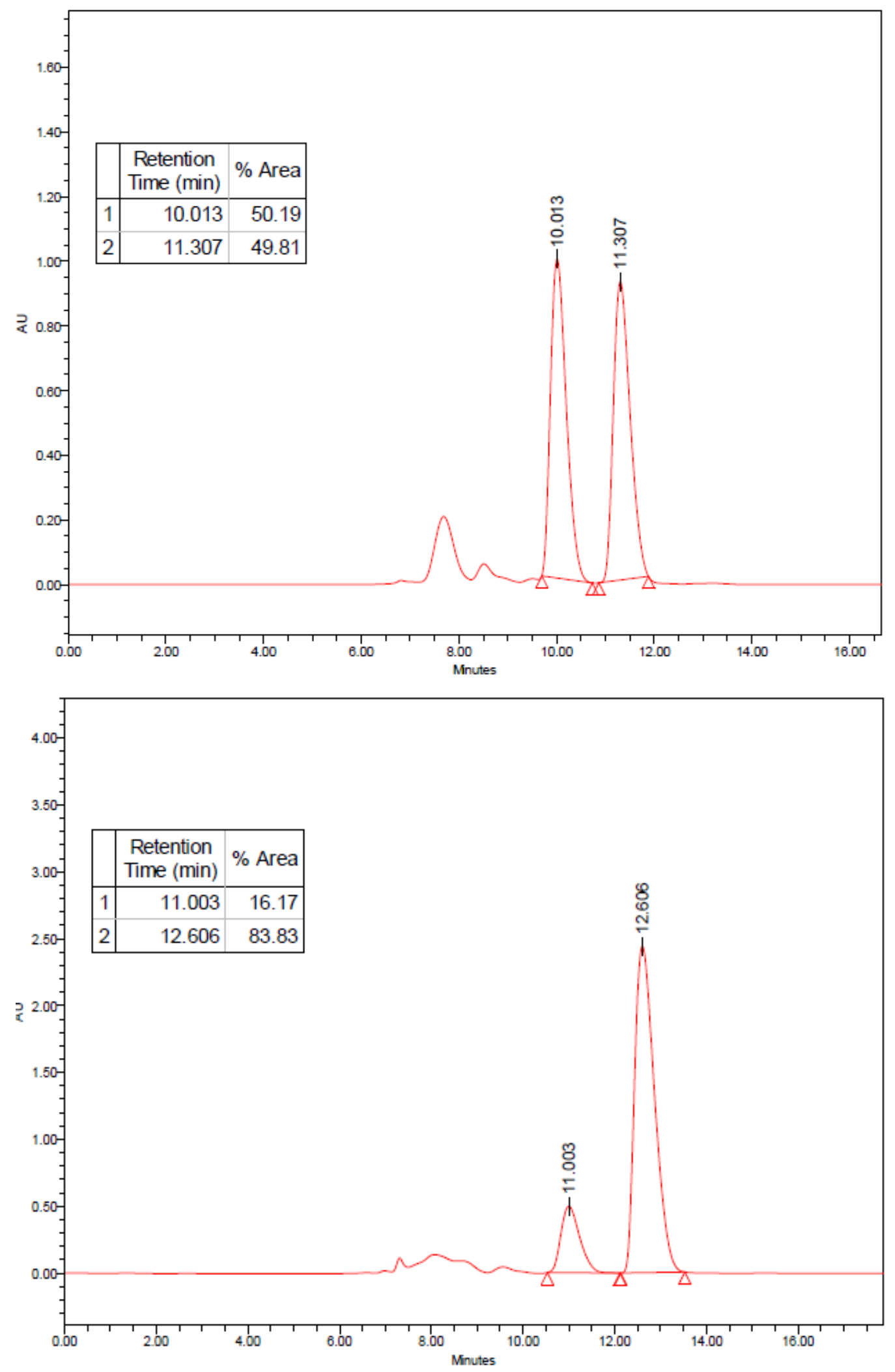
<smiles>COC(=O)C1(C(C)=O)C/C(=C\c2ccc(C(F)(F)F)cc2C(F)(F)F)c2ccccc2C1=O</smiles>

Yellow oil (57 mg, $87 \%$ ) obtained from the general procedure (3) using $\mathbf{S}_{\mathbf{4}}$ (59 $\mathrm{mg}, 0.15 \mathrm{mmol}$ ) and phenylboronic acid (37 $\mathrm{mg}, 0.30 \mathrm{mmol})$.

$\mathbf{R}_{\mathbf{f}}=0.34$ (petroleum ether/EtOAc 9:1).

HPLC (Chiralpak ID hexane $/ \mathrm{PrOH}$ 95:5, $0.5 \mathrm{~mL} / \mathrm{min}$ ): $\mathrm{Rt}=16.7 \mathrm{~min}$ (major), $19.5 \mathrm{~min}$ (minor).

$[\alpha]_{\mathrm{D}}{ }^{\mathbf{2 0}}=+68\left(\mathrm{c}=0.75, \mathrm{CHCl}_{3}\right)$ for an enantiomeric excess of $85 \%$.

${ }^{1}$ H NMR $\left(400 \mathrm{MHz}, \mathrm{CDCl}_{3}, \delta\right): 8.13(\mathrm{dd}, J=7.8,1.4 \mathrm{~Hz}, 1 \mathrm{H}), 7.99(\mathrm{~s}, 1 \mathrm{H}), 7.87(\mathrm{~d}, J=8.1$ $\mathrm{Hz}, 1 \mathrm{H}), 7.76(\mathrm{dd}, J=8.1,1.4 \mathrm{~Hz}, 1 \mathrm{H}), 7.69-7.61(\mathrm{~m}, 1 \mathrm{H}), 7.58(\mathrm{~d}, J=8.1 \mathrm{~Hz}, 1 \mathrm{H}), 7.54-7.47$ (m, 1H), $7.43(\mathrm{~s}, 1 \mathrm{H}), 3.64(\mathrm{~s}, 3 \mathrm{H}), 3.39(\mathrm{dd}, J=14.1,1.4 \mathrm{~Hz}, 1 \mathrm{H}), 2.74(\mathrm{dd}, J=14.1,1.4 \mathrm{~Hz}$, $1 \mathrm{H}), 1.43$ (s, 3H).

${ }^{13}$ C NMR (101 MHz, $\mathrm{CDCl}_{3}, \delta$ ): 195.2, 172.5, 139.9, 139.3, 135.5, 134.2, 131.8, 130.1 (q, $J=$ $33.9 \mathrm{~Hz}), 130.2,129.9(\mathrm{q}, J=31.0 \mathrm{~Hz}), 128.6(\mathrm{q}, J=3.6 \mathrm{~Hz}), 128.3,126.2,124.7,123.8,123.5$ (hept, $J=4.1 \mathrm{~Hz}$ ), 123.4 (q, $J=273.0 \mathrm{~Hz}, 2 \mathrm{C}), 54.9,52.7,37.8,20.4$.

${ }^{19}$ F NMR (376 MHz, $\left.\mathrm{CDCl}_{3}, \delta\right):-60.9,-62.8$.

HRMS (ESI): Calculated for $\mathrm{C}_{22} \mathrm{H}_{17} \mathrm{~F}_{6} \mathrm{O}_{3}(\mathrm{M}+\mathrm{H})^{+} 443.1082$, found 443.1076 .

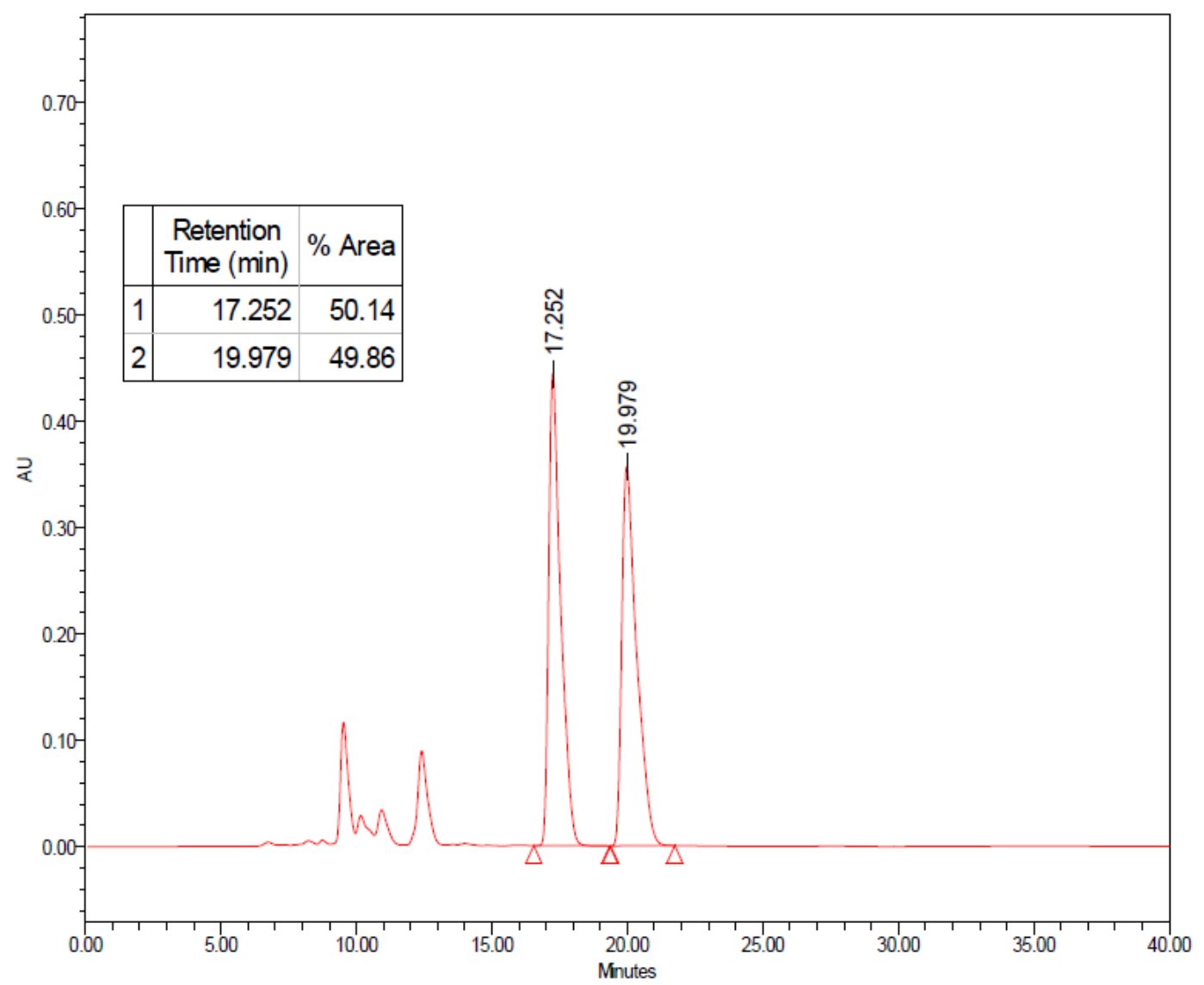




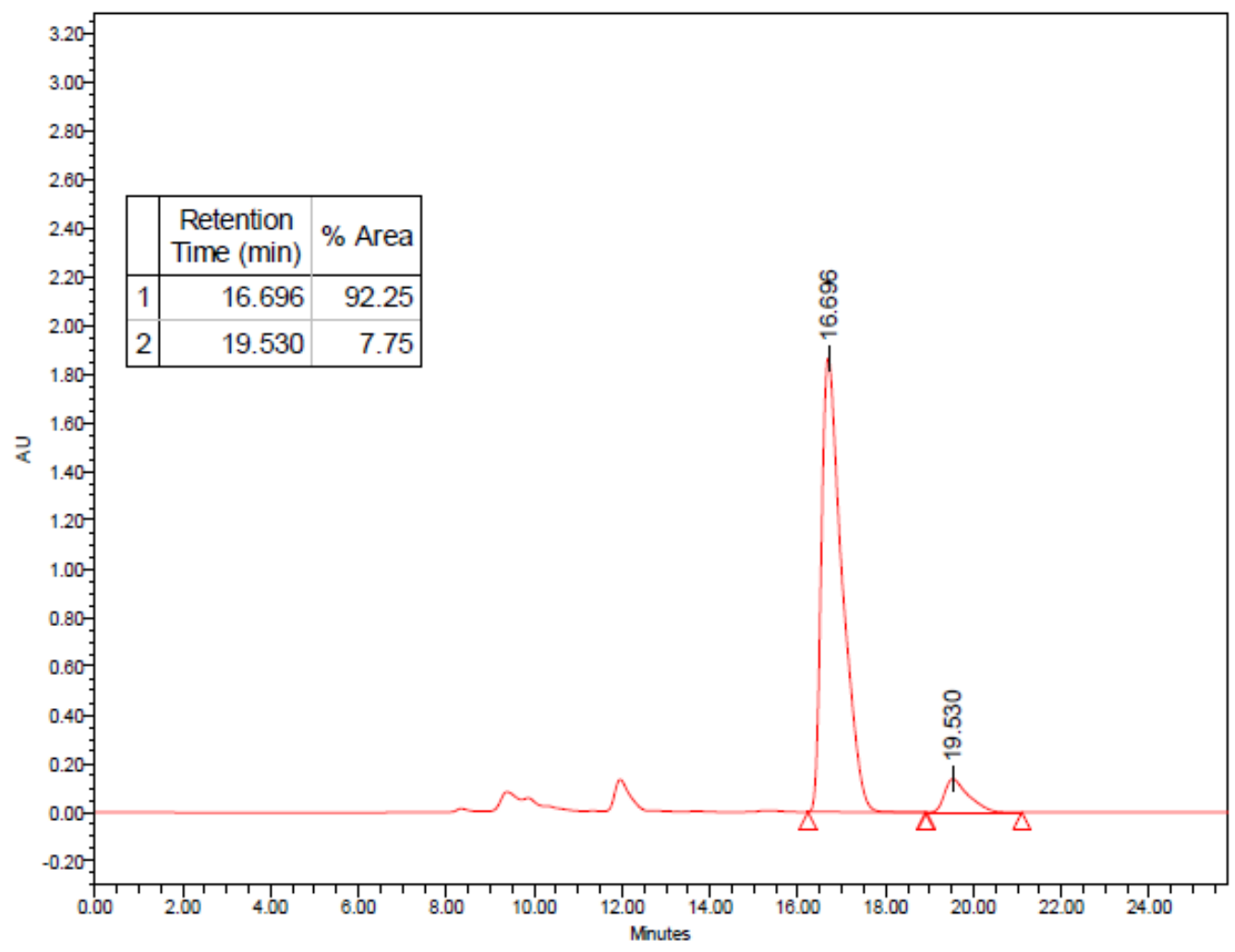

Methyl (R,E)-4-(2,4-bis(trifluoromethyl)benzylidene)-1-oxo-2-phenethyl-1,2,3,4tetrahydronaphthalene-2-carboxylate (2c)<smiles>COC(=O)[C@]1(CCc2ccccc2)C/C(=C\c2ccc(C(F)(F)F)cc2C(F)(F)F)c2ccccc2C1=O</smiles>

Orange oil (75 mg, $94 \%$ ) obtained from the general procedure (3) using $\mathbf{S}_{\mathbf{5}}$ (73 $\mathrm{mg}, 0.15 \mathrm{mmol}$ ) and phenylboronic acid (37 $\mathrm{mg}, 0.30 \mathrm{mmol}$ ).

$\mathbf{R}_{\mathbf{f}}=0.29$ (petroleum ether/EtOAc 9:1).

HPLC (Chiralpak IB hexane $/ \mathrm{iPrOH} 95: 5,0.5 \mathrm{~mL} / \mathrm{min}$ ): $\mathrm{Rt}=14.8 \mathrm{~min}$ (minor), $16.5 \mathrm{~min}$ (major).

$[\boldsymbol{\alpha}]_{\mathbf{D}}{ }^{\mathbf{2 0}}=-81\left(\mathrm{c}=1.45, \mathrm{CHCl}_{3}\right)$ for an enantiomeric excess of $85 \%$.

${ }^{1} \mathbf{H}$ NMR $\left(400 \mathrm{MHz}, \mathrm{CDCl}_{3}, \delta\right): 8.14(\mathrm{ddd}, J=8.0,1.5,0.5 \mathrm{~Hz}, 1 \mathrm{H}), 8.00(\mathrm{~s}, 1 \mathrm{H}), 7.88(\mathrm{~d}, J=$ $8.0 \mathrm{~Hz}, 1 \mathrm{H}), 7.77(\mathrm{dt}, J=8.0,0.5 \mathrm{~Hz}, 1 \mathrm{H}), 7.68-7.62(\mathrm{~m}, 2 \mathrm{H}), 7.55-7.48(\mathrm{~m}, 1 \mathrm{H}), 7.47(\mathrm{~s}, 1 \mathrm{H})$, 7.26-7.10 (m, 5H), 3.64 (s, 3H), 3.42 (dd, $J=14.3,1.6 \mathrm{~Hz}, 1 \mathrm{H}), 2.93$ (dd, $J=14.3,1.6 \mathrm{~Hz}$, $1 \mathrm{H}), 2.71-2.41(\mathrm{~m}, 2 \mathrm{H}), 2.29-2.09(\mathrm{~m}, 2 \mathrm{H})$.

${ }^{13}$ C NMR (101 MHz, $\left.\mathrm{CDCl}_{3}, \delta\right):$ 194.4, 171.6, 141.2, 139.6, 139.1, 135.3, 134.2, 131.7, 130.8, $130.0(\mathrm{q}, J=33.6 \mathrm{~Hz}), 129.8(\mathrm{q}, J=30.8 \mathrm{~Hz}), 128.7(\mathrm{q}, J=3.6 \mathrm{~Hz}), 128.5,128.4(2 \mathrm{C}), 128.3$ (3C), 126.0, 124.6, 123.7, 123.5 (hept, $J=4.7 \mathrm{~Hz}), 123.42$ (q, $J=274.4 \mathrm{~Hz}$ ), 123.37 (q, $J=$ $272.1 \mathrm{~Hz}), 58.6,52.6,35.8,35.4,31.1$.

${ }^{19}$ F NMR $\left(376 \mathrm{MHz}, \mathrm{CDCl}_{3}, \delta\right):-60.9,-62.8$.

HRMS (ESI): Calculated for $\mathrm{C}_{29} \mathrm{H}_{23} \mathrm{~F}_{6} \mathrm{O}_{3}(\mathrm{M}+\mathrm{H})^{+} 533.1551$, found 533.1545. 

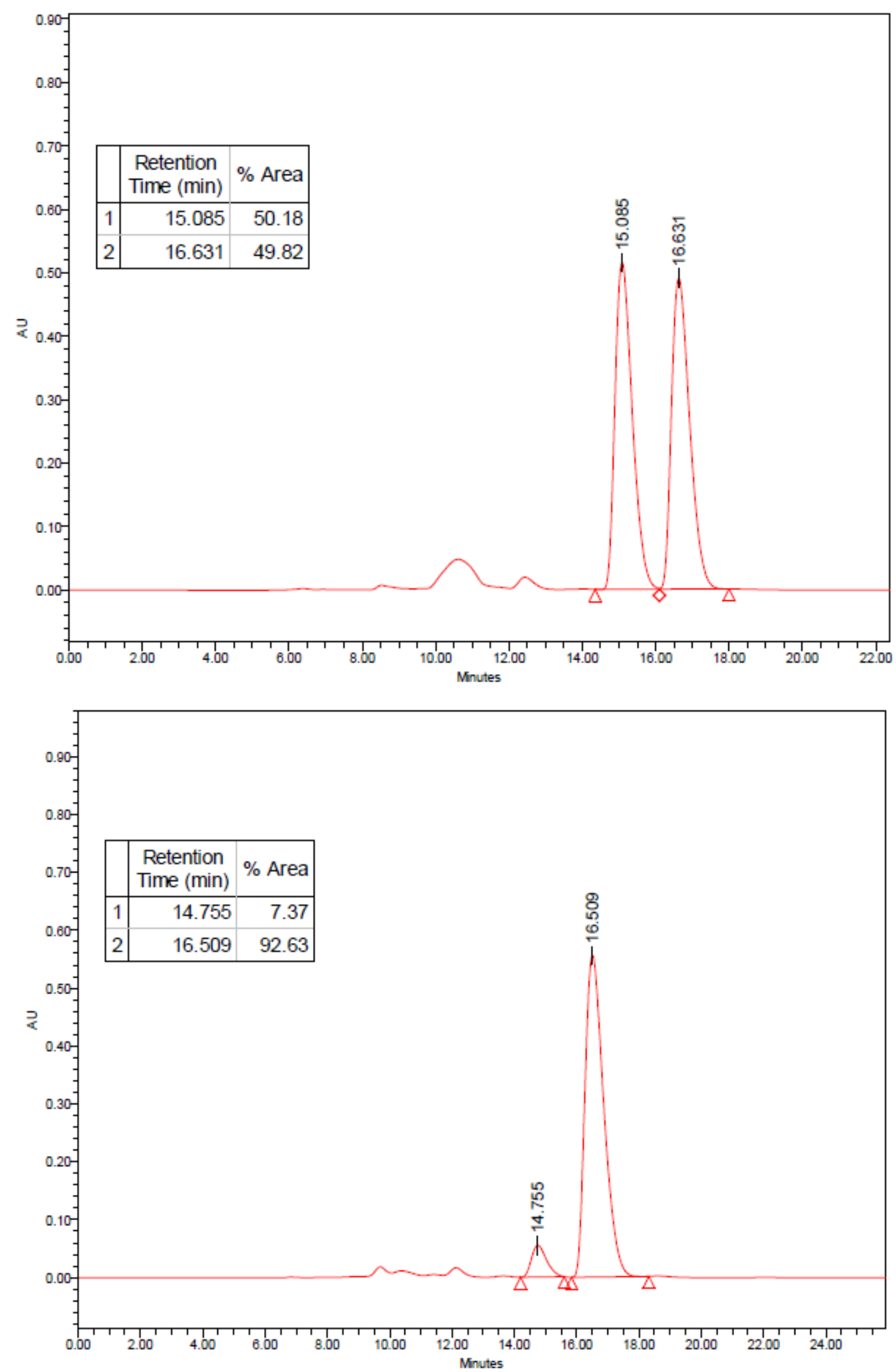
<smiles>COC(=O)[C@]1(Cc2ccccc2)C/C(=C\c2ccc(C(F)(F)F)cc2C(F)(F)F)c2ccccc2C1=O</smiles>

Brown oil (60 mg, $75 \%$ ) obtained from the general procedure (3) using $\mathbf{S}_{\mathbf{9}}$ (73 $\mathrm{mg}, 0.15 \mathrm{mmol}$ ) and phenylboronic acid (37 $\mathrm{mg}, 0.30 \mathrm{mmol})$.

$\mathbf{R}_{\mathbf{f}}=0.34$ (petroleum ether/EtOAc 9:1).

HPLC (Chiralpak ID hexane $/ \mathrm{PrOH}$ 95:5, $0.5 \mathrm{~mL} / \mathrm{min}$ ): $\mathrm{Rt}=13.8 \mathrm{~min}$ (major), $16.2 \mathrm{~min}$ (minor).

$[\boldsymbol{\alpha}]_{\mathbf{D}}{ }^{\mathbf{2 0}}=-74\left(\mathrm{c}=0.7, \mathrm{CHCl}_{3}\right)$ for an enantiomeric excess of $64 \%$.

${ }^{1} \mathbf{H}$ NMR $\left(400 \mathrm{MHz}, \mathrm{CDCl}_{3}, \delta\right): 8.08(\mathrm{dd}, J=7.8,1.4 \mathrm{~Hz}, 1 \mathrm{H}), 7.95(\mathrm{~s}, 1 \mathrm{H}), 7.86-7.73(\mathrm{~m}, 2 \mathrm{H})$, 7.68 (dd, $J=7.3,1.4 \mathrm{~Hz}, 1 \mathrm{H}), 7.66-7.58(\mathrm{~m}, 1 \mathrm{H}), 7.54-7.40(\mathrm{~m}, 2 \mathrm{H}), 7.35-7.18(\mathrm{~m}, 5 \mathrm{H}), 4.90$ $(\mathrm{d}, J=11.1 \mathrm{~Hz}, 1 \mathrm{H}), 4.56(\mathrm{~d}, J=11.1 \mathrm{~Hz}, 1 \mathrm{H}), 3.77(\mathrm{~s}, 3 \mathrm{H}), 3.33(\mathrm{dd}, J=14.6,1.7 \mathrm{~Hz}, 1 \mathrm{H})$, $3.25(\mathrm{dd}, J=14.6,1.0 \mathrm{~Hz}, 1 \mathrm{H})$.

${ }^{13}$ C NMR (101 MHz, $\left.\mathrm{CDCl}_{3}, \delta\right): 191.8,170.1,140.2,139.1,137.6,134.7,133.9,132.2$ (2C), $129.8(\mathrm{q}, J=33.6 \mathrm{~Hz}), 129.7(\mathrm{q}, J=30.8 \mathrm{~Hz}), 129.4,128.6(\mathrm{q}, J=3.7 \mathrm{~Hz}), 128.3,128.2(2 \mathrm{C})$, 127.8 (2C), 127.7, 124.6, 124.4, 123.43 (q, $J=274.3 \mathrm{~Hz}), 123.42$ (q, $J=271.6 \mathrm{~Hz}), 123.2$ (hept, $J=5.0 \mathrm{~Hz}), 83.1,69.5,52.6,37.6$.

${ }^{19}$ F NMR $\left(376 \mathrm{MHz}, \mathrm{CDCl}_{3}, \delta\right):-61.08,-62.84$.

HRMS (ESI): Calculated for $\mathrm{C}_{28} \mathrm{H}_{20} \mathrm{~F}_{6} \mathrm{O}_{4} \mathrm{Na}(\mathrm{M}+\mathrm{Na})^{+} 557.1163$, found 557.1158.

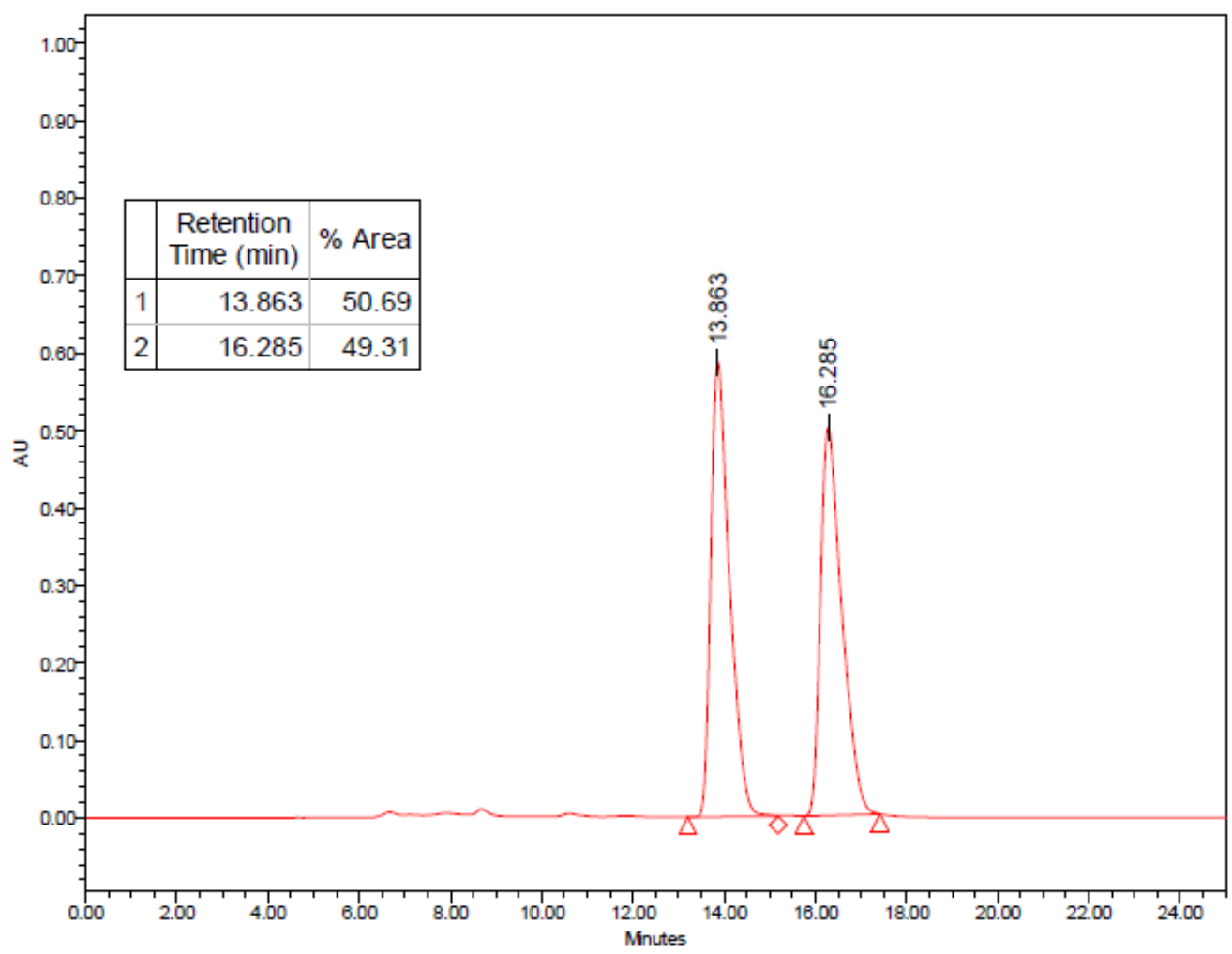




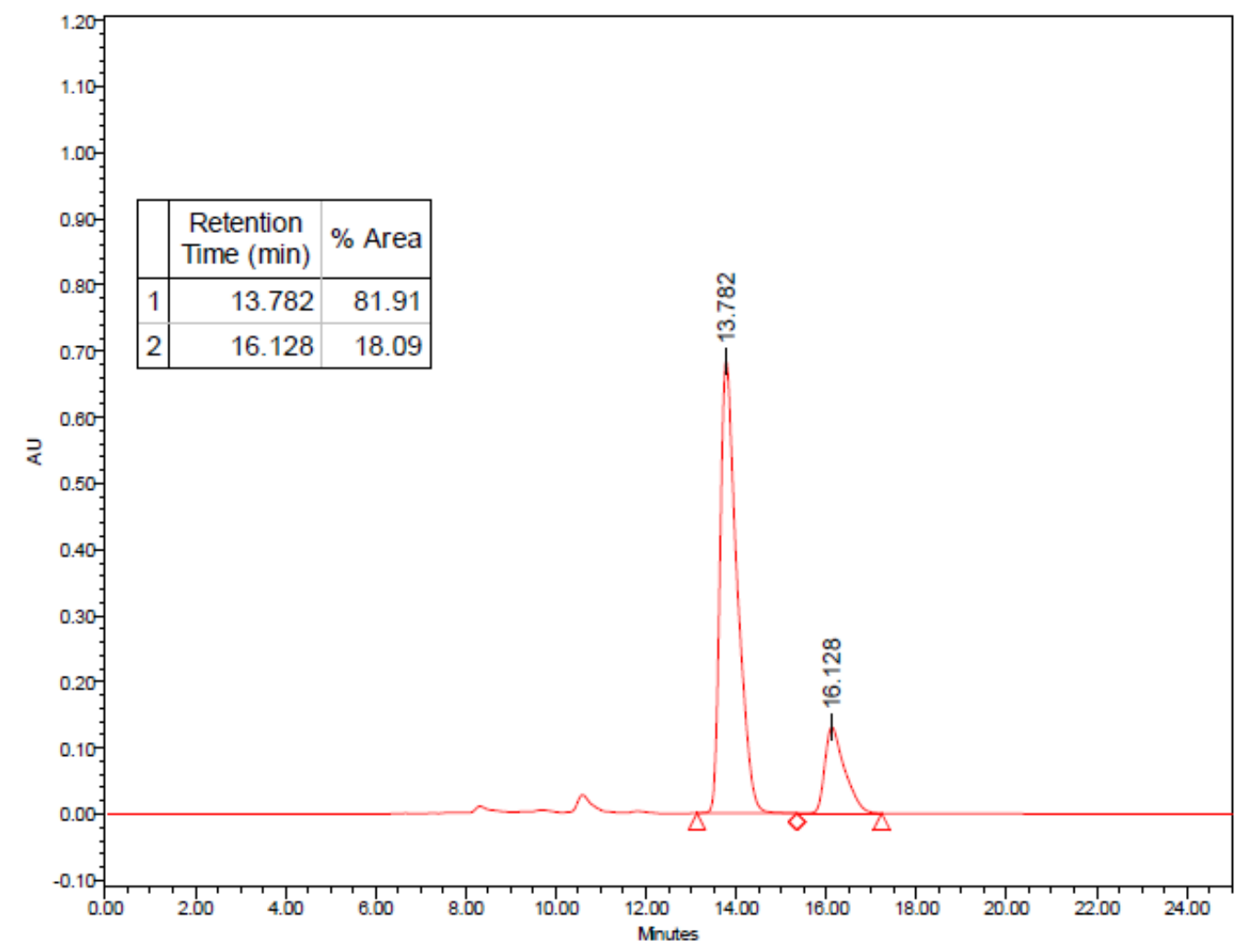

Ethyl (R,E)-2-benzyl-4-(2,4-bis(trifluoromethyl)benzylidene)-7-methoxy-1-oxo-1,2,3,4tetrahydronaphthalene-2-carboxylate (2e)<smiles>CCOC(=O)C1(Cc2ccccc2)CC(=Cc2ccc(C(F)(F)F)cc2C(F)(F)F)c2ccc(OC)cc2C1=O</smiles>

Orange oil (77 mg, $92 \%$ ) obtained from the general procedure (3) using $\mathbf{S}_{\mathbf{1}}$ ( $75 \mathrm{mg}, 0.15 \mathrm{mmol}$ ) and 4-methoxyphenylboronic acid (46 $\mathrm{mg}, 0.30 \mathrm{mmol})$.

$\mathbf{R}_{\mathbf{f}}=0.28$ (petroleum ether/EtOAc 9:1).

HPLC (Chiralpak IB hexane/ $i \operatorname{PrOH} 95: 5,1.0 \mathrm{~mL} / \mathrm{min}$ ): $\mathrm{Rt}=6.2 \mathrm{~min}$ (minor), $7.5 \mathrm{~min}$ (major). $[\alpha]_{\mathbf{D}}{ }^{\mathbf{2 0}}=-244\left(\mathrm{c}=1.8, \mathrm{CHCl}_{3}\right)$ for an enantiomeric excess of $85 \%$.

${ }^{1} \mathbf{H}$ NMR $\left(400 \mathrm{MHz}, \mathrm{CDCl}_{3}, \delta\right): 7.96(\mathrm{~s}, 1 \mathrm{H}), 7.78(\mathrm{~d}, J=8.2 \mathrm{~Hz}, 1 \mathrm{H}), 7.66(\mathrm{~d}, J=8.8 \mathrm{~Hz}, 1 \mathrm{H})$, $7.60(\mathrm{~d}, J=8.2 \mathrm{~Hz}, 1 \mathrm{H}), 7.58(\mathrm{~d}, J=2.8 \mathrm{~Hz}, 1 \mathrm{H}), 7.29(\mathrm{~s}, 1 \mathrm{H}), 7.21-7.10(\mathrm{~m}, 6 \mathrm{H}), 4.09-3.98$ (m, 2H), $3.90(\mathrm{~s}, 3 \mathrm{H}), 3.45(\mathrm{~d}, J=13.7 \mathrm{~Hz}, 1 \mathrm{H}), 3.34(\mathrm{~d}, J=14.5 \mathrm{~Hz}, 1 \mathrm{H}), 3.17$ (d, $J=13.7$ $\mathrm{Hz}, 1 \mathrm{H}), 2.63(\mathrm{dd}, J=14.5,1.6 \mathrm{~Hz}, 1 \mathrm{H}), 1.05(\mathrm{t}, J=7.1 \mathrm{~Hz}, 3 \mathrm{H})$.

${ }^{13}$ C NMR (101 MHz, $\left.\mathrm{CDCl}_{3}, \delta\right): 193.7,170.9,160.4,139.5,136.0,135.0,132.7,132.5,131.7$, $130.7(2 \mathrm{C}), 129.6(\mathrm{q}, J=30.5 \mathrm{~Hz}), 129.5(\mathrm{q}, J=33.6 \mathrm{~Hz}), 128.4(\mathrm{q}, J=3.5 \mathrm{~Hz}), 128.1(2 \mathrm{C})$, $126.8,126.1,123.50(\mathrm{q}, J=271.7 \mathrm{~Hz}), 123.48(\mathrm{q}, J=274.9 \mathrm{~Hz}), 123.3(\mathrm{q}, J=4.6 \mathrm{~Hz}), 122.5$, $121.3,109.8,61.8,59.5,55.6,39.4,34.7,13.8$.

${ }^{19}$ F NMR $\left(376 \mathrm{MHz}, \mathrm{CDCl}_{3}, \delta\right):-61.1,-62.8$.

HRMS (ESI): Calculated for $\mathrm{C}_{30} \mathrm{H}_{25} \mathrm{~F}_{6} \mathrm{O}_{4}(\mathrm{M}+\mathrm{H})^{+} 563.1657$, found 563.1651 . 

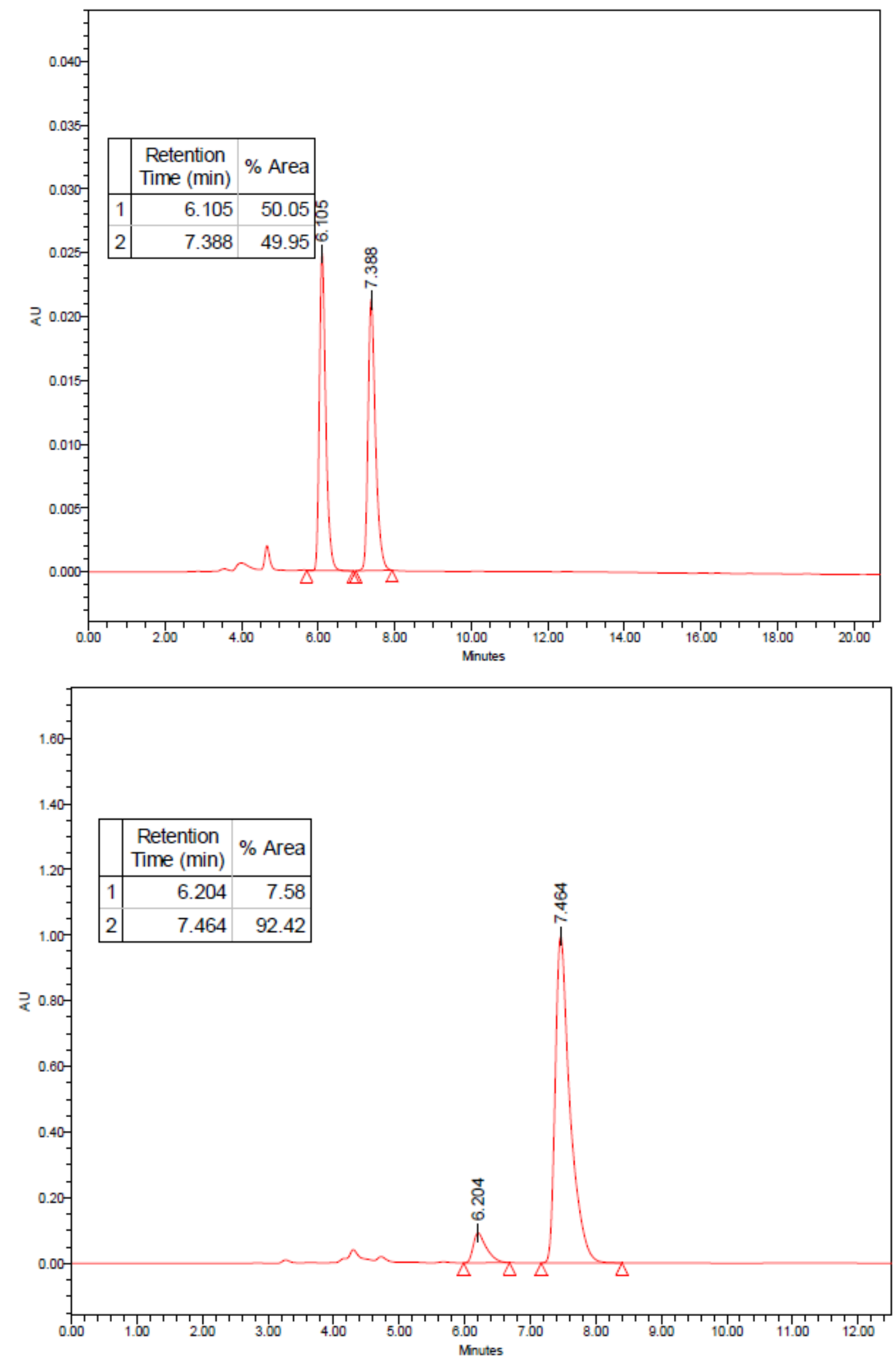
Ethyl (R,E)-6-acetamido-2-benzyl-4-(2,4-bis(trifluoromethyl)benzylidene)-1-oxo-1,2,3,4tetrahydronaphthalene-2-carboxylate (2f)<smiles>CCOC(=O)C1(Cc2ccccc2)CC(=Cc2ccc(C(F)(F)F)cc2C(F)(F)F)c2cc(NC(C)C)ccc2C1=O</smiles>

Brown oil (78 mg, $88 \%$ ) obtained from the general procedure (3) using $\mathbf{S}_{\mathbf{1}}$ (75 $\mathrm{mg}, 0.15 \mathrm{mmol}$ ) and 3-acetamidophenylboronic acid (54 $\mathrm{mg}, 0.30 \mathrm{mmol})$.

Brown oil (636 mg, $90 \%$ ) obtained from the general procedure (3) using $\mathbf{S}_{\mathbf{1}}$ (600 mg, $1.2 \mathrm{mmol}$ ) and 3-acetamidophenylboronic acid (432 $\mathrm{mg}, 2.4 \mathrm{mmol})$.

$\mathbf{R}_{\mathbf{f}}=0.26$ (petroleum ether/EtOAc 6:4).

HPLC (Chiralpak IB hexane $/ \mathrm{iPrOH}$ 90:10, $1.0 \mathrm{~mL} / \mathrm{min}$ ): Rt $=9.9 \mathrm{~min}$ (major), $15.3 \mathrm{~min}$ (minor).

$[\boldsymbol{\alpha}]_{\mathbf{D}}{ }^{\mathbf{2 0}}=-212\left(\mathrm{c}=1.9, \mathrm{CHCl}_{3}\right)$ for an enantiomeric excess of $90 \%$.

${ }^{1}$ H NMR $\left(400 \mathrm{MHz}, \mathrm{CDCl}_{3}, \delta\right): 8.08(\mathrm{~d}, J=8.6 \mathrm{~Hz}, 1 \mathrm{H}), 8.01(\mathrm{~s}, 1 \mathrm{H}), 7.96(\mathrm{~s}, 1 \mathrm{H}), 7.78(\mathrm{dd}$, $J=8.0,1.7 \mathrm{~Hz}, 1 \mathrm{H}), 7.63(\mathrm{~s}, 1 \mathrm{H}), 7.57(\mathrm{~d}, J=8.0 \mathrm{~Hz}, 1 \mathrm{H}), 7.49(\mathrm{dd}, J=8.6,1.7 \mathrm{~Hz}, 1 \mathrm{H}), 7.38$ (s, 1H), $7.20-7.04(\mathrm{~m}, 5 \mathrm{H}), 4.03$ (hept, $J=7.1 \mathrm{~Hz}, 2 \mathrm{H}), 3.46(\mathrm{~d}, J=13.7 \mathrm{~Hz}, 1 \mathrm{H}), 3.29(\mathrm{dd}, J$ $=14.5,2.0 \mathrm{~Hz}, 1 \mathrm{H}), 3.14(\mathrm{~d}, J=13.7 \mathrm{~Hz}, 1 \mathrm{H}), 2.64(\mathrm{dd}, J=14.5,2.0 \mathrm{~Hz}, 1 \mathrm{H}), 2.21(\mathrm{~s}, 3 \mathrm{H})$, $1.04(\mathrm{t}, J=7.1 \mathrm{~Hz}, 3 \mathrm{H})$.

${ }^{13}$ C NMR (101 MHz, $\left.\mathrm{CDCl}_{3}, \delta\right): 192.5,171.0,168.7,143.1,141.2,139.0,136.0,135.4,131.6$, $130.7(2 \mathrm{C}), 129.82(\mathrm{q}, J=33.4 \mathrm{~Hz}), 129.78,129.7$ (q, $J=31.6 \mathrm{~Hz}), 128.4(\mathrm{q}, J=3.4 \mathrm{~Hz})$, 128.1 (2C), 127.2, 126.8, 123.7, 123.4 (q, $J=272.2 \mathrm{~Hz}, 2 \mathrm{C}$ ), 123.3 (hept, $J=4.9 \mathrm{~Hz}$ ), 119.9, 114.0, 61.8, 59.4, 39.4, 34.6, 24.8, 13.8 .

${ }^{19}$ F NMR $\left(376 \mathrm{MHz}, \mathrm{CDCl}_{3}, \delta\right):-60.8,-62.8$.

HRMS (ESI): Calculated for $\mathrm{C}_{31} \mathrm{H}_{26} \mathrm{~F}_{6} \mathrm{NO}_{4}(\mathrm{M}+\mathrm{H})^{+}$590.1766, found 590.1762 . 

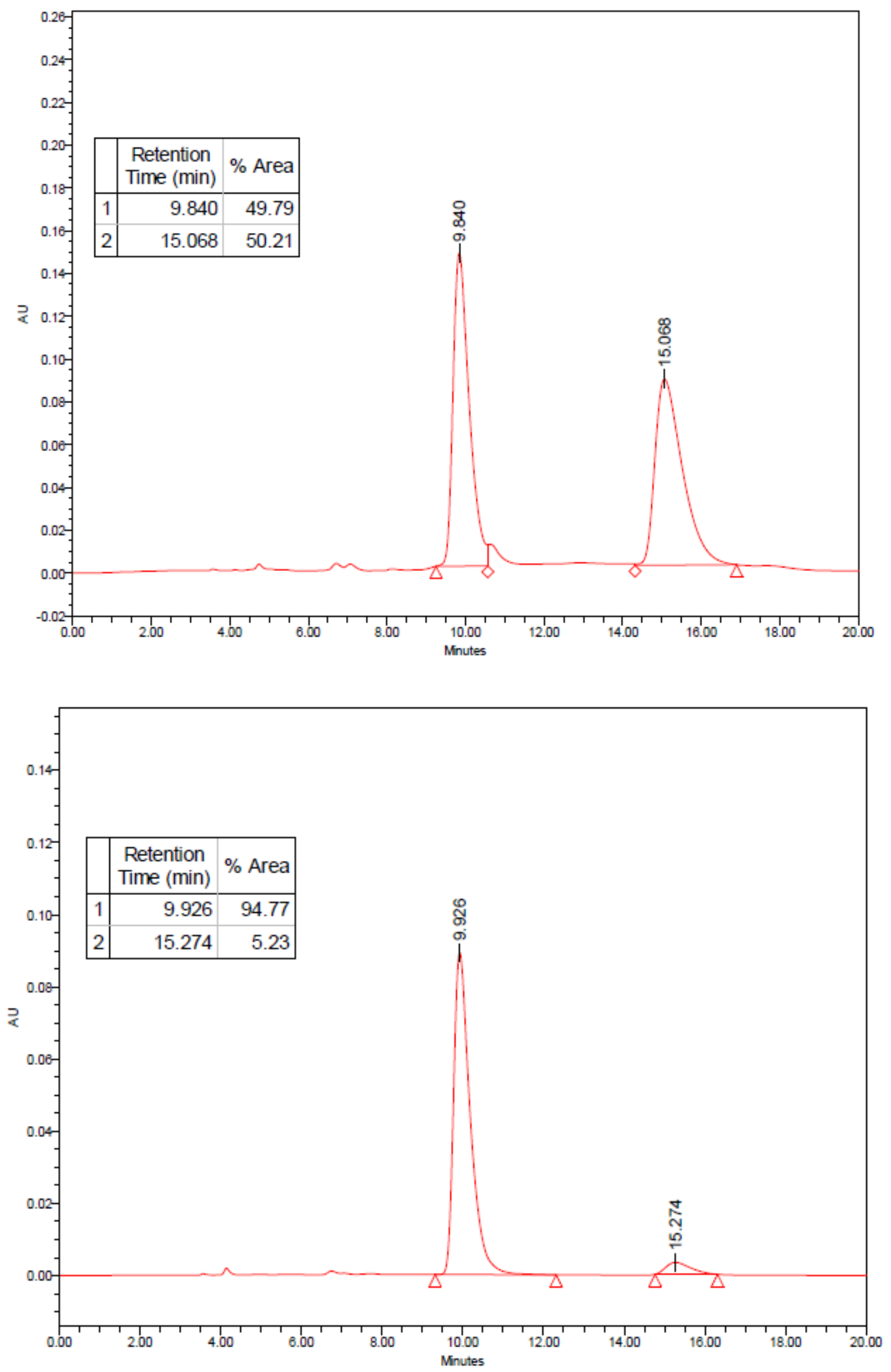
Ethyl (R,E)-2-benzyl-4-(2,4-bis(trifluoromethyl)benzylidene)-7-(tert-butyl)-1-oxo-1,2,3,4tetrahydronaphthalene-2-carboxylate $(2 \mathrm{~g})$<smiles>CCOC(Cc1ccccc1)(Cc1ccc(C(C)(C)C)cc1C)Cc1ccc(C(F)(F)F)cc1C(F)(F)F</smiles>

Orange oil (78 mg, $89 \%$ ) obtained from the general procedure (3) using $\mathbf{S}_{\mathbf{1}}$ (75 $\mathrm{mg}, 0.15 \mathrm{mmol}$ ) and 4-tert-butylphenylboronic acid (54 $\mathrm{mg}, 0.30 \mathrm{mmol})$.

$\mathbf{R}_{\mathbf{f}}=0.52$ (petroleum ether/EtOAc 9:1).

HPLC (Chiralpak ID hexane/ $i \mathrm{PrOH}$ 97:3, $0.3 \mathrm{~mL} / \mathrm{min}$ ): $\mathrm{Rt}=21.8 \mathrm{~min}$ (major), $6.4 \mathrm{~min}$ (minor). $[\boldsymbol{\alpha}]_{\mathbf{D}}{ }^{\mathbf{2 0}}=-154\left(\mathrm{c}=3.0, \mathrm{CHCl}_{3}\right)$ for an enantiomeric excess of $87 \%$.

${ }^{1} \mathbf{H}$ NMR $\left(400 \mathrm{MHz}, \mathrm{CDCl}_{3}, \delta\right): 8.16(\mathrm{~d}, J=2.0 \mathrm{~Hz}, 1 \mathrm{H}), 7.99(\mathrm{~s}, 1 \mathrm{H}), 7.83-7.76(\mathrm{~m}, 1 \mathrm{H}), 7.71$ $(\mathrm{d}, J=8.4 \mathrm{~Hz}, 1 \mathrm{H}), 7.67(\mathrm{dd}, J=8.4,2.1 \mathrm{~Hz}, 1 \mathrm{H}), 7.63(\mathrm{~d}, J=8.1 \mathrm{~Hz}, 1 \mathrm{H}), 7.42(\mathrm{~s}, 1 \mathrm{H}), 7.22$ 7.08 (m, 5H), 4.14-4.00 (m, 2H), 3.45 (d, $J=13.8 \mathrm{~Hz}, 1 \mathrm{H}), 3.38$ (dd, 1H), 3.21 (d, $J=13.8$ $\mathrm{Hz}, 1 \mathrm{H}), 2.68(\mathrm{dd}, J=14.6,2.0 \mathrm{~Hz}, 1 \mathrm{H}), 1.38(\mathrm{~s}, 9 \mathrm{H}), 1.06(\mathrm{t}, J=7.1 \mathrm{~Hz}, 3 \mathrm{H})$.

${ }^{13}$ C NMR (101 MHz, $\left.\mathrm{CDCl}_{3}, \delta\right):$ 194.0, 171.0, 152.7, 139.4, 137.0, 136.1, 135.4, 131.7, 131.6, $130.9,130.7(2 \mathrm{C}), 129.64(\mathrm{q}, J=33.1 \mathrm{~Hz}), 129.63(\mathrm{q}, J=31.5 \mathrm{~Hz}), 128.4(\mathrm{q}, J=3.4 \mathrm{~Hz})$, 128.1 (2C), 126.8, 124.6, 124.3, 123.48 (q, $J=275.0 \mathrm{~Hz}), 123.46$ (q, $J=271.3 \mathrm{~Hz}), 123.30$ (hept, $J=5.0 \mathrm{~Hz}$ ), 122.2, 61.7, 59.6, 39.4, 34.9, 34.5, 31.1 (3C), 13.8.

${ }^{19}$ F NMR (376 MHz, $\left.\mathrm{CDCl}_{3}, \delta\right):-61.0,-62.8$.

HRMS (ESI): Calculated for $\mathrm{C}_{33} \mathrm{H}_{31} \mathrm{~F}_{6} \mathrm{O}_{3}(\mathrm{M}+\mathrm{H})^{+}$589.2177, found 589.2173.

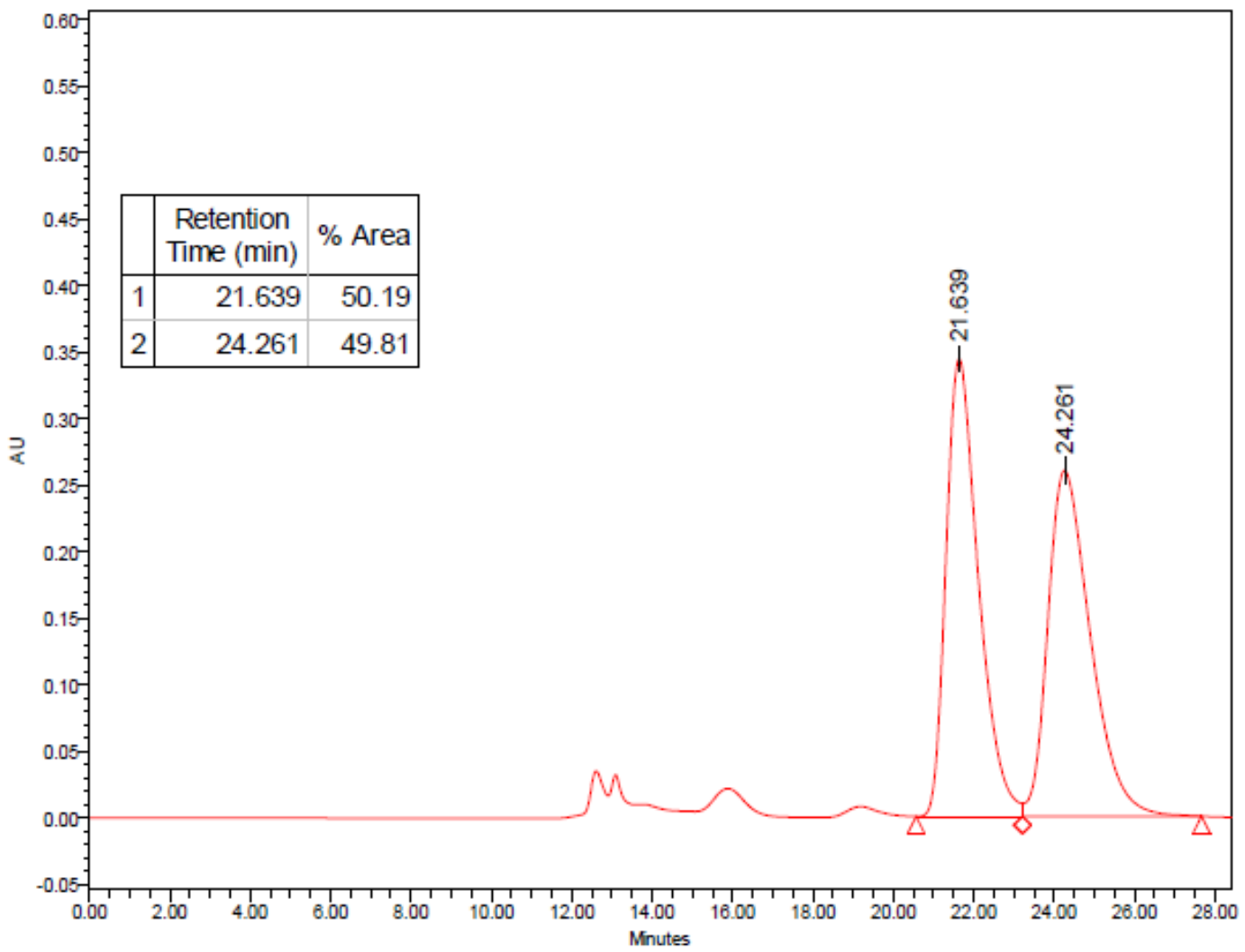




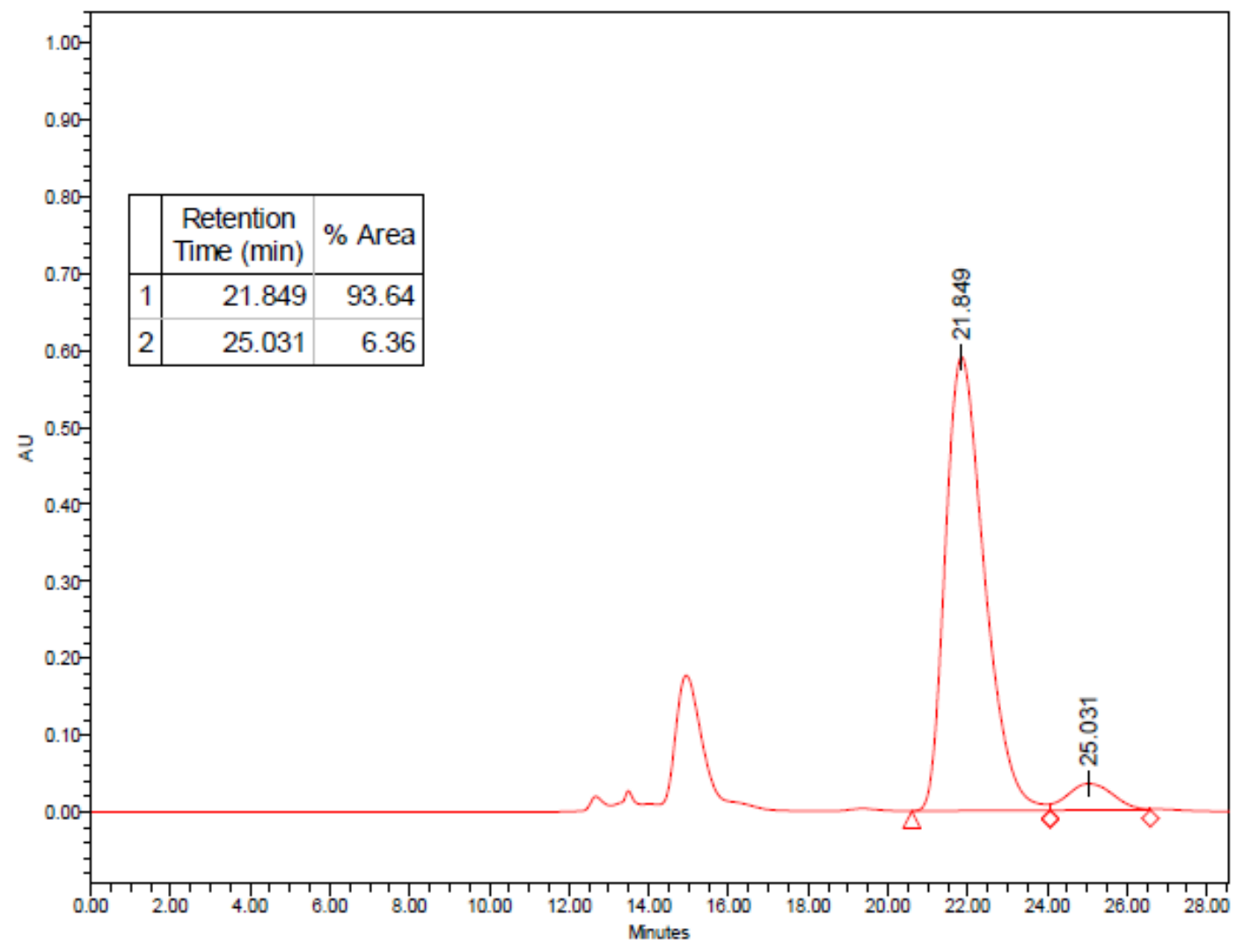

Ethyl

(R,E)-4-(2,4-bis(trifluoromethyl)benzylidene)-2-butyl-1-oxo-1,2,3,4tetrahydronaphthalene-2-carboxylate $(2 \mathrm{~h})$<smiles>CCC[C@@]1(C(=O)OCC)C/C(=C\c2ccc(C(F)(F)F)cc2C(F)(F)F)c2ccccc2C1=O</smiles>

Orange oil (65 mg, $87 \%$ ) obtained from the general procedure (3) using $\mathbf{S}_{\mathbf{6}}$ (70 $\mathrm{mg}, 0.15 \mathrm{mmol}$ ) and phenylboronic acid (37 $\mathrm{mg}, 0.30 \mathrm{mmol})$.

$\mathbf{R}_{\mathbf{f}}=0.42$ (petroleum ether/EtOAc 9:1).

HPLC (Chiralpak ID hexane $/ \mathrm{PrOH} 95: 5,0.5 \mathrm{~mL} / \mathrm{min}$ ): Rt = $13.5 \mathrm{~min}$ (major), $16.4 \mathrm{~min}$ (minor).

$[\boldsymbol{\alpha}]_{\mathbf{D}}{ }^{\mathbf{2 0}}=+97\left(\mathrm{c}=1.2, \mathrm{CHCl}_{3}\right)$ for an enantiomeric excess of $84 \%$.

${ }^{1} \mathbf{H}$ NMR $\left(400 \mathrm{MHz}, \mathrm{CDCl}_{3}, \delta\right): 8.11(\mathrm{dd}, J=8.1,1.4 \mathrm{~Hz}, 1 \mathrm{H}), 7.99(\mathrm{~s}, 1 \mathrm{H}), 7.88(\mathrm{~d}, J=8.1$ $\mathrm{Hz}, 1 \mathrm{H}), 7.76(\mathrm{dd}, J=8.1,1.4 \mathrm{~Hz}, 1 \mathrm{H}), 7.68(\mathrm{~d}, J=8.1 \mathrm{~Hz}, 1 \mathrm{H}), 7.66-7.58(\mathrm{~m}, 1 \mathrm{H}), 7.52-7.46$ $(\mathrm{m}, 1 \mathrm{H}), 7.44(\mathrm{~s}, 1 \mathrm{H}), 4.11(\mathrm{q}, J=7.1 \mathrm{~Hz}, 2 \mathrm{H}), 3.38(\mathrm{dd}, J=14.3,1.4 \mathrm{~Hz}, 1 \mathrm{H}), 2.87(\mathrm{dd}, J=$ $14.3,1.4 \mathrm{~Hz}, 1 \mathrm{H}), 1.95-1.72(\mathrm{~m}, 2 \mathrm{H}), 1.33-1.18(\mathrm{~m}, 2 \mathrm{H}), 1.13(\mathrm{t}, J=7.1 \mathrm{~Hz}, 3 \mathrm{H}), 1.10-1.01$ $(\mathrm{m}, 2 \mathrm{H}), 0.80(\mathrm{t}, J=7.1 \mathrm{~Hz}, 3 \mathrm{H})$.

${ }^{13} \mathbf{C}$ NMR (101 MHz, $\left.\mathrm{CDCl}_{3}, \delta\right): 194.8,171.3,139.7,139.2,135.7,134.0,131.8,130.8,130.0$ $(\mathrm{q}, J=33.7 \mathrm{~Hz}), 129.8(\mathrm{q}, J=30.8 \mathrm{~Hz}), 129.3,128.6(\mathrm{q}, J=4.1 \mathrm{~Hz}), 128.2,124.5,123.44(\mathrm{q}$, $J=274.4 \mathrm{~Hz}), 123.43$ (q, $J=272.0 \mathrm{~Hz}), 123.4(2 \mathrm{C}), 61.5,58.7,34.8,33.3,26.8,22.9,13.9$, 13.7 .

${ }^{19}$ F NMR (376 MHz, $\left.\mathrm{CDCl}_{3}, \delta\right):-61.1,-62.8$. 
HRMS (ESI): Calculated for $\mathrm{C}_{26} \mathrm{H}_{25} \mathrm{~F}_{6} \mathrm{O}_{3}(\mathrm{M}+\mathrm{H})^{+} 499.1708$, found 499.1702.
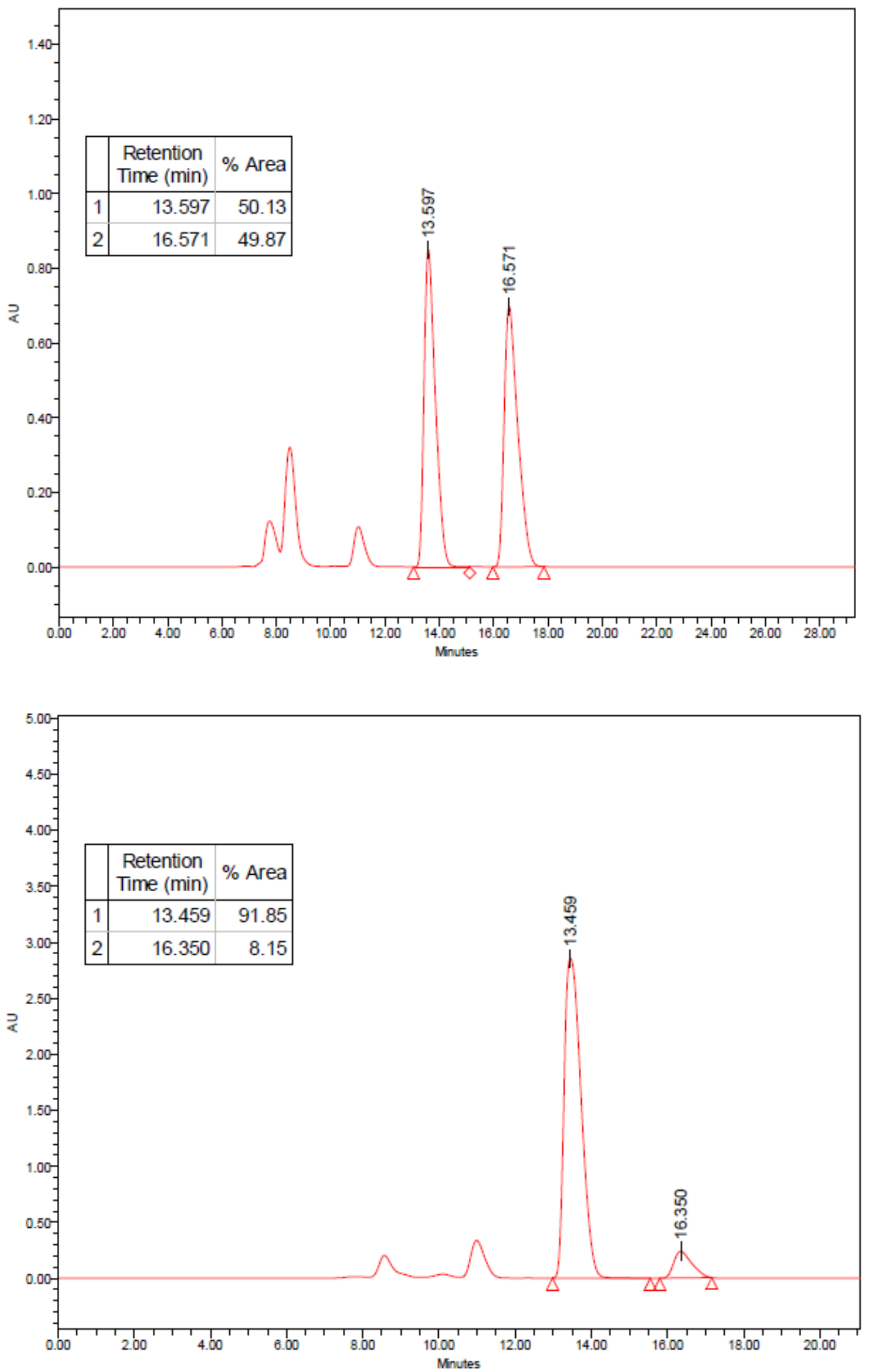
<smiles>CCC[C@@]1(C(=O)OCC)CC(=Cc2ccc(C(F)(F)F)cc2C(F)(F)F)c2cc(C)ccc2C1=O</smiles>

Colorless oil (69 mg, $90 \%$ ) obtained from the general procedure (3) using $\mathbf{S}_{\mathbf{6}}$ (70 $\mathrm{mg}, 0.15$ $\mathrm{mmol}$ ) and 3-methylphenylboronic acid (41 $\mathrm{mg}, 0.30 \mathrm{mmol})$.

$\mathbf{R}_{\mathbf{f}}=0.45$ (petroleum ether/EtOAc 9:1).

HPLC (Chiralpak ID hexane $/ \mathrm{iPrOH}$ 95:5, $0.5 \mathrm{~mL} / \mathrm{min}$ ): $\mathrm{Rt}=14.6 \mathrm{~min}$ (major), $27.7 \mathrm{~min}$ (minor).

$[\boldsymbol{\alpha}]_{\mathbf{D}} \mathbf{2 0}^{\mathbf{2 0}}=+85\left(\mathrm{c}=1.4, \mathrm{CHCl}_{3}\right)$ for an enantiomeric excess of $89 \%$.

${ }^{1}$ H NMR $\left(400 \mathrm{MHz}, \mathrm{CDCl}_{3}, \delta\right): 8.09-7.97(\mathrm{~m}, 2 \mathrm{H}), 7.93-7.82(\mathrm{~m}, 1 \mathrm{H}), 7.65(\mathrm{~d}, J=8.0 \mathrm{~Hz}$, $1 \mathrm{H}), 7.52(\mathrm{~s}, 1 \mathrm{H}), 7.42(\mathrm{~s}, 1 \mathrm{H}), 7.35-7.27(\mathrm{~m}, 1 \mathrm{H}), 4.11(\mathrm{q}, J=7.1 \mathrm{~Hz}, 2 \mathrm{H}), 3.36(\mathrm{dd}, J=14.2$, $1.4 \mathrm{~Hz}, 1 \mathrm{H}), 2.86(\mathrm{dd}, J=14.2,1.4 \mathrm{~Hz}, 1 \mathrm{H}), 2.47(\mathrm{~s}, 3 \mathrm{H}), 2.00-1.69(\mathrm{~m}, 2 \mathrm{H}), 1.30-1.18(\mathrm{~m}$, $2 \mathrm{H}), 1.14(\mathrm{t}, J=7.1 \mathrm{~Hz}, 3 \mathrm{H}), 1.09-0.99(\mathrm{~m}, 2 \mathrm{H}), 0.79(\mathrm{t}, J=7.1 \mathrm{~Hz}, 3 \mathrm{H})$.

${ }^{13}$ C NMR (101 MHz, $\left.\mathrm{CDCl}_{3}, \delta\right): 194.5,171.4,144.9,139.7,139.3,136.0,131.8,129.9$ (q, $J=$ $33.8 \mathrm{~Hz}), 129.8$ (q, $J=30.7 \mathrm{~Hz}), 130.4,128.5(2 \mathrm{C}), 128.3,124.8,123.44$ (q, $J=273.6 \mathrm{~Hz})$, 123.4 (hept, $J=4.7 \mathrm{~Hz}), 123.38$ (q, $J=272.0 \mathrm{~Hz}$ ), 123.2, 61.4, 58.7, 34.8, 33.3, 26.9, 22.9, $22.0,14.0,13.7$.

${ }^{19}$ F NMR $\left(376 \mathrm{MHz}, \mathrm{CDCl}_{3}, \delta\right):-61.0,-62.8$.

HRMS (ESI): Calculated for $\mathrm{C}_{27} \mathrm{H}_{27} \mathrm{~F}_{6} \mathrm{O}_{3}(\mathrm{M}+\mathrm{H})^{+}$513.1864, found 513.1858.

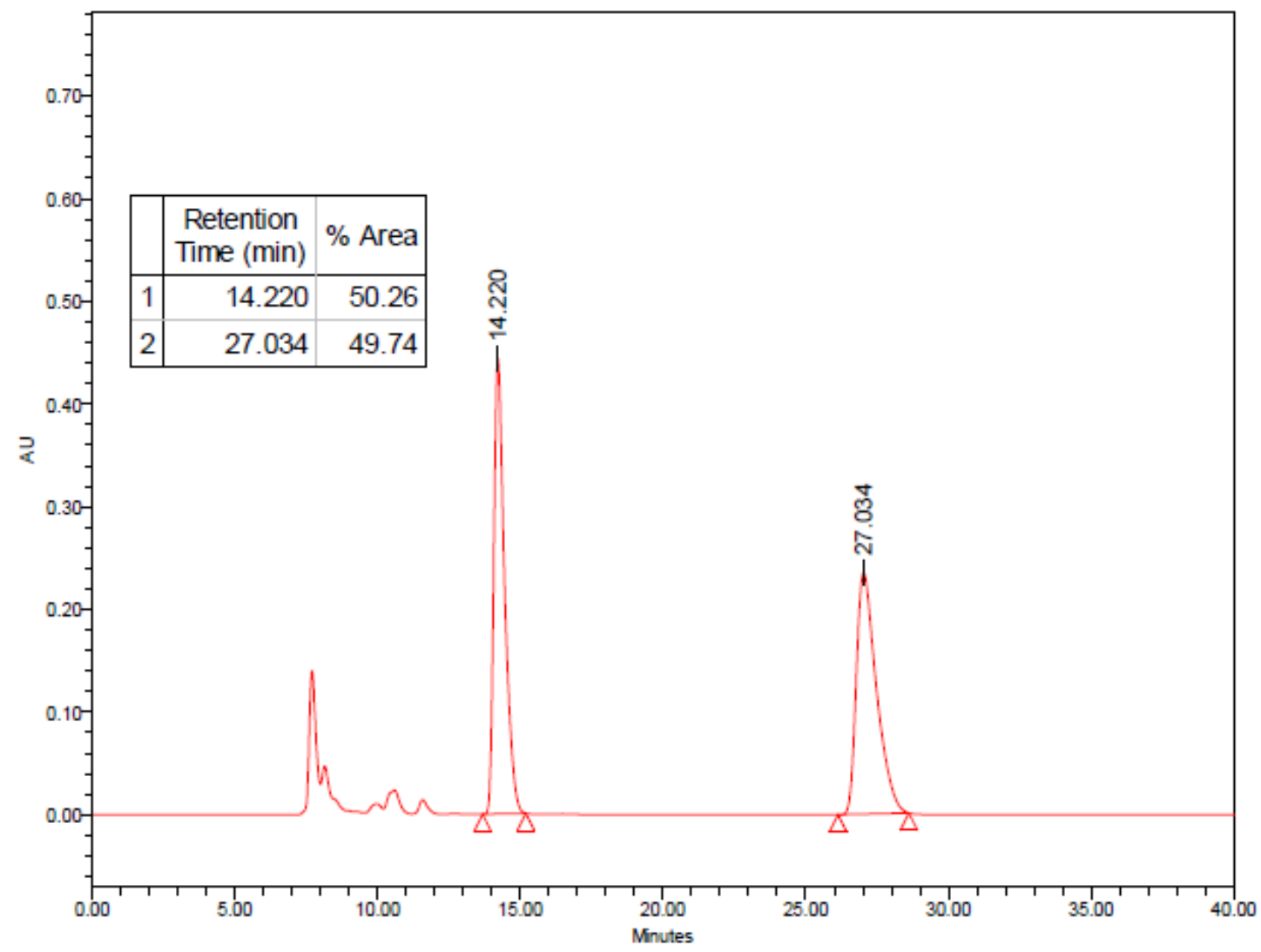




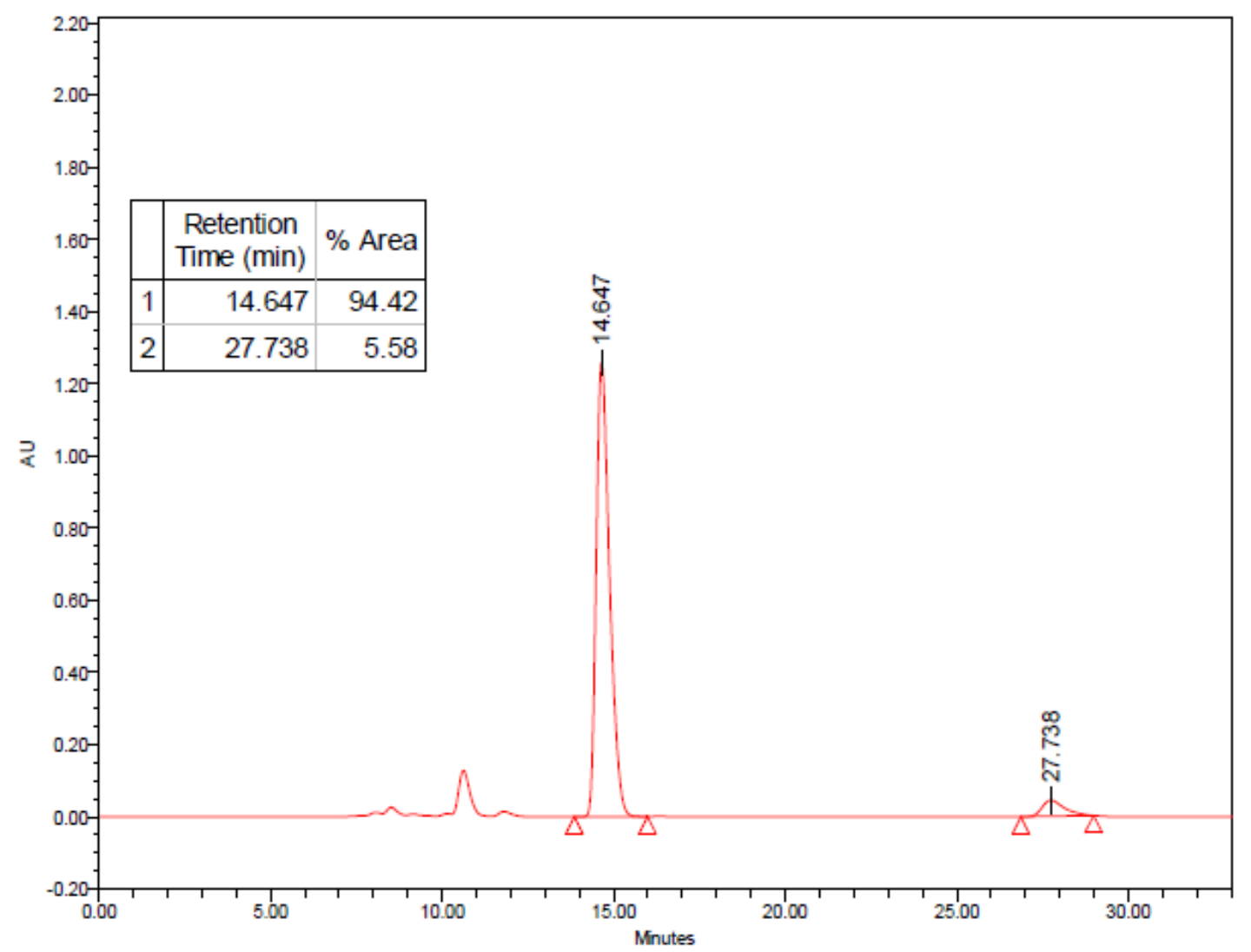

Ethyl (R,E)-4-(2,4-bis(trifluoromethyl)benzylidene)-2-butyl-7-methoxy-1-oxo-1,2,3,4tetrahydronaphthalene-2-carboxylate $(2 \mathrm{j})$<smiles>CCCC1(OCC)CC(=Cc2ccc(C(F)(F)F)cc2C(F)(F)F)c2ccc(OC)cc2C1=O</smiles>

Yellow crystals (69 mg, $87 \%$ ) obtained from the general procedure (3) using $\mathbf{S}_{\mathbf{6}}$ (70 $\mathrm{mg}, 0.15$ $\mathrm{mmol}$ ) and 4-methoxyphenylboronic acid (46 mg, $0.30 \mathrm{mmol})$.

$\mathbf{R}_{\mathbf{f}}=0.39$ (petroleum ether/EtOAc 9:1).

HPLC (Chiralpak ID hexane $/ \mathrm{PrOH}$ 95:5, $0.5 \mathrm{~mL} / \mathrm{min}$ ): $\mathrm{Rt}=19.8 \mathrm{~min}$ (major), $22.3 \mathrm{~min}$ (minor).

m.p. $=106^{\circ} \mathrm{C}($ dec. $)$

$[\boldsymbol{\alpha}]_{\mathbf{D}} \mathbf{2 0}^{\mathbf{2 0}}=+123\left(\mathrm{c}=1.5, \mathrm{CHCl}_{3}\right)$ for an enantiomeric excess of $88 \%$.

${ }^{1} \mathbf{H}$ NMR $\left(400 \mathrm{MHz}, \mathrm{CDCl}_{3}, \delta\right): 7.91(\mathrm{~s}, 1 \mathrm{H}), 7.79(\mathrm{~d}, J=7.9 \mathrm{~Hz}, 1 \mathrm{H}), 7.66-7.56(\mathrm{~m}, 2 \mathrm{H}), 7.48$ $(\mathrm{d}, J=2.8 \mathrm{~Hz}, 1 \mathrm{H}), 7.24(\mathrm{~s}, 1 \mathrm{H}), 7.12(\mathrm{dd}, J=8.8,2.8 \mathrm{~Hz}, 1 \mathrm{H}), 4.12-3.96(\mathrm{~m}, 2 \mathrm{H}), 3.83(\mathrm{~s}$, $3 \mathrm{H}), 3.29$ (dd, $J=14.3,1.4 \mathrm{~Hz}, 1 \mathrm{H}), 2.78$ (dd, $J=14.3,1.4 \mathrm{~Hz}, 1 \mathrm{H}), 1.99-1.63$ (m, 2H), $1.23-$ $1.11(\mathrm{~m}, 2 \mathrm{H}), 1.07(\mathrm{t}, J=7.1 \mathrm{~Hz}, 3 \mathrm{H}), 1.03-0.95(\mathrm{~m}, 2 \mathrm{H}), 0.73(\mathrm{t}, J=7.1 \mathrm{~Hz}, 3 \mathrm{H})$.

${ }^{13} \mathbf{C}$ NMR $\left(101 \mathrm{MHz}, \mathrm{CDCl}_{3}, \delta\right): 194.9,171.3,160.5,139.5,135.3,132.8,132.0,131.8,129.75$ $(\mathrm{q}, J=33.6 \mathrm{~Hz}), 129.74(\mathrm{q}, J=30.5 \mathrm{~Hz}), 128.5(\mathrm{q}, J=3.2 \mathrm{~Hz}), 126.2,123.46(\mathrm{q}, J=274.3$ $\mathrm{Hz}), 123.44$ (q, $J=272.3 \mathrm{~Hz}), 123.3$ (hept, $J=4.4 \mathrm{~Hz}), 122.4,121.7,109.7,61.4,58.6,55.6$, $34.9,33.4,26.9,22.9,13.9,13.7$.

${ }^{19}$ F NMR $\left(376 \mathrm{MHz}, \mathrm{CDCl}_{3}, \delta\right):-61.1,-62.8$.

HRMS (ESI): Calculated for $\mathrm{C}_{27} \mathrm{H}_{27} \mathrm{~F}_{6} \mathrm{O}_{4}(\mathrm{M}+\mathrm{H})^{+}$529.1814, found 529.1809. 

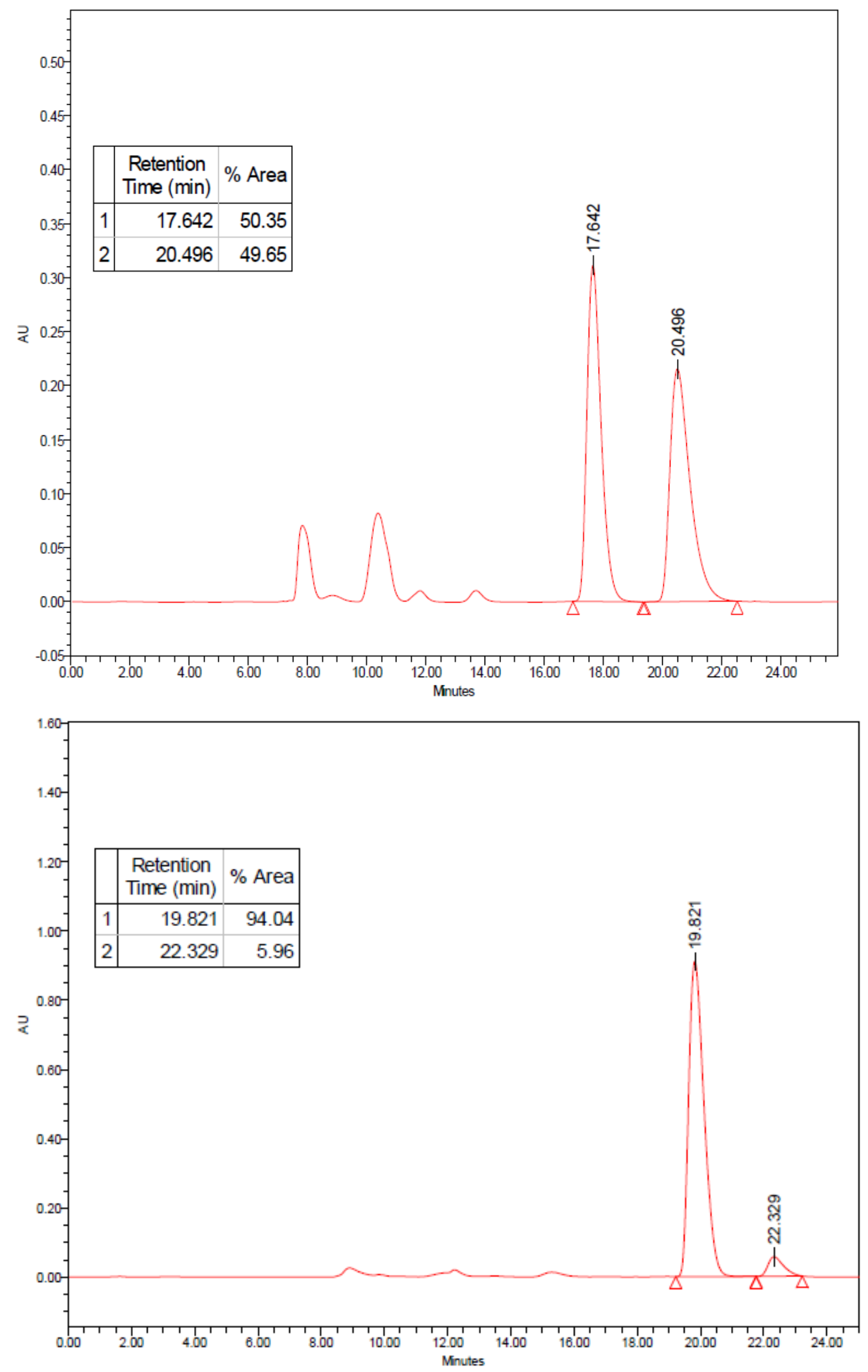
<smiles>CCOC[C@]1(NC(C)=O)C/C(=C\c2ccc(C(F)(F)F)cc2C(F)(F)F)c2ccccc2C1=O</smiles>

Orange oil (64 mg, $86 \%$ ) obtained from the general procedure (3) using $\mathbf{S}_{\mathbf{8}}$ (70 $\mathrm{mg}, 0.15 \mathrm{mmol}$ ) and phenylboronic acid (37 $\mathrm{mg}, 0.15 \mathrm{mmol})$.

$\mathbf{R}_{\mathbf{f}}=0.38$ (petroleum ether/EtOAc 6:4).

HPLC (Chiralpak ID hexane/iPrOH 90:10, $1.0 \mathrm{~mL} / \mathrm{min}$ ): Rt = $14.2 \mathrm{~min}$ (major), $22.4 \mathrm{~min}$ (minor).

$[\boldsymbol{\alpha}]_{\mathbf{D}}{ }^{\mathbf{2 0}}=+32\left(\mathrm{c}=1.5, \mathrm{CHCl}_{3}\right)$ for an enantiomeric excess of $92 \%$.

${ }^{1} \mathbf{H}$ NMR $\left(400 \mathrm{MHz}, \mathrm{CDCl}_{3}, \delta\right): 8.12(\mathrm{dd}, J=7.9,1.4 \mathrm{~Hz}, 1 \mathrm{H}), 7.96(\mathrm{~s}, 1 \mathrm{H}), 7.91-7.81(\mathrm{~m}, 2 \mathrm{H})$, $7.72(\mathrm{~d}, J=7.9 \mathrm{~Hz}, 1 \mathrm{H}), 7.70-7.64(\mathrm{~m}, 1 \mathrm{H}), 7.49(\mathrm{~m}, 2 \mathrm{H}), 6.94(\mathrm{~s}, 1 \mathrm{H}), 4.18(\mathrm{dq}, J=10.7,7.1$ $\mathrm{Hz}, 1 \mathrm{H}), 4.01(\mathrm{dq}, J=10.7,7.1 \mathrm{~Hz}, 1 \mathrm{H}), 3.71(\mathrm{~d}, J=15.0 \mathrm{~Hz}, 1 \mathrm{H}), 3.25(\mathrm{dd}, J=15.0,2.6 \mathrm{~Hz}$, $1 \mathrm{H}), 2.04(\mathrm{~s}, 3 \mathrm{H}), 1.06(\mathrm{t}, J=7.1 \mathrm{~Hz}, 3 \mathrm{H})$.

${ }^{13}$ C NMR (101 MHz, $\left.\mathrm{CDCl}_{3}, \delta\right): 189.6,169.7,167.8,140.0,139.3,134.7$ (2C), 131.9, 130.0, $129.9(\mathrm{q}, J=33.6 \mathrm{~Hz}), 129.7(\mathrm{q}, J=30.5 \mathrm{~Hz}), 129.2,128.7(2 \mathrm{C}), 124.4,123.5(\mathrm{q}, J=273.7$ $\mathrm{Hz}), 123.4$ (hept, $J=4.4 \mathrm{~Hz}), 123.3(\mathrm{q}, J=272.2 \mathrm{~Hz}), 122.4,66.3,62.8,35.2,23.1,13.6$.

${ }^{19}$ F NMR $\left(376 \mathrm{MHz}, \mathrm{CDCl}_{3}, \delta\right):-61.2,-62.8$.

HRMS (ESI): Calculated for $\mathrm{C}_{24} \mathrm{H}_{20} \mathrm{~F}_{6} \mathrm{NO}_{4}(\mathrm{M}+\mathrm{H})^{+}$500.1297, found 500.1290.

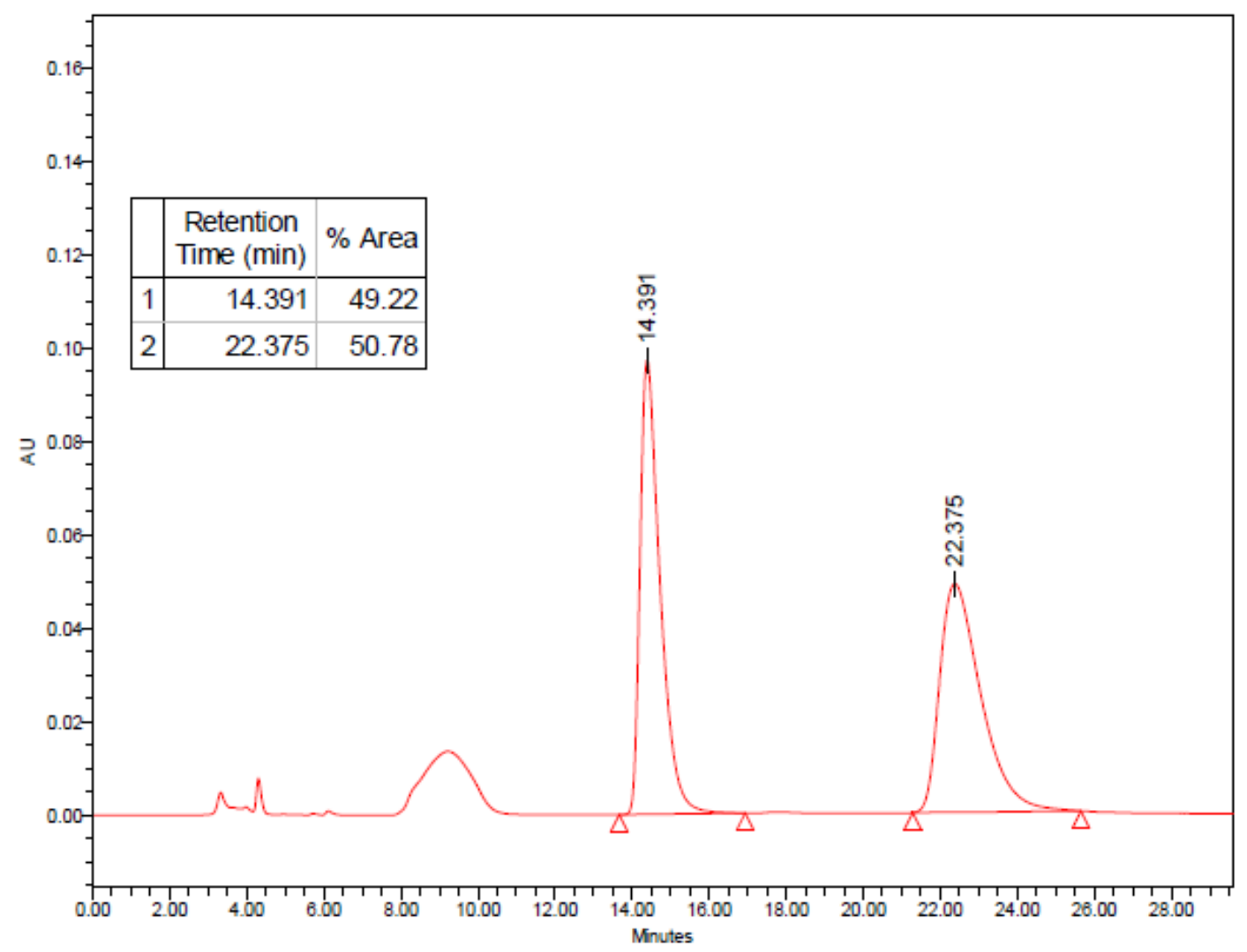




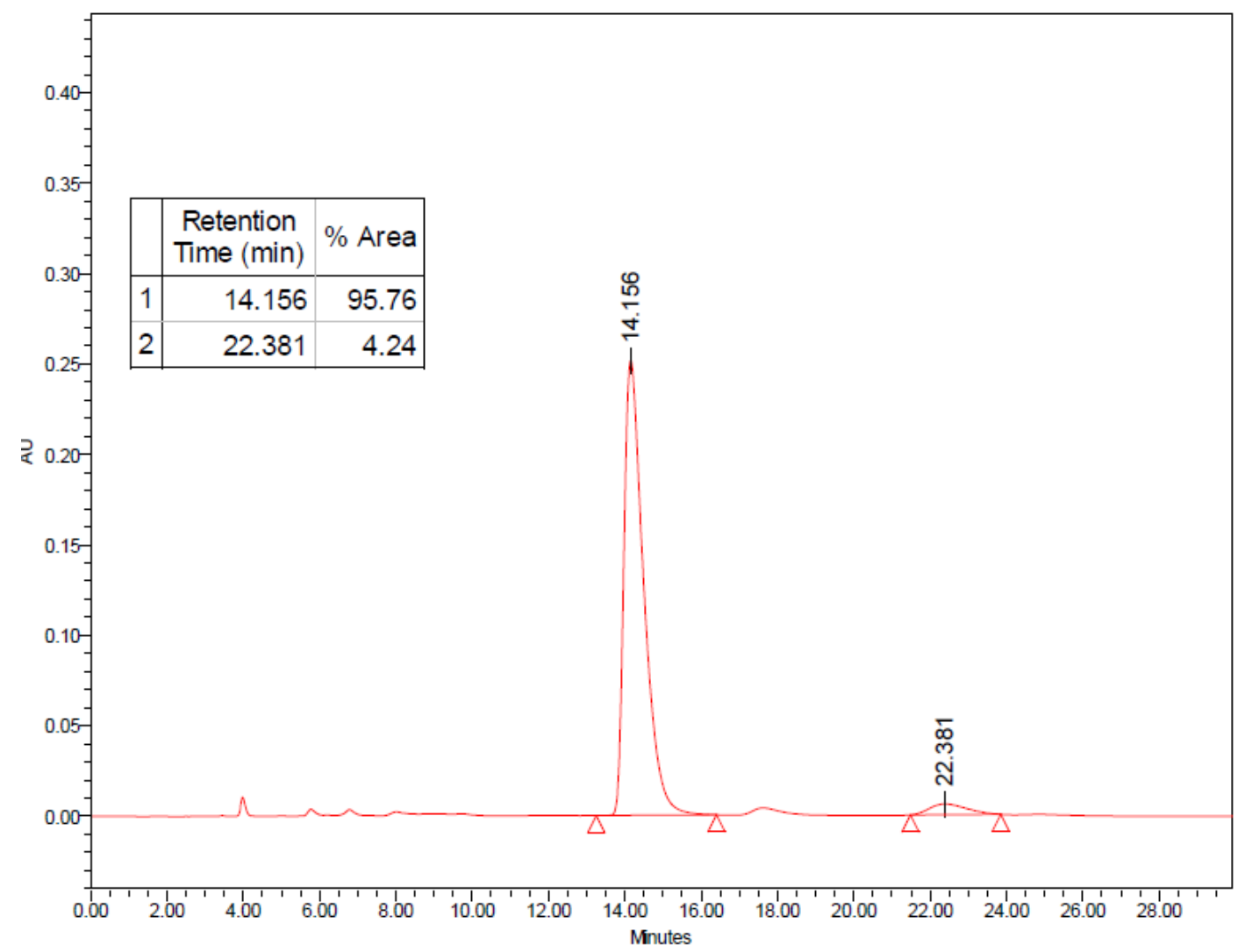

Ethyl (S,E)-2-acetamido-4-(2,4-bis(trifluoromethyl)benzylidene)-6-chloro-1-oxo-1,2,3,4tetrahydronaphthalene-2-carboxylate (2l)<smiles>CCOC(=O)N[C@@]1(C(=O)OCC)C/C(=C\c2ccc(C(F)(F)F)cc2C(F)(F)F)c2cc(Cl)ccc2C1=O</smiles>

Yellow oil (70 mg, $88 \%$ ) obtained from the general procedure (3) using $\mathbf{S}_{\mathbf{8}}$ (70 $\mathrm{mg}, 0.15 \mathrm{mmol}$ ) and 3-chlorophenylboronic acid (47 $\mathrm{mg}, 0.30 \mathrm{mmol})$.

$\mathbf{R}_{\mathbf{f}}=0.37$ (petroleum ether/EtOAc 6:4).

HPLC (Chiralpak ID hexane $/ \mathrm{iPrOH} 90: 10,1.0 \mathrm{~mL} / \mathrm{min}$ ): $\mathrm{Rt}=14.2 \mathrm{~min}$ (major), $21.5 \mathrm{~min}$ (minor).

$[\boldsymbol{\alpha}]_{\mathbf{D}}{ }^{\mathbf{2 0}}=+42\left(\mathrm{c}=1.3, \mathrm{CHCl}_{3}\right)$ for an enantiomeric excess of $89 \%$.

${ }^{1}$ H NMR $\left(400 \mathrm{MHz}, \mathrm{CDCl}_{3}, \delta\right): 8.07(\mathrm{~d}, J=8.4 \mathrm{~Hz}, 1 \mathrm{H}), 7.98(\mathrm{~s}, 1 \mathrm{H}), 7.87(\mathrm{~d}, J=8.1 \mathrm{~Hz}, 1 \mathrm{H})$, $7.79(\mathrm{~d}, J=2.0 \mathrm{~Hz}, 1 \mathrm{H}), 7.63(\mathrm{~d}, J=8.1 \mathrm{~Hz}, 1 \mathrm{H}), 7.45(\mathrm{dd}, J=8.4,1.9 \mathrm{~Hz}, 1 \mathrm{H}), 7.43(\mathrm{~s}, 1 \mathrm{H})$, $6.89(\mathrm{~s}, 1 \mathrm{H}), 4.20(\mathrm{dq}, J=10.7,7.1 \mathrm{~Hz}, 1 \mathrm{H}), 4.01(\mathrm{dq}, J=10.7,7.1 \mathrm{~Hz}, 1 \mathrm{H}), 3.56(\mathrm{~d}, J=15.0$ $\mathrm{Hz}, 1 \mathrm{H}), 3.33$ (dd, $J=15.0,2.6 \mathrm{~Hz}, 1 \mathrm{H}), 2.03$ (s, 3H), 1.07 (t, $J=7.1 \mathrm{~Hz}, 3 \mathrm{H})$.

${ }^{13}$ C NMR (101 MHz, $\left.\mathrm{CDCl}_{3}, \delta\right): 188.7,169.8,167.9,141.5,141.5,138.8,134.0,132.0,130.5$, $130.4(\mathrm{q}, J=33.2 \mathrm{~Hz}), 130.0(\mathrm{q}, J=30.5 \mathrm{~Hz}), 129.6,128.9(\mathrm{q}, J=3.5 \mathrm{~Hz}), 128.7,124.6,123.7$, 123.5 (hept, $J=4.9 \mathrm{~Hz}), 123.38$ (q, $J=274.2 \mathrm{~Hz}), 123.36$ (q, $J=272.2 \mathrm{~Hz}), 66.2,63.2,35.3$, 23.1, 13.7.

${ }^{19}$ F NMR $\left(376 \mathrm{MHz}, \mathrm{CDCl}_{3}, \delta\right):-61.1,-62.9$.

HRMS (ESI): Calculated for $\mathrm{C}_{24} \mathrm{H}_{19} \mathrm{~F}_{6} \mathrm{NO}_{4} \mathrm{Cl}(\mathrm{M}+\mathrm{H})^{+} 534.0907$, found 534.0901. 

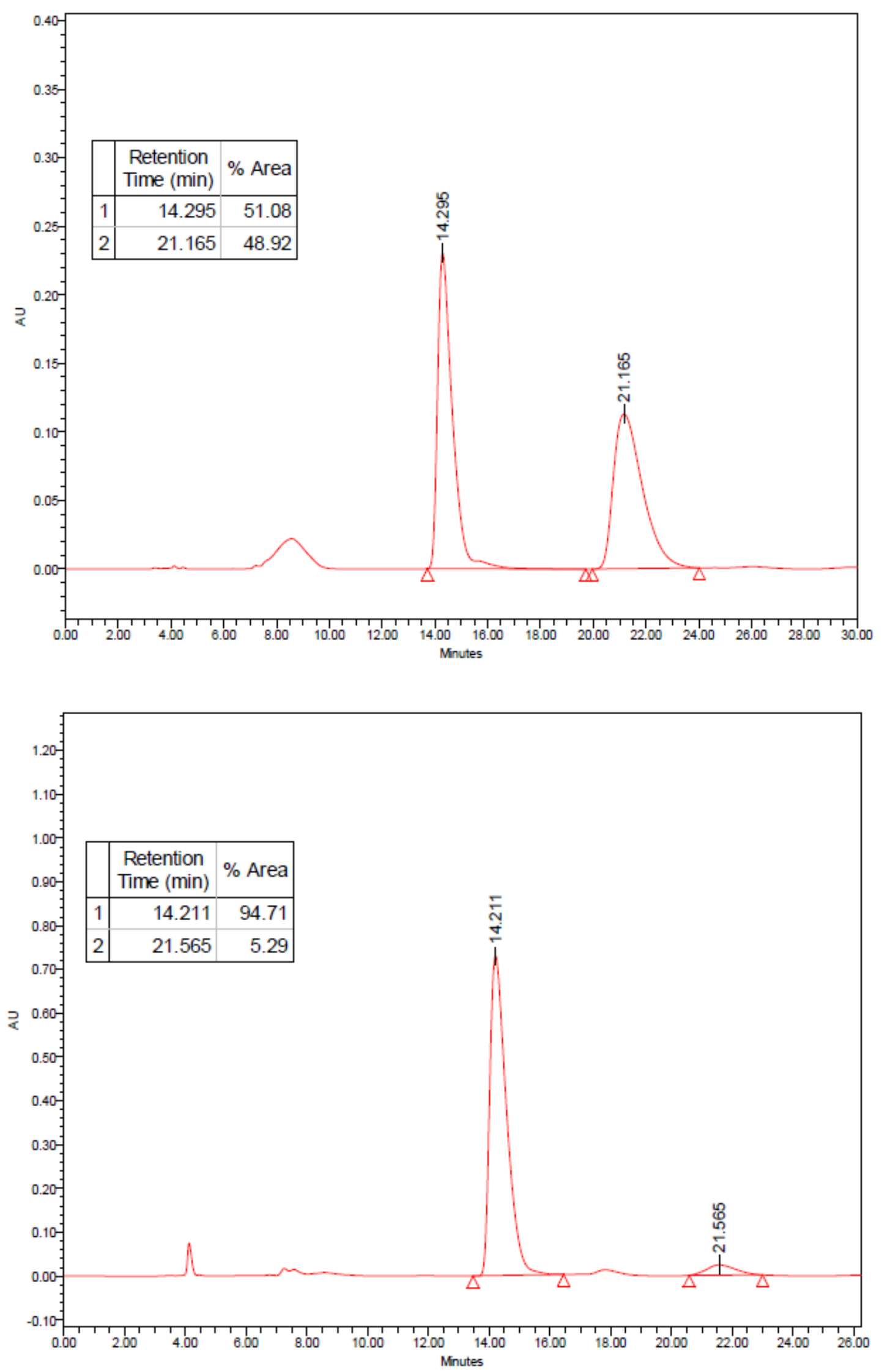
Ethyl (S,E)-2-acetamido-4-(2,4-bis(trifluoromethyl)benzylidene)-5-fluoro-1-oxo-1,2,3,4tetrahydronaphthalene-2-carboxylate $(2 \mathrm{~m})$<smiles>CCO[C@H]1C/C(=C\c2ccc(C(F)(F)F)cc2C(F)(F)F)c2c(F)cccc2C1=O</smiles>

Colorless oil (65 mg, $84 \%$ ) obtained from the general procedure (3) using $\mathbf{S}_{\mathbf{8}}$ (70 $\mathrm{mg}, 0.15$ $\mathrm{mmol}$ ) and 2-fluorophenylboronic acid (42 $\mathrm{mg}, 0.30 \mathrm{mmol})$.

$\mathbf{R}_{\mathbf{f}}=0.31$ (petroleum ether/EtOAc 6:4).

HPLC (Chiralpak ID hexane $/ \mathrm{iPrOH}$ 90:10, $1.0 \mathrm{~mL} / \mathrm{min}$ ): $\mathrm{Rt}=13.2 \mathrm{~min}$ (major), $19.3 \mathrm{~min}$ (minor).

$[\boldsymbol{\alpha}]_{\mathbf{D}} \mathbf{2 0}^{\mathbf{2 0}}=+35\left(\mathrm{c}=1.1, \mathrm{CHCl}_{3}\right)$ for an enantiomeric excess of $94 \%$.

${ }^{1} \mathbf{H}$ NMR $\left(400 \mathrm{MHz}, \mathrm{CDCl}_{3}, \delta\right): 8.02-7.94(\mathrm{~m}, 2 \mathrm{H}), 7.85(\mathrm{~d}, J=8.2 \mathrm{~Hz}, 1 \mathrm{H}), 7.76(\mathrm{~s}, 1 \mathrm{H}), 7.59$ $(\mathrm{d}, J=8.2 \mathrm{~Hz}, 1 \mathrm{H}), 7.43$ (s, 1H), 7.46-7.39 (m, 1H), $6.82(\mathrm{~s}, 1 \mathrm{H}), 4.22$ (dq, $J=10.7,7.1 \mathrm{~Hz}$, $1 \mathrm{H}), 4.03(\mathrm{dq}, J=10.7,7.1 \mathrm{~Hz}, 1 \mathrm{H}), 3.44(\mathrm{~d}, J=14.8 \mathrm{~Hz}, 1 \mathrm{H}), 3.37(\mathrm{dd}, J=14.8,2.3 \mathrm{~Hz}, 1 \mathrm{H})$, $2.02(\mathrm{~s}, 3 \mathrm{H}), 1.09(\mathrm{t}, J=7.1 \mathrm{~Hz}, 3 \mathrm{H})$.

${ }^{13}$ C NMR (101 MHz, $\left.\mathrm{CDCl}_{3}, \delta\right): 188.9,169.6,168.2,159.9$ (d, $\left.J=253.6 \mathrm{~Hz}\right), 139.4,132.7$, $131.8,130.1(\mathrm{q}, J=33.2 \mathrm{~Hz}), 129.7(\mathrm{q}, J=30.4 \mathrm{~Hz}), 129.6(\mathrm{~d}, J=5.1 \mathrm{~Hz}), 129.6,128.7(\mathrm{q}, J$ $=3.7 \mathrm{~Hz}), 127.7(\mathrm{~d}, J=9.7 \mathrm{~Hz}), 124.9(\mathrm{~d}, J=3.4 \mathrm{~Hz}), 124.5,123.5$ (hept, $J=4.5 \mathrm{~Hz}), 123.4$ (q, $J=273.9 \mathrm{~Hz}, 2 \mathrm{C}), 122.4(\mathrm{~d}, J=24.6 \mathrm{~Hz}), 66.0,63.2,35.9,23.1,13.7$.

${ }^{19} \mathbf{F}$ NMR $\left(376 \mathrm{MHz}, \mathrm{CDCl}_{3}, \delta\right):-61.2,-62.8,-112.2$.

HRMS (ESI): Calculated for $\mathrm{C}_{24} \mathrm{H}_{19} \mathrm{~F}_{7} \mathrm{NO}_{4}(\mathrm{M}+\mathrm{H})^{+}$518.1202, found 518.1198.

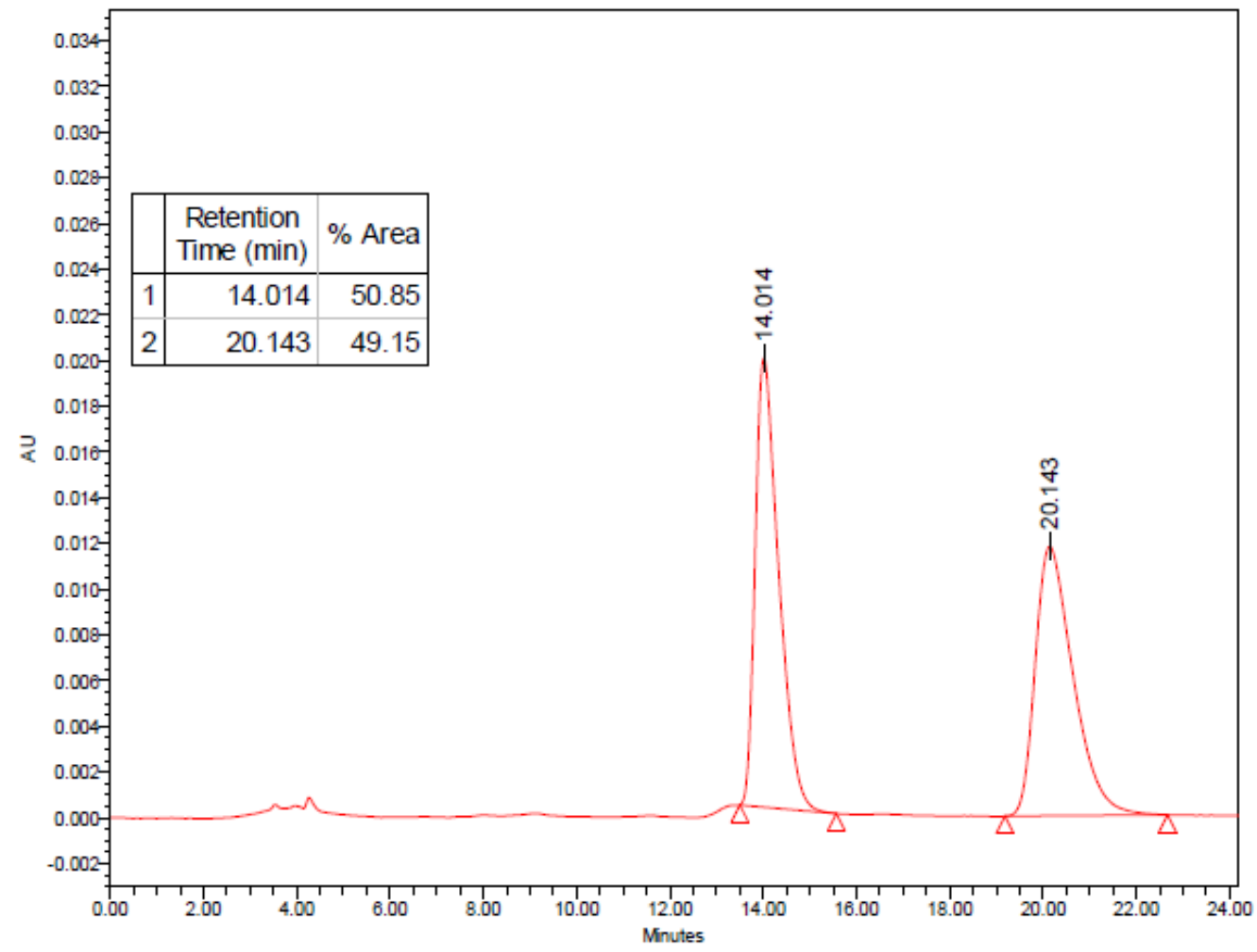




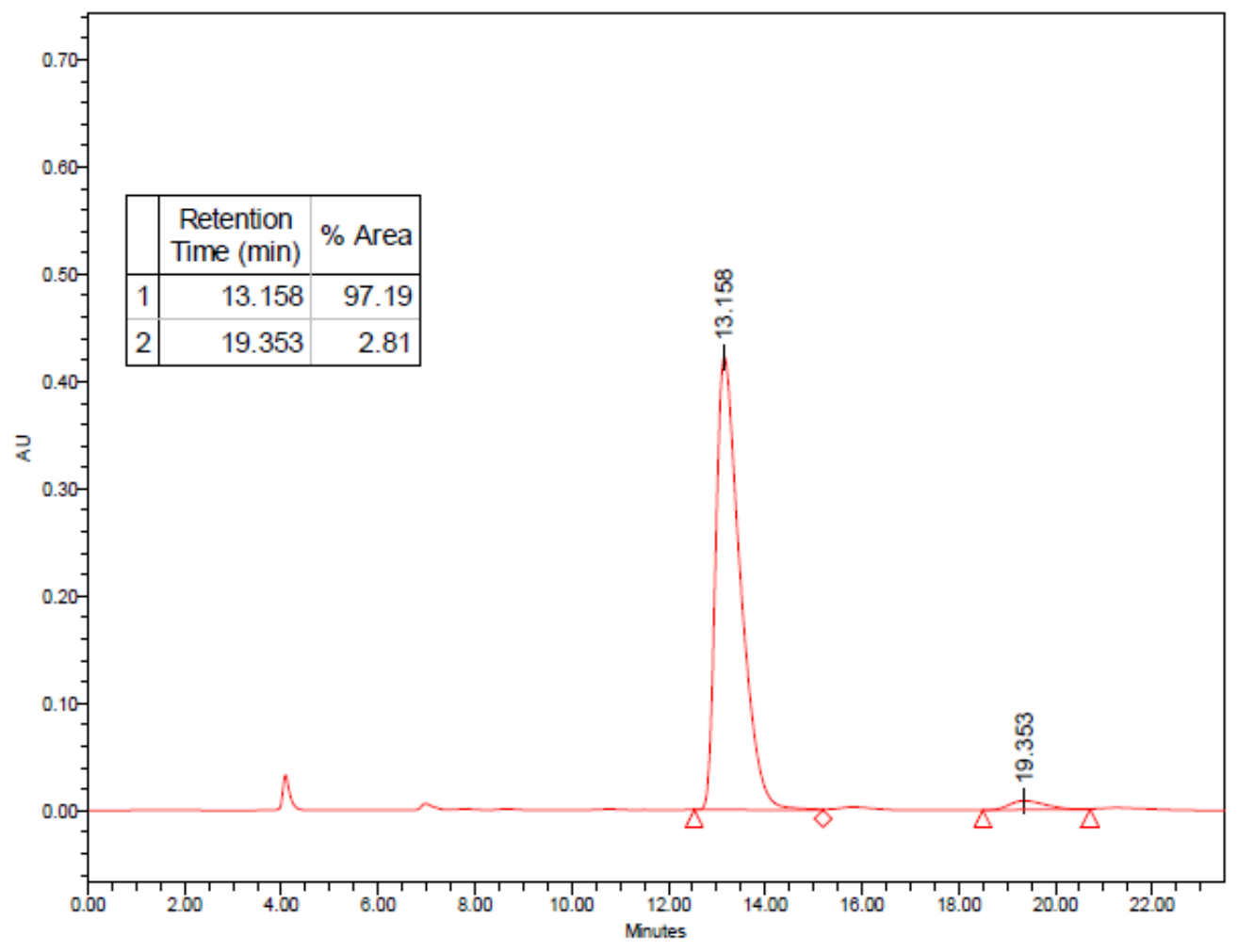

Ethyl (S,E)-2-acetamido-4-(2,4-bis(trifluoromethyl)benzylidene)-7-(tert-butyl)-1-oxo1,2,3,4-tetrahydronaphthalene-2-carboxylate (2n)<smiles>CCOC(=O)[C@]1(N)C/C(=C\c2ccc(C(F)(F)F)cc2C(F)(F)F)c2ccc(C(C)(C)C)cc2C1=O</smiles>

Colorless oil (72 mg, 87 \%) obtained from the general procedure (3) using $\mathbf{S}_{\mathbf{8}}$ (70 mg, 0.15 $\mathrm{mmol}$ ) and 4-tert-butylphenylboronic acid (54 mg, $0.30 \mathrm{mmol})$.

$\mathbf{R}_{\mathbf{f}}=0.43$ (petroleum ether/EtOAc 6:4).

HPLC (Chiralpak IE hexane $/ \mathrm{PrOH}$ 90:10, $1.0 \mathrm{~mL} / \mathrm{min}$ ): $\mathrm{Rt}=15.0 \mathrm{~min}$ (major), $17.8 \mathrm{~min}$ (minor).

$[\boldsymbol{\alpha}]_{\mathbf{D}}{ }^{\mathbf{2 0}}=+39\left(\mathrm{c}=1.3, \mathrm{CHCl}_{3}\right)$ for an enantiomeric excess of $96 \%$.

${ }^{1} \mathbf{H}$ NMR $\left(400 \mathrm{MHz}, \mathrm{CDCl}_{3}, \delta\right): 8.10(\mathrm{~d}, J=2.2 \mathrm{~Hz}, 1 \mathrm{H}), 7.95(\mathrm{~s}, 1 \mathrm{H}), 7.86(\mathrm{~d}, J=8.0 \mathrm{~Hz}, 1 \mathrm{H})$, $7.79(\mathrm{~d}, J=8.5 \mathrm{~Hz}, 1 \mathrm{H}), 7.72(\mathrm{~m}, 2 \mathrm{H}), 7.44(\mathrm{~s}, 1 \mathrm{H}), 6.98(\mathrm{~s}, 1 \mathrm{H}), 4.18(\mathrm{dq}, J=10.7,7.1 \mathrm{~Hz}$, $1 \mathrm{H}), 4.02(\mathrm{dq}, J=10.7,7.1 \mathrm{~Hz}, 1 \mathrm{H}), 3.72(\mathrm{~d}, J=15.0 \mathrm{~Hz}, 1 \mathrm{H}), 3.22(\mathrm{dd}, J=15.0,2.6 \mathrm{~Hz}, 1 \mathrm{H})$, $2.04(\mathrm{~s}, 3 \mathrm{H}), 1.36(\mathrm{~s}, 9 \mathrm{H}), 1.07(\mathrm{t}, J=7.1 \mathrm{~Hz}, 3 \mathrm{H})$.

${ }^{13}$ C NMR (101 MHz, $\left.\mathrm{CDCl}_{3}, \delta\right): 189.9,169.8,167.9,152.7,139.5,137.5,134.6,132.4,132.0$, $129.8(\mathrm{q}, J=34.0 \mathrm{~Hz}), 129.6,129.6(\mathrm{q}, J=30.5 \mathrm{~Hz}), 128.7(\mathrm{q}, J=3.5 \mathrm{~Hz}), 125.0,124.4,123.5$ $(\mathrm{q}, J=274.0 \mathrm{~Hz}), 123.4(\mathrm{q}, J=272.4 \mathrm{~Hz}), 123.3$ (hept, $J=4.6 \mathrm{~Hz}), 121.6,66.4,62.8,35.2$, $34.9,31.0(3 \mathrm{C}), 23.1,13.7$.

${ }^{19}$ F NMR (376 MHz, $\left.\mathrm{CDCl}_{3}, \delta\right):-61.2,-62.8$.

HRMS (ESI): Calculated for $\mathrm{C}_{28} \mathrm{H}_{28} \mathrm{~F}_{6} \mathrm{NO}_{4}(\mathrm{M}+\mathrm{H})^{+}$556.1923, found 556.1914. 

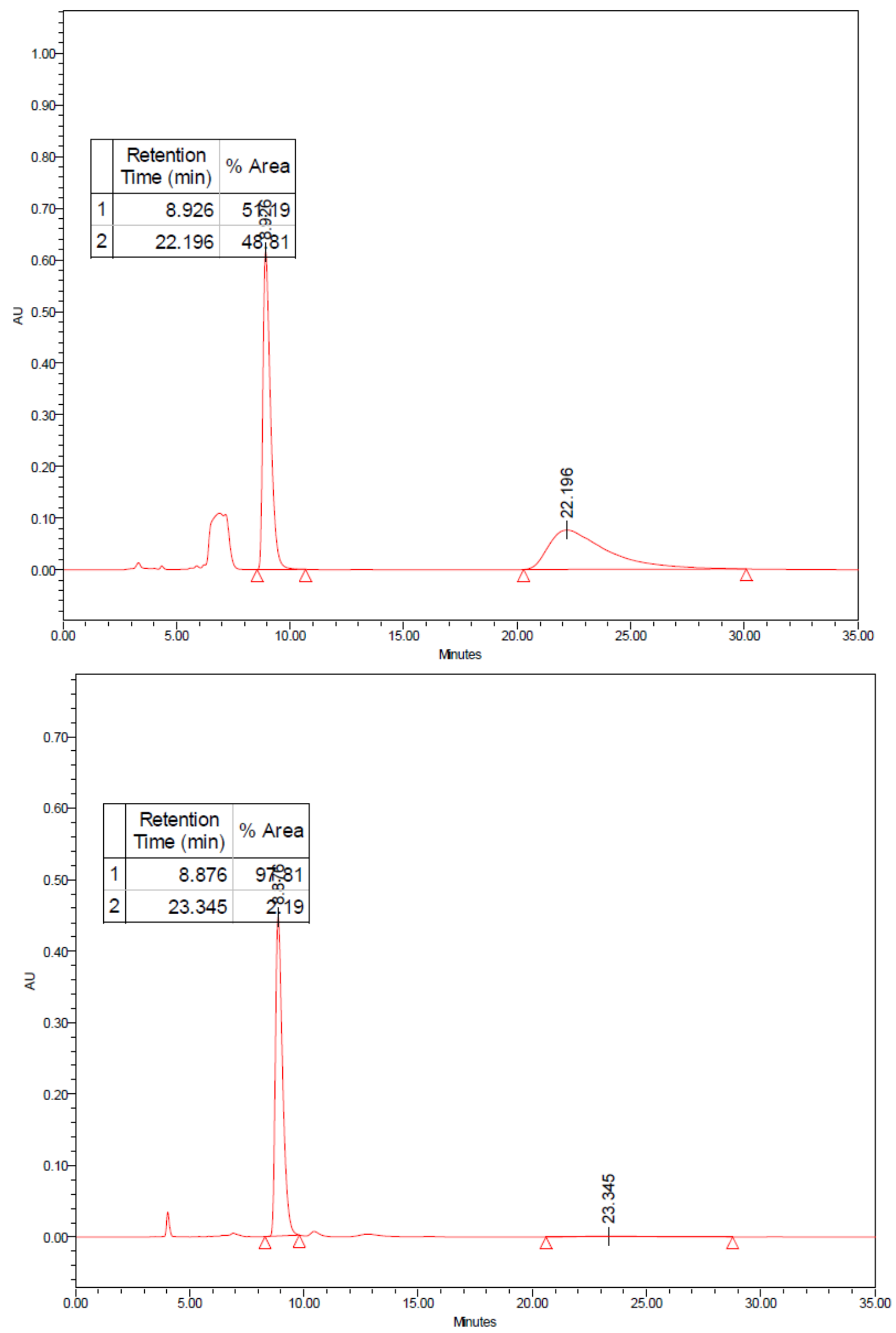
Ethyl (S,E)-2-acetamido-4-(2,4-bis(trifluoromethyl)benzylidene)-7-methyl-1-oxo-1,2,3,4tetrahydronaphthalene-2-carboxylate (2o)<smiles>CCOC(=O)[C@]1(NC(=O)CC)CC(=Cc2ccc(C(F)(F)F)cc2C(F)(F)F)c2ccc(C)cc2C1=O</smiles>

Orange oil (68 mg, $89 \%$ ) obtained from the general procedure (3) using $\mathbf{S}_{\mathbf{8}}$ (70 $\mathrm{mg}, 0.15 \mathrm{mmol}$ ) and 4-methylphenylboronic acid (41 $\mathrm{mg}, 0.30 \mathrm{mmol})$.

$\mathbf{R}_{\mathbf{f}}=0.38$ (petroleum ether/EtOAc 6:4).

HPLC (Chiralpak ID hexane/iPrOH 90:10, $1.0 \mathrm{~mL} / \mathrm{min}$ ): Rt = $14.5 \mathrm{~min}$ (major), $25.7 \mathrm{~min}$ (minor).

$[\boldsymbol{\alpha}]_{\mathbf{D}^{\mathbf{2 0}}}=+31\left(\mathrm{c}=1.85, \mathrm{CHCl}_{3}\right)$ for an enantiomeric excess of $94 \%$.

${ }^{1} \mathbf{H}$ NMR $\left(400 \mathrm{MHz}, \mathrm{CDCl}_{3}, \delta\right): 7.96(\mathrm{~d}, J=1.7 \mathrm{~Hz}, 1 \mathrm{H}), 7.91(\mathrm{~s}, 1 \mathrm{H}), 7.86(\mathrm{~d}, J=8.0 \mathrm{~Hz}, 1 \mathrm{H})$, $7.74(\mathrm{~d}, J=8.0 \mathrm{~Hz}, 1 \mathrm{H}), 7.71(\mathrm{~d}, J=8.0 \mathrm{~Hz}, 1 \mathrm{H}), 7.53-7.45(\mathrm{~m}, 1 \mathrm{H}), 7.42(\mathrm{~s}, 1 \mathrm{H}), 6.95(\mathrm{~s}$, $1 \mathrm{H}), 4.18(\mathrm{dq}, J=10.8,7.1 \mathrm{~Hz}, 1 \mathrm{H}), 4.00(\mathrm{dq}, J=10.8,7.1 \mathrm{~Hz}, 1 \mathrm{H}), 3.70(\mathrm{~d}, J=15.1 \mathrm{~Hz}, 1 \mathrm{H})$, $3.21(\mathrm{dd}, J=15.1,2.6 \mathrm{~Hz}, 1 \mathrm{H}), 2.44(\mathrm{~s}, 3 \mathrm{H}), 2.04(\mathrm{~s}, 3 \mathrm{H}), 1.07(\mathrm{t}, J=7.1 \mathrm{~Hz}, 3 \mathrm{H})$.

${ }^{13}$ C NMR (101 MHz, $\left.\mathrm{CDCl}_{3}, \delta\right): 189.9,169.8,167.9,139.5$ (2C), 137.5, 135.7, 134.6, 132.0, $129.8,129.8(\mathrm{q}, J=33.6 \mathrm{~Hz}), 129.6(\mathrm{q}, J=30.8 \mathrm{~Hz}), 128.7(2 \mathrm{C}), 124.4,123.5(\mathrm{q}, J=273.7$ $\mathrm{Hz}), 123.4$ (q, $J=272.3 \mathrm{~Hz}), 123.3$ (hept, $J=4.6 \mathrm{~Hz}), 121.5,66.3,62.8,35.2,23.0,21.1$, 13.6. ${ }^{19}$ F NMR $\left(376 \mathrm{MHz}, \mathrm{CDCl}_{3}, \delta\right):-61.3,-62.8$.

HRMS (ESI): Calculated for $\mathrm{C}_{25} \mathrm{H}_{22} \mathrm{~F}_{6} \mathrm{NO}_{4}(\mathrm{M}+\mathrm{H})^{+}$514.1453, found 514.1448.

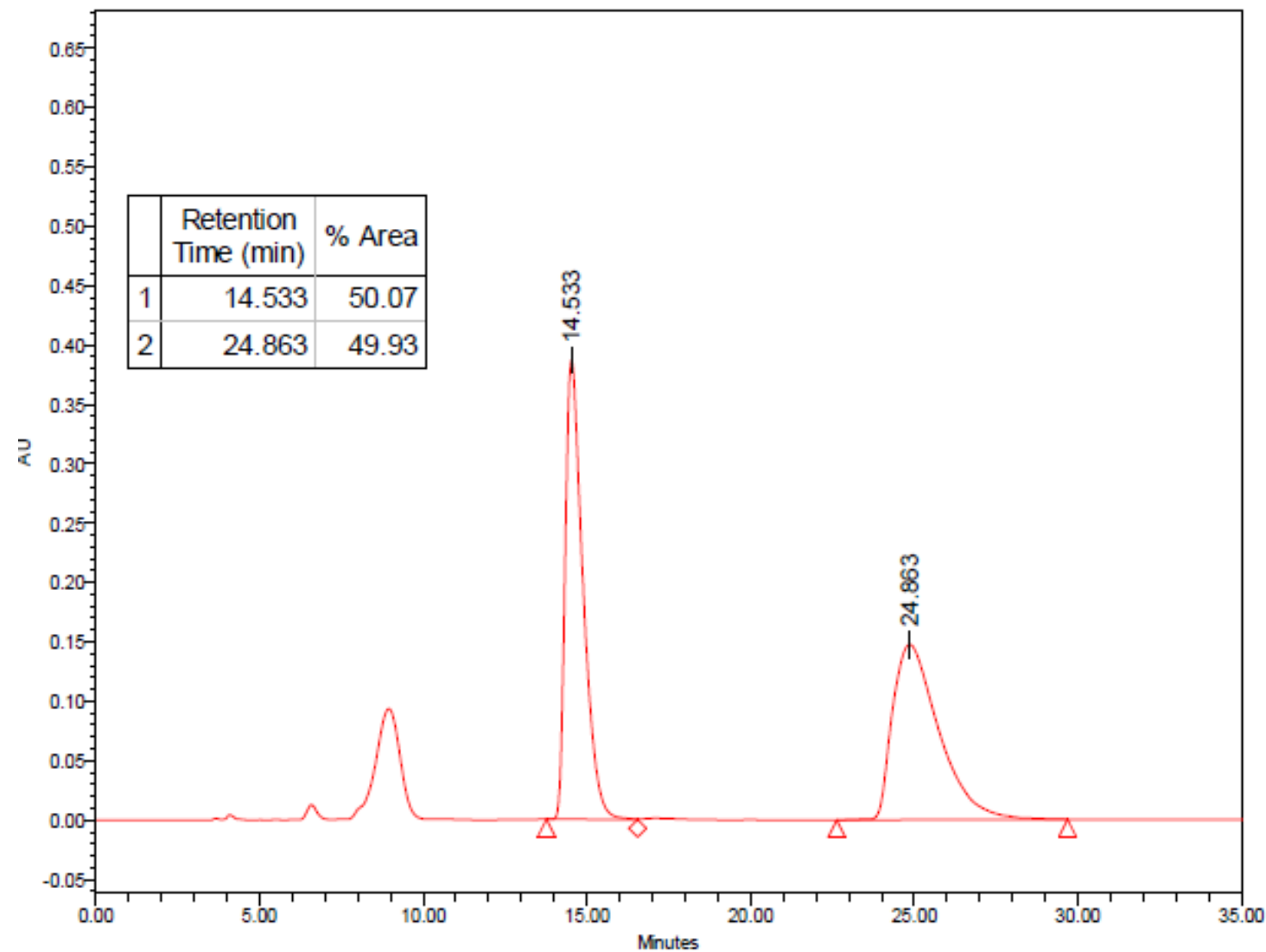




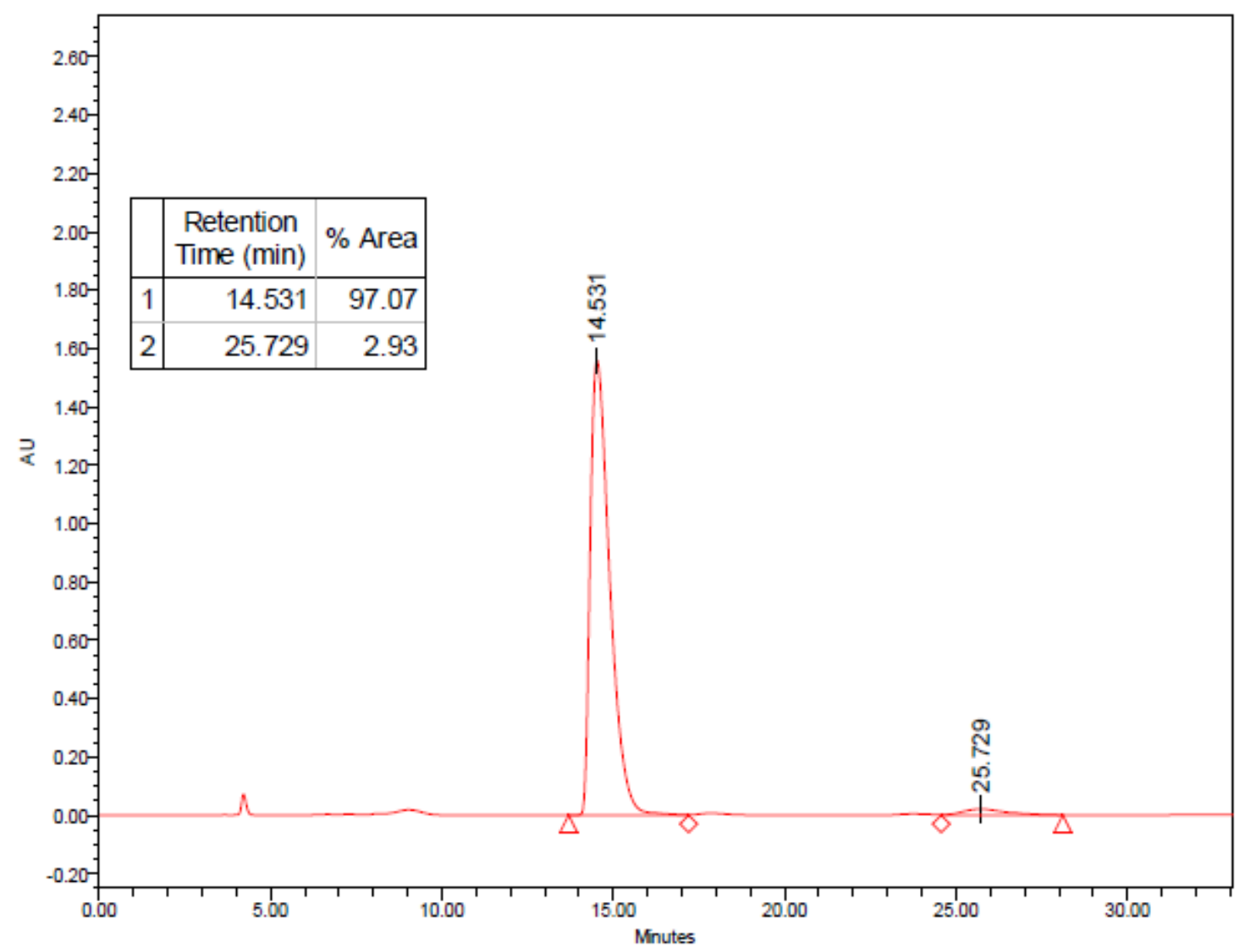

Ethyl (S,E)-2-acetamido-4-(2,4-bis(trifluoromethyl)benzylidene)-6-methoxy-1-oxo1,2,3,4-tetrahydronaphthalene-2-carboxylate $(2 p)$<smiles>CCOC(=O)N[C@@]1(C(=O)OCC)CC(=Cc2ccc(C(F)(F)F)cc2C(F)(F)F)c2cc(OC)ccc2C1=O</smiles>

Yellow oil (74 mg, $93 \%$ ) obtained from the general procedure (3) using $\mathbf{S}_{\mathbf{8}}$ (70 $\mathrm{mg}, 0.15 \mathrm{mmol}$ ) and 3-methoxyphenylboronic acid (46 $\mathrm{mg}, 0.30 \mathrm{mmol}$ ).

$\mathbf{R}_{\mathbf{f}}=0.34$ (petroleum ether/EtOAc 6:4).

HPLC (Chiralpak ID hexane $/ \mathrm{iPrOH} 85: 15,1.0 \mathrm{~mL} / \mathrm{min}$ ): $\mathrm{Rt}=18.3 \mathrm{~min}$ (major), $36.2 \mathrm{~min}$ (minor).

$[\boldsymbol{\alpha}]_{\mathbf{D}^{\mathbf{2 0}}}=+46\left(\mathrm{c}=1.6, \mathrm{CHCl}_{3}\right)$ for an enantiomeric excess of $91 \%$.

${ }^{1} \mathbf{H}$ NMR $\left(400 \mathrm{MHz}, \mathrm{CDCl}_{3}, \delta\right): 8.09(\mathrm{~d}, J=8.8 \mathrm{~Hz}, 1 \mathrm{H}), 7.96(\mathrm{~s}, 1 \mathrm{H}), 7.87(\mathrm{dd}, J=8.2,1.8$ $\mathrm{Hz}, 1 \mathrm{H}), 7.77(\mathrm{~d}, J=8.2 \mathrm{~Hz}, 1 \mathrm{H}), 7.44(\mathrm{~s}, 1 \mathrm{H}), 7.25(\mathrm{~d}, J=2.4 \mathrm{~Hz}, 1 \mathrm{H}), 7.03-6.96(\mathrm{~m}, 2 \mathrm{H})$, $4.18(\mathrm{dq}, J=10.7,7.1 \mathrm{~Hz}, 1 \mathrm{H}), 4.01(\mathrm{dq}, J=10.7,7.1 \mathrm{~Hz}, 1 \mathrm{H}), 3.94(\mathrm{~s}, 3 \mathrm{H}), 3.77(\mathrm{~d}, J=15.0$ $\mathrm{Hz}, 1 \mathrm{H}), 3.15(\mathrm{dd}, J=15.0,2.6 \mathrm{~Hz}, 1 \mathrm{H}), 2.02(\mathrm{~s}, 3 \mathrm{H}), 1.08(\mathrm{t}, J=7.1 \mathrm{~Hz}, 3 \mathrm{H})$.

${ }^{13}$ C NMR (101 MHz, $\left.\mathrm{CDCl}_{3}, \delta\right): 188.3,169.9,167.9,164.7,142.3,139.3,134.8,132.0,131.4$, 129.9 (q, $J=33.7 \mathrm{~Hz}), 129.6(\mathrm{q}, J=30.4 \mathrm{~Hz}), 128.8(\mathrm{q}, J=3.5 \mathrm{~Hz}), 123.7(\mathrm{q}, J=274.1 \mathrm{~Hz})$, 123.6, 123.4 (q, $J=272.3 \mathrm{~Hz}), 123.3$ (hept, $J=4.4 \mathrm{~Hz}$ ), 122.3, 115.6, 108.5, 66.2, 62.7, 55.7, $35.2,23.1,13.7$.

${ }^{19}$ F NMR $\left(376 \mathrm{MHz}, \mathrm{CDCl}_{3}, \delta\right):-61.2,-62.8$. 
HRMS (ESI): Calculated for $\mathrm{C}_{25} \mathrm{H}_{22} \mathrm{~F}_{6} \mathrm{NO}_{5}(\mathrm{M}+\mathrm{H})^{+}$530.1402, found 530.1388.
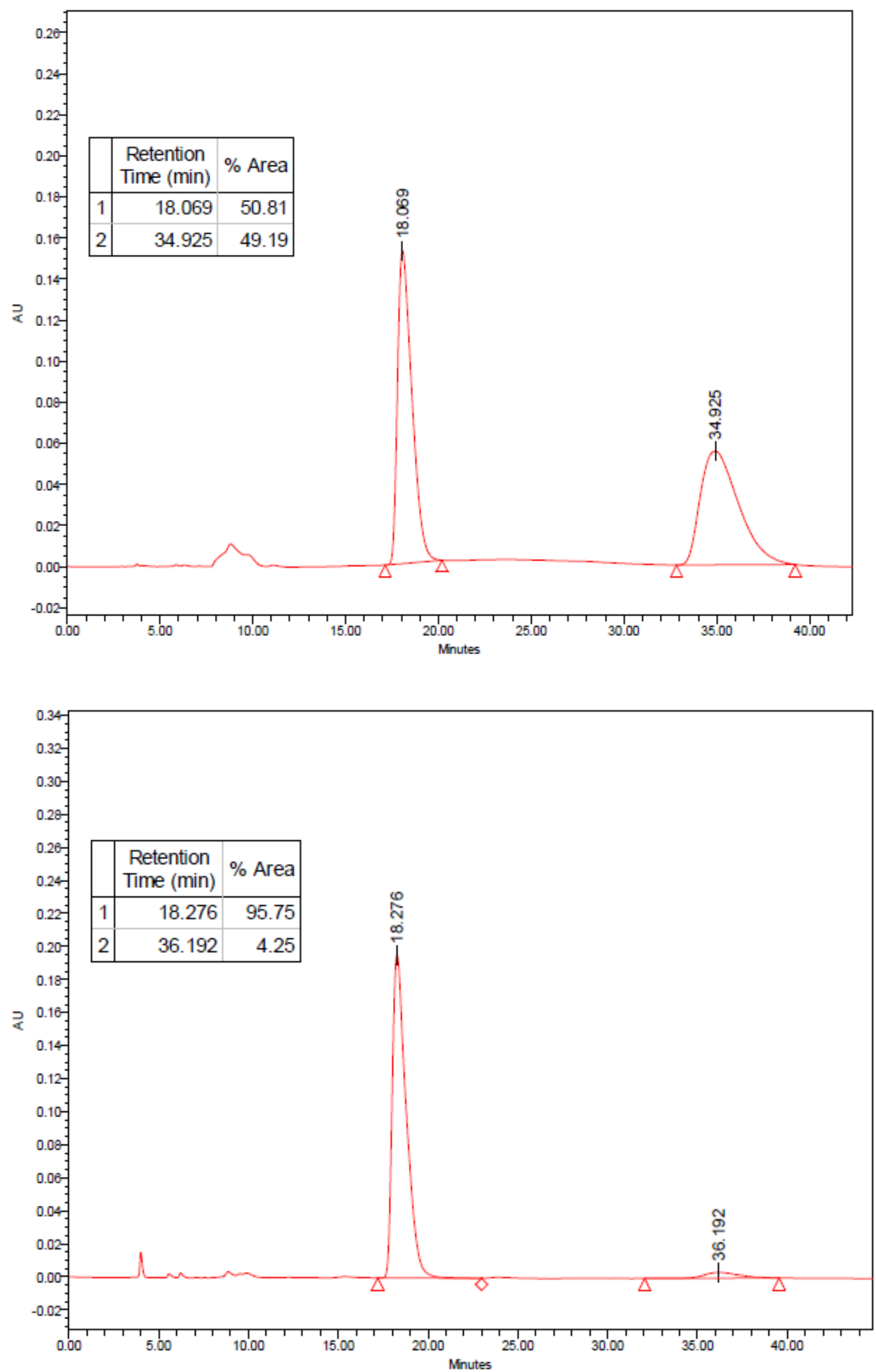
Ethyl (S,E)-4-(2,4-bis(trifluoromethyl)benzylidene)-2-((tert-butoxycarbonyl)amino)-1oxo-1,2,3,4-tetrahydronaphthalene-2-carboxylate (2q)<smiles>CCOC(=O)N[C@]1(C(=O)OCC)CC(=Cc2ccc(C(F)(F)F)cc2C(F)(F)F)c2ccccc2C1=O</smiles>

Brown oil (80 mg, $96 \%$ ) obtained from the general procedure (3) using $\mathbf{S}_{7}$ (79 $\mathrm{mg}, 0.15 \mathrm{mmol}$ ) and phenylboronic acid (37 $\mathrm{mg}, 0.30 \mathrm{mmol})$.

$\mathbf{R}_{\mathbf{f}}=0.26$ (petroleum ether/EtOAc 9:1).

HPLC (Chiralpak IE hexane $/ \mathrm{iPrOH}$ 93:7, $1.0 \mathrm{~mL} / \mathrm{min}$ ): $\mathrm{Rt}=12.8 \mathrm{~min}$ (major), $15.4 \mathrm{~min}$ (minor).

$[\boldsymbol{\alpha}]_{\mathbf{D}^{20}}=+29\left(\mathrm{c}=1.9, \mathrm{CHCl}_{3}\right)$ for an enantiomeric excess of $87 \%$.

${ }^{1}$ H NMR $\left(400 \mathrm{MHz}, \mathrm{C}_{6} \mathrm{D}_{6}, 70{ }^{\circ} \mathrm{C}, \delta\right): 8.08(\mathrm{dd}, J=7.9,1.6 \mathrm{~Hz}, 1 \mathrm{H}), 7.85(\mathrm{~s}, 1 \mathrm{H}), 7.61(\mathrm{~d}, J=$ $7.9 \mathrm{~Hz}, 1 \mathrm{H}), 7.54(\mathrm{~d}, J=7.9 \mathrm{~Hz}, 1 \mathrm{H}), 7.40-7.33(\mathrm{~m}, 2 \mathrm{H}), 7.10(\mathrm{td}, J=7.9,1.6 \mathrm{~Hz}, 1 \mathrm{H}), 6.97$ $(\mathrm{t}, J=7.9 \mathrm{~Hz}, 1 \mathrm{H}), 6.22(\mathrm{~s}, 1 \mathrm{H}), 3.96(\mathrm{~d}, J=15.3 \mathrm{~Hz}, 1 \mathrm{H}), 3.93-3.83(\mathrm{~m}, 1 \mathrm{H}), 3.73(\mathrm{dq}, J=$ $10.6,7.1 \mathrm{~Hz}, 1 \mathrm{H}), 2.99(\mathrm{~d}, J=15.3 \mathrm{~Hz}, 1 \mathrm{H}), 1.30(\mathrm{~s}, 9 \mathrm{H}), 0.76(\mathrm{t}, J=7.1 \mathrm{~Hz}, 3 \mathrm{H})$.

${ }^{13} \mathrm{C}$ NMR (101 MHz, $\left.\mathrm{C}_{6} \mathrm{D}_{6}, 70{ }^{\circ} \mathrm{C}, \delta\right): 189.6,167.6,154.7,140.1,139.7,135.0,134.1,132.2$, $130.2,129.8(\mathrm{q}, J=33.4 \mathrm{~Hz}), 129.7(\mathrm{q}, J=30.5 \mathrm{~Hz}), 128.7,128.6,128.5$ (q, $J=2.9 \mathrm{~Hz}), 123.9$, 123.8 (q, $J=274.3 \mathrm{~Hz}), 123.7$ (q, $J=272.4 \mathrm{~Hz}), 123.0$ (hept, $J=4.7 \mathrm{~Hz}), 122.1,79.9,66.8$, 61.9, 35.8, $27.9(3 \mathrm{C}), 13.3$.

${ }^{19}$ F NMR $\left(376 \mathrm{MHz}, \mathrm{C}_{6} \mathrm{D}_{6}, \delta\right):-61.2,-62.8$.

HRMS (ESI): Calculated for $\mathrm{C}_{27} \mathrm{H}_{25} \mathrm{~F}_{6} \mathrm{NO}_{5} \mathrm{Na}(\mathrm{M}+\mathrm{Na})^{+} 580.1535$, found 580.1528 .

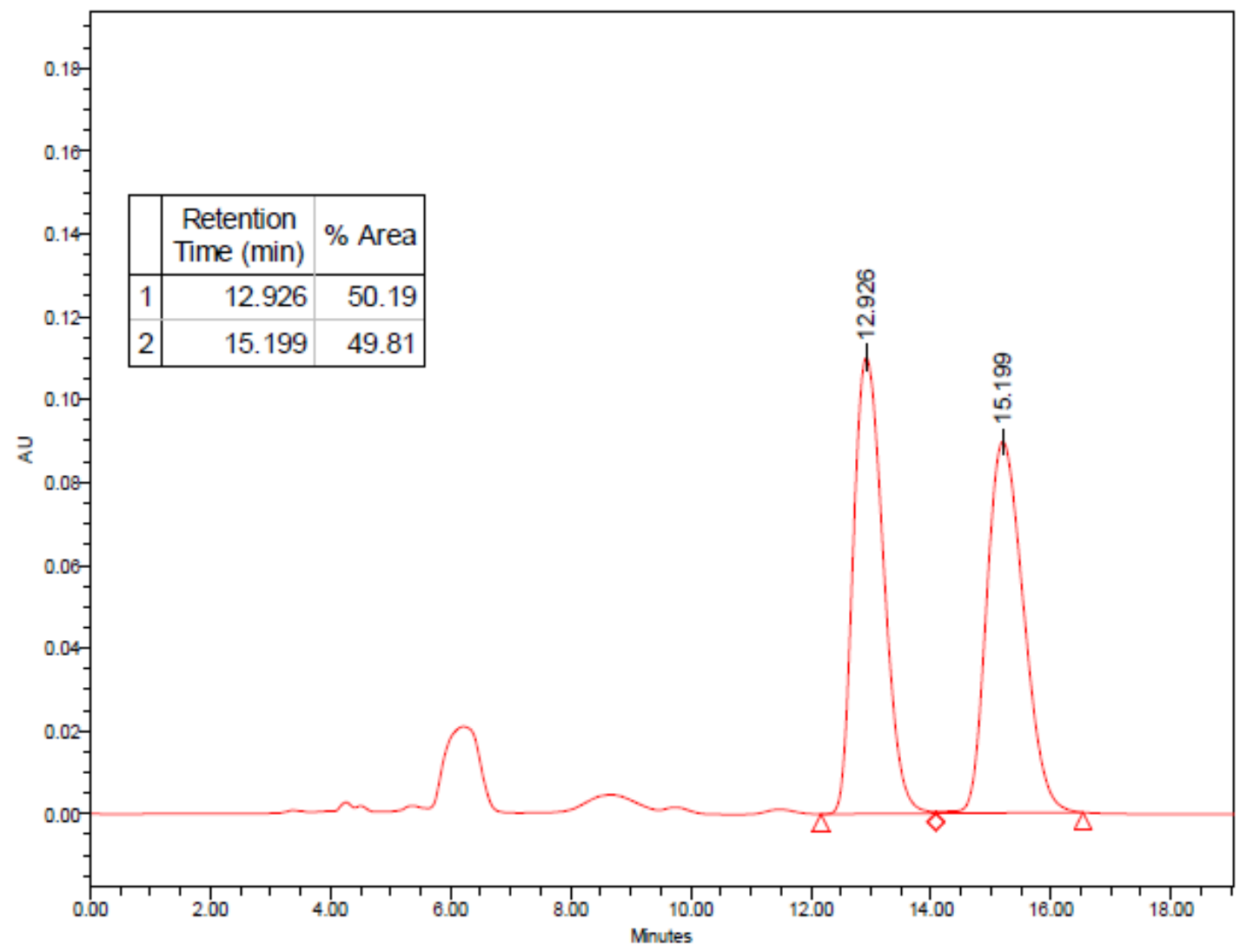




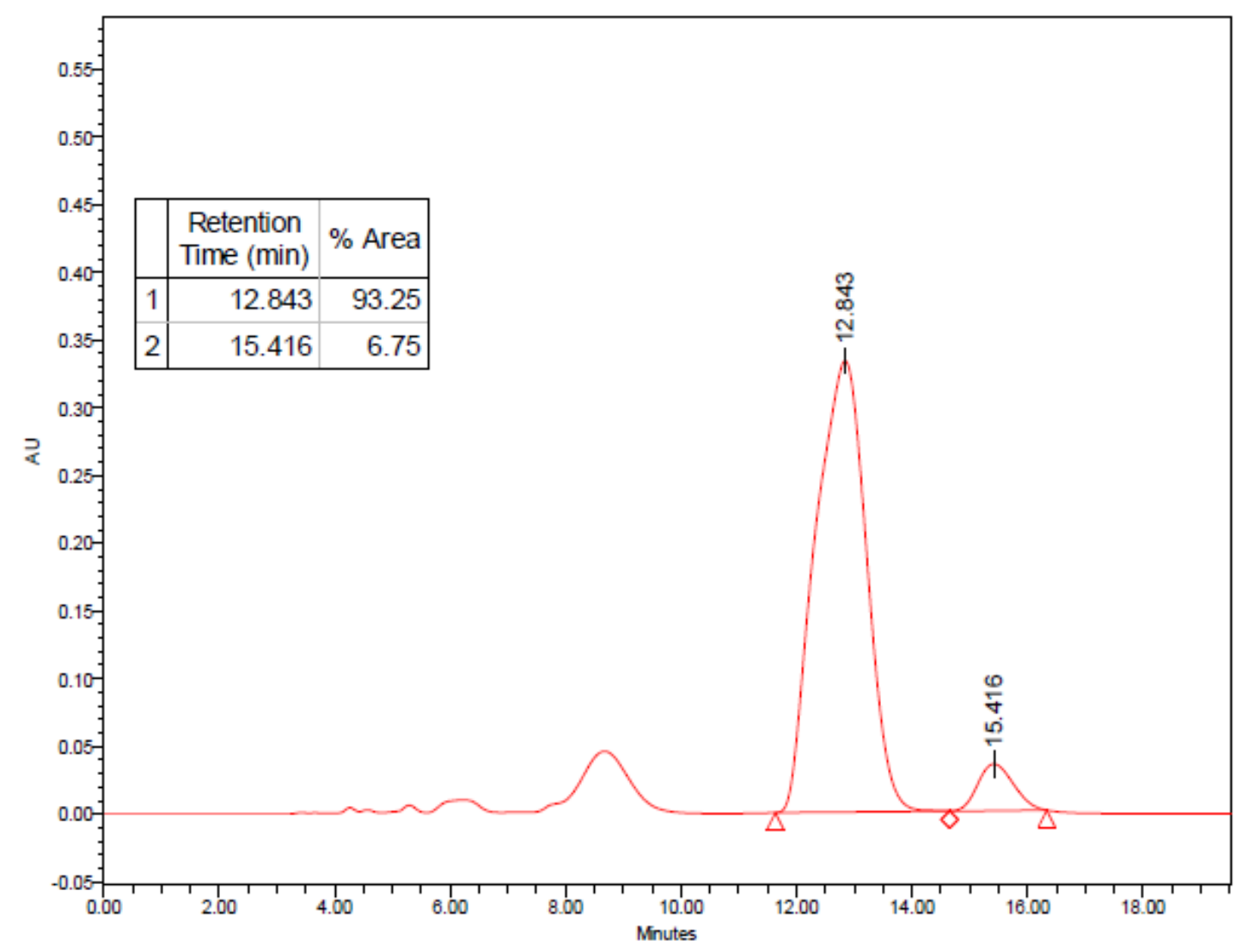

\section{Post-functionalization}

Ethyl

(S)-2-acetamido-6-methoxy-1,4-dioxo-1,2,3,4-tetrahydronaphthalene-2carboxylate (3p)<smiles>CCOC(=O)N[C@@]1(C(=O)OCC)C/C(=C\c2ccc(C(F)(F)F)cc2C(F)(F)F)c2cc(OC)ccc2C1=O</smiles>

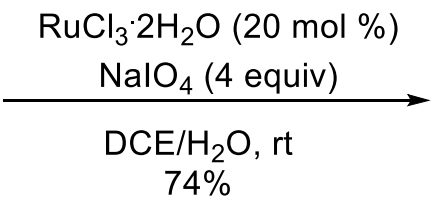<smiles>CCOC[C@]1(N)CC(=O)c2cc(OC)ccc2C1=O</smiles>

To a suspension of $\mathbf{2 p}(0.10 \mathrm{mmol}, 53 \mathrm{mg})$ and $\mathrm{RuCl}_{3} \cdot 2 \mathrm{H}_{2} \mathrm{O}(20 \mu \mathrm{mol}, 4.9 \mathrm{mg})$ in dichloroethane $(1 \mathrm{~mL})$ and water $(0.75 \mathrm{~mL})$ was added $\mathrm{NaIO}_{4}(0.40 \mathrm{mmol}, 86 \mathrm{mg})$ and the mixture was stirred vigorously. After total consumption of the starting material, monitored by TLC, the reaction was quenched with saturated aqueous solution of $\mathrm{Na}_{2} \mathrm{~S}_{2} \mathrm{O}_{3}(2 \mathrm{~mL}) \cdot \mathrm{CH}_{2} \mathrm{Cl}_{2}(5 \mathrm{~mL})$ was added. The two layers were separated and the aqueous phase was extracted with $\mathrm{CH}_{2} \mathrm{Cl}_{2}$. The combined organic layers were washed with brine, dried over $\mathrm{MgSO}_{4}$, filtered and concentrated under vacuum. The residue was purified by flash chromatography on silica gel (petroleum ether/EtOAc 50:50) to afford 3p as yellow oil (24 mg, $74 \%)$.

$\mathbf{R}_{\mathbf{f}}=0.09$ (petroleum ether/EtOAc 6:4).

HPLC (Chiralpak ID hexane $/ \mathrm{iPrOH}$ 30:70, $1.0 \mathrm{~mL} / \mathrm{min}$ ): $\mathrm{Rt}=13.8 \mathrm{~min}$ (major), $31.9 \mathrm{~min}$ (minor).

$[\alpha]_{\mathbf{D}^{20}}=+68\left(\mathrm{c}=0.9, \mathrm{CHCl}_{3}\right)$ for an enantiomeric excess of $91 \%$.

${ }^{1} \mathbf{H}$ NMR $\left(400 \mathrm{MHz}, \mathrm{CDCl}_{3}, \delta\right): 8.03(\mathrm{~d}, J=8.7 \mathrm{~Hz}, 1 \mathrm{H}), 7.56(\mathrm{~d}, J=2.7 \mathrm{~Hz}, 1 \mathrm{H}), 7.25-7.13$ $(\mathrm{m}, 2 \mathrm{H}), 4.16-4.03(\mathrm{~m}, 2 \mathrm{H}), 3.96(\mathrm{~s}, 3 \mathrm{H}), 3.94(\mathrm{~d}, J=16.4 \mathrm{~Hz}, 1 \mathrm{H}), 3.22(\mathrm{~d}, J=16.4 \mathrm{~Hz}, 1 \mathrm{H})$, $2.11(\mathrm{~s}, 3 \mathrm{H}), 1.08(\mathrm{t}, J=7.1 \mathrm{~Hz}, 3 \mathrm{H})$. 
${ }^{13}$ C NMR (101 MHz, $\left.\mathrm{CDCl}_{3}, \delta\right): 191.3,187.5,170.0,167.5,165.3,138.5,130.5,125.6,121.5$, $108.9,68.4,63.4,56.1,46.2,23.1,13.7$.

HRMS (ESI): Calculated for $\mathrm{C}_{16} \mathrm{H}_{18} \mathrm{NO}_{6}(\mathrm{M}+\mathrm{H})^{+} 320.1134$, found 320.1129 .
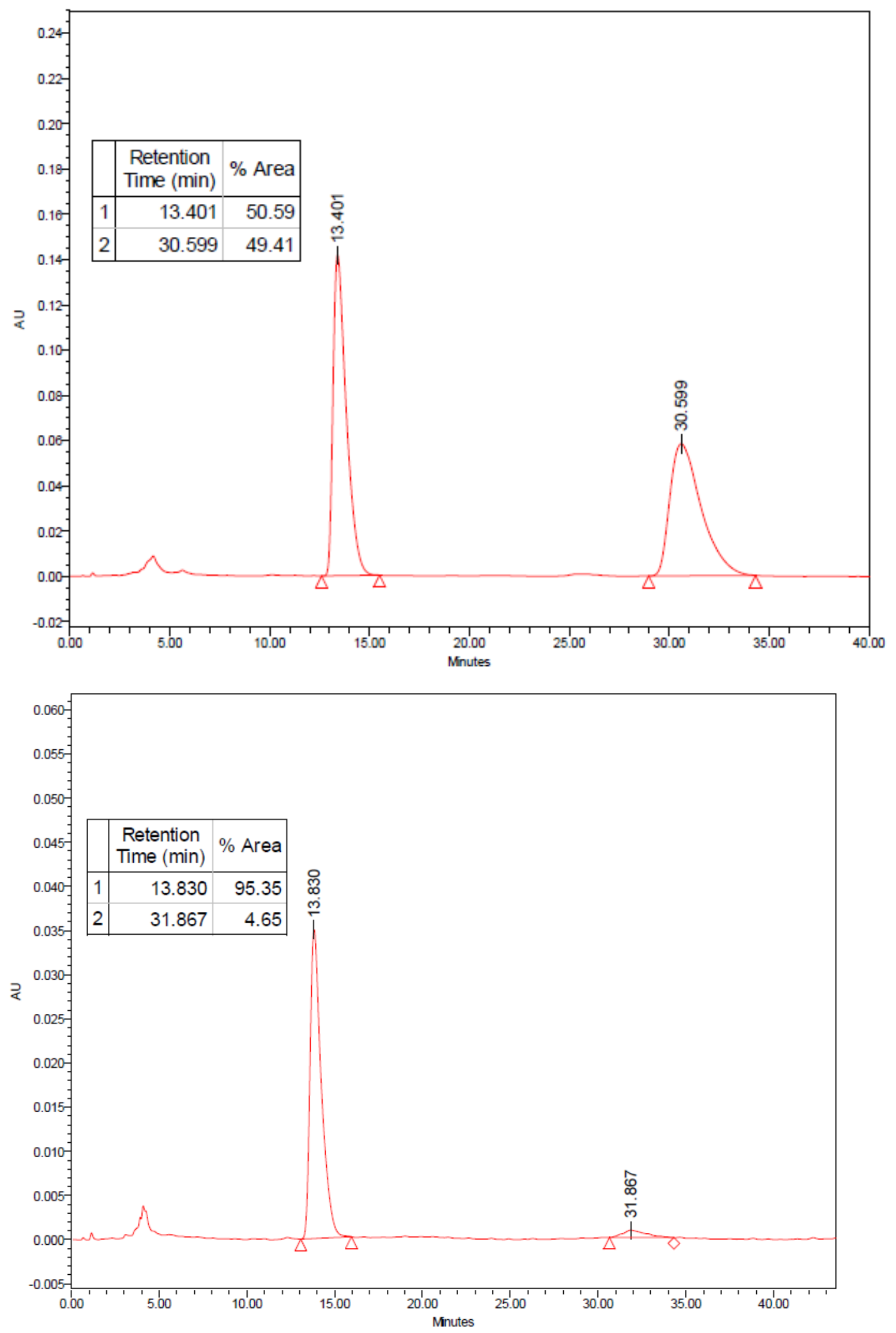


\section{X-ray diffraction of $\mathbf{2 j}$}

A single crystal of the compound was selected, mounted onto a cryoloop, and transferred in a cold nitrogen gas stream. Intensity data were collected with a BRUKER Kappa-APEXII diffractometer with a copper-K $\alpha$ microfocus source $(\lambda=1.54178 \AA)$. Data collection was performed with APEX2 suite (BRUKER). Unit-cell parameters refinement, integration and data reduction were carried out with SAINT program (BRUKER). SADABS (BRUKER) was used for scaling and multi-scan absorption corrections. In the WinGX suite of programs ${ }^{1}$, the structure was solved with SHELXT $-14^{2}$ program and refined by full-matrix least-squares methods using SHELXL-14².

Crystal data for $\mathbf{2 j}$. Colourless needles: $\mathrm{C}_{27} \mathrm{H}_{26} \mathrm{~F}_{6} \mathrm{O}_{4}$, monoclinic, $P 2_{1}, \mathrm{a}=14.2106(5), \mathrm{b}=$ 11.4907(3), $\mathrm{c}=17.0087(6) \AA, \mathrm{V}=2541.55(15) \AA^{3}, \mathrm{Z}=4, \mathrm{~T}=200(1) \mathrm{K}, \mu=1.046 \mathrm{~mm}^{-1}, 8906$ reflections measured, 2205 independent $\left(\mathrm{R}_{\text {int }}=0.0171\right), 2109$ observed $[\mathrm{I} \geq 2 \sigma$ (I)], 676 parameters, final $\mathrm{R}$ indices $\mathrm{R}_{1}[\mathrm{I} \geq 2 \sigma(\mathrm{I})]=0.0270$ and $\mathrm{wR}_{2}$ (all data) $=0.1032$, GOF on $\mathrm{F}^{2}=$ $1.029, \mathrm{max} / \mathrm{min}$ residual electron density $=0.33 /-0.23 \mathrm{e} . \AA^{-3}$.

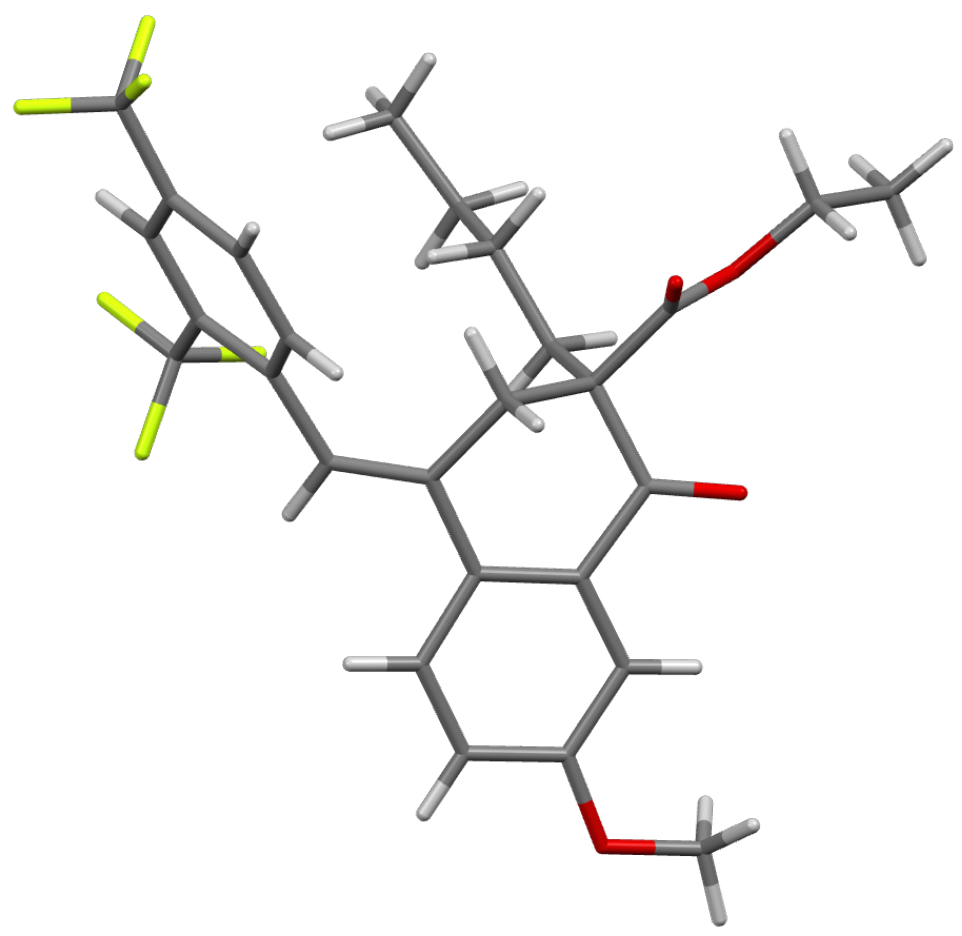

\footnotetext{
${ }^{1}$ Farrugia, L. J., J. Appl. Crystallogr. 1999, 32, 837.

${ }^{2}$ Palatinus, L.; Chapuis, G., J. Appl. Crystallogr. 2007, 40, 786.

${ }^{3}$ Sheldrick, G. M., Acta Crystallogr. C Struct. Chem. 2015, 71, 3.
} 


\section{NMR spectra}
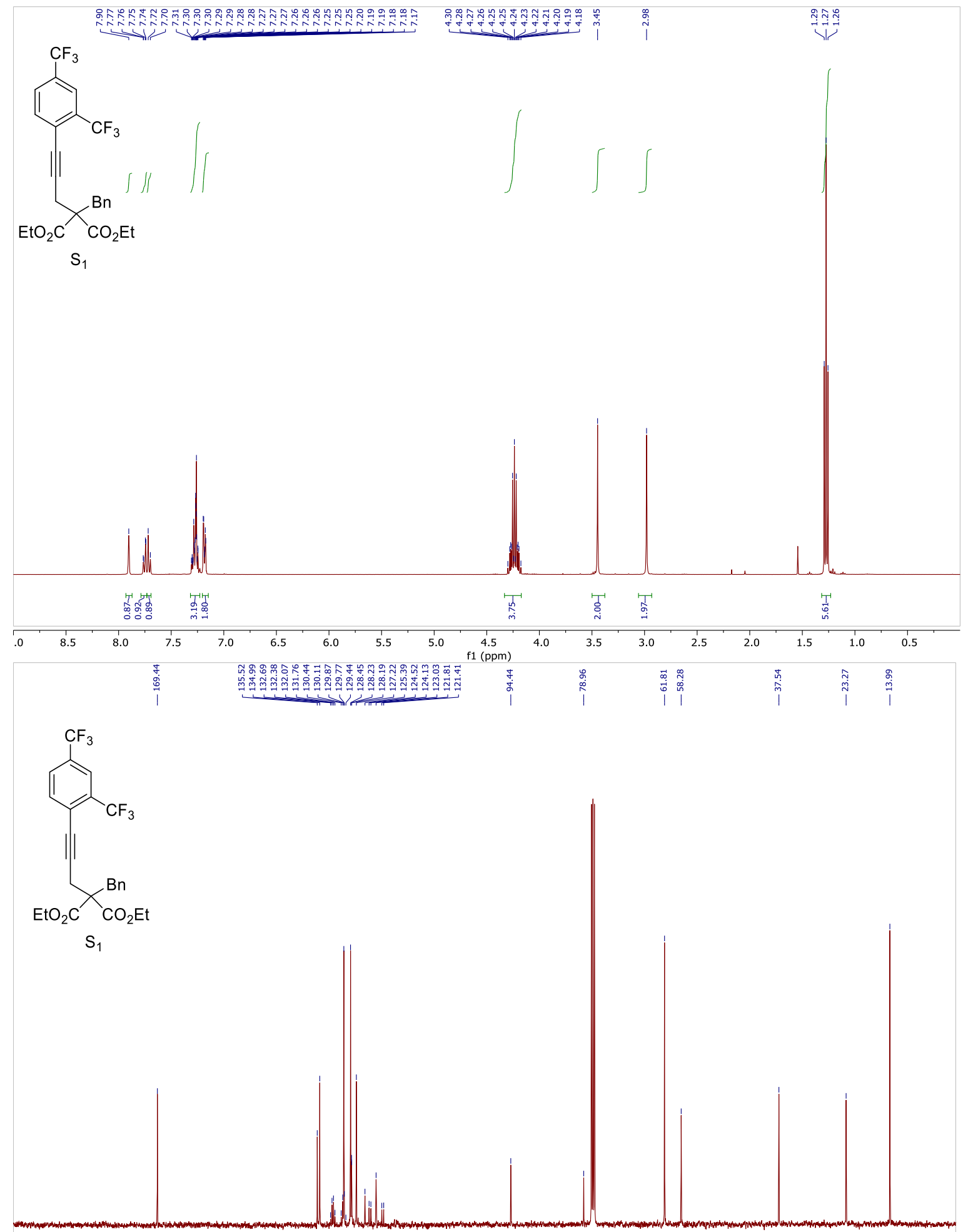

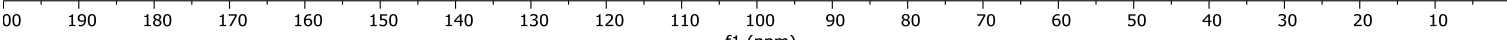




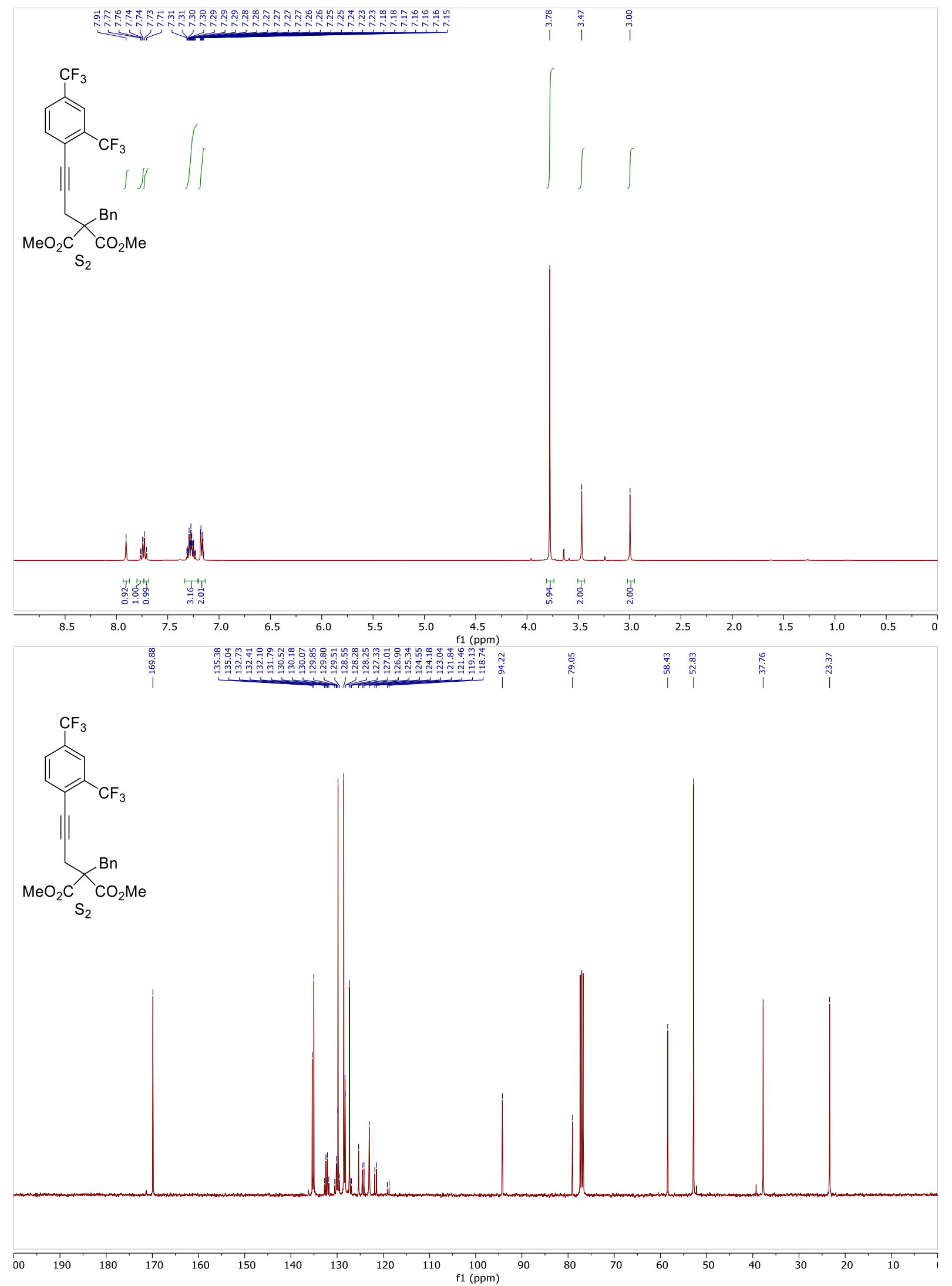




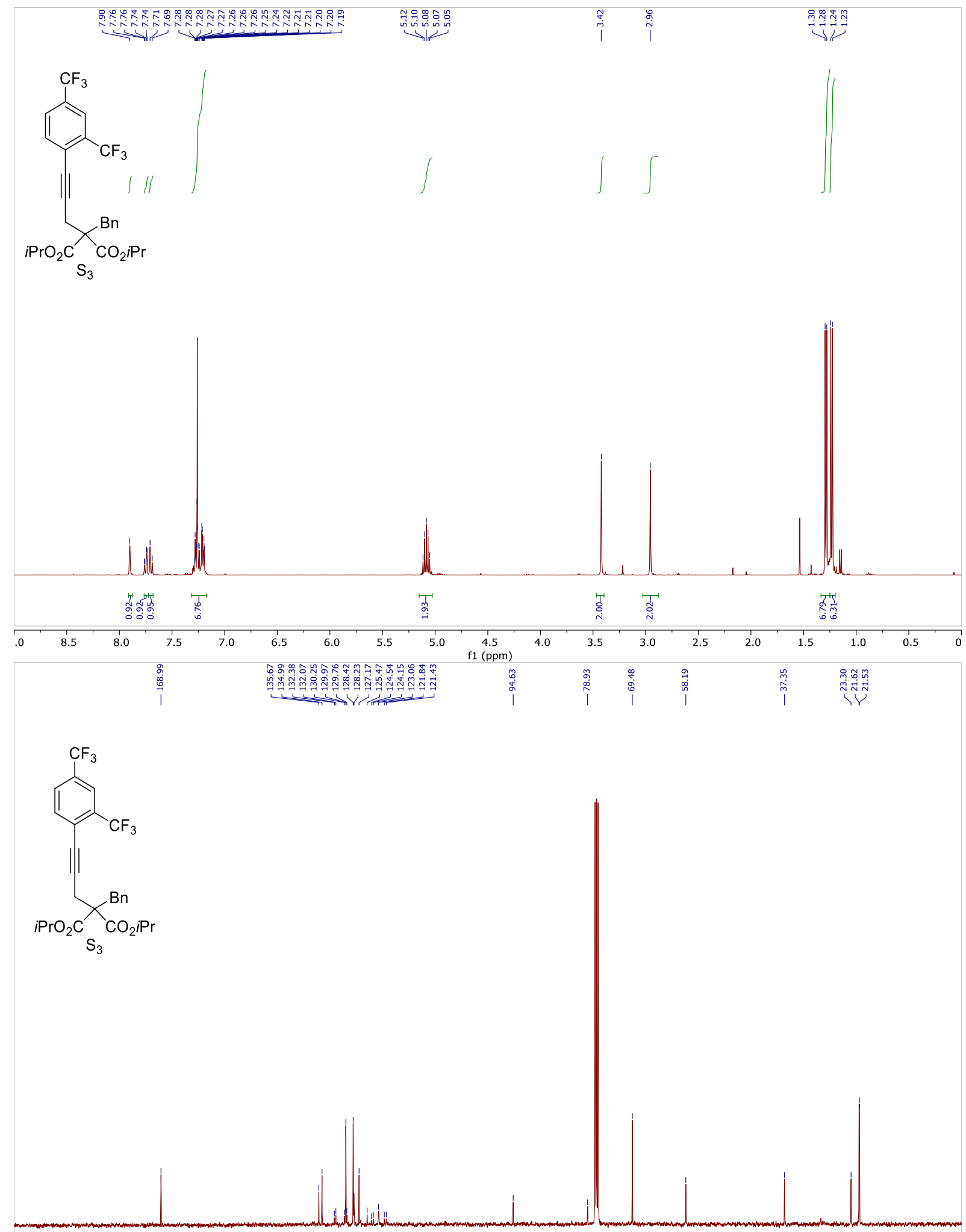

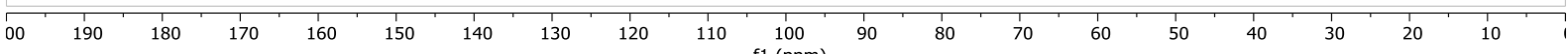



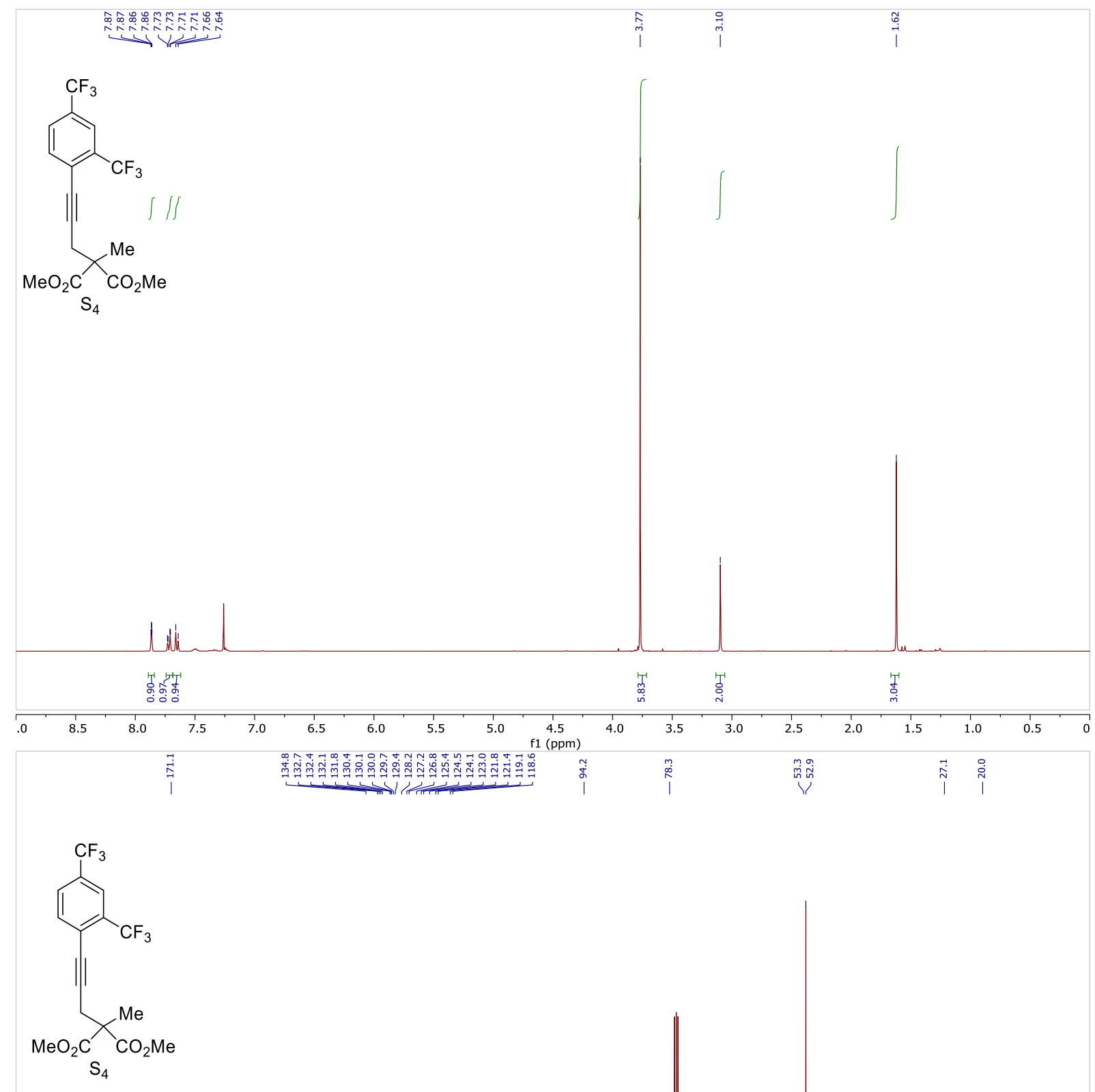


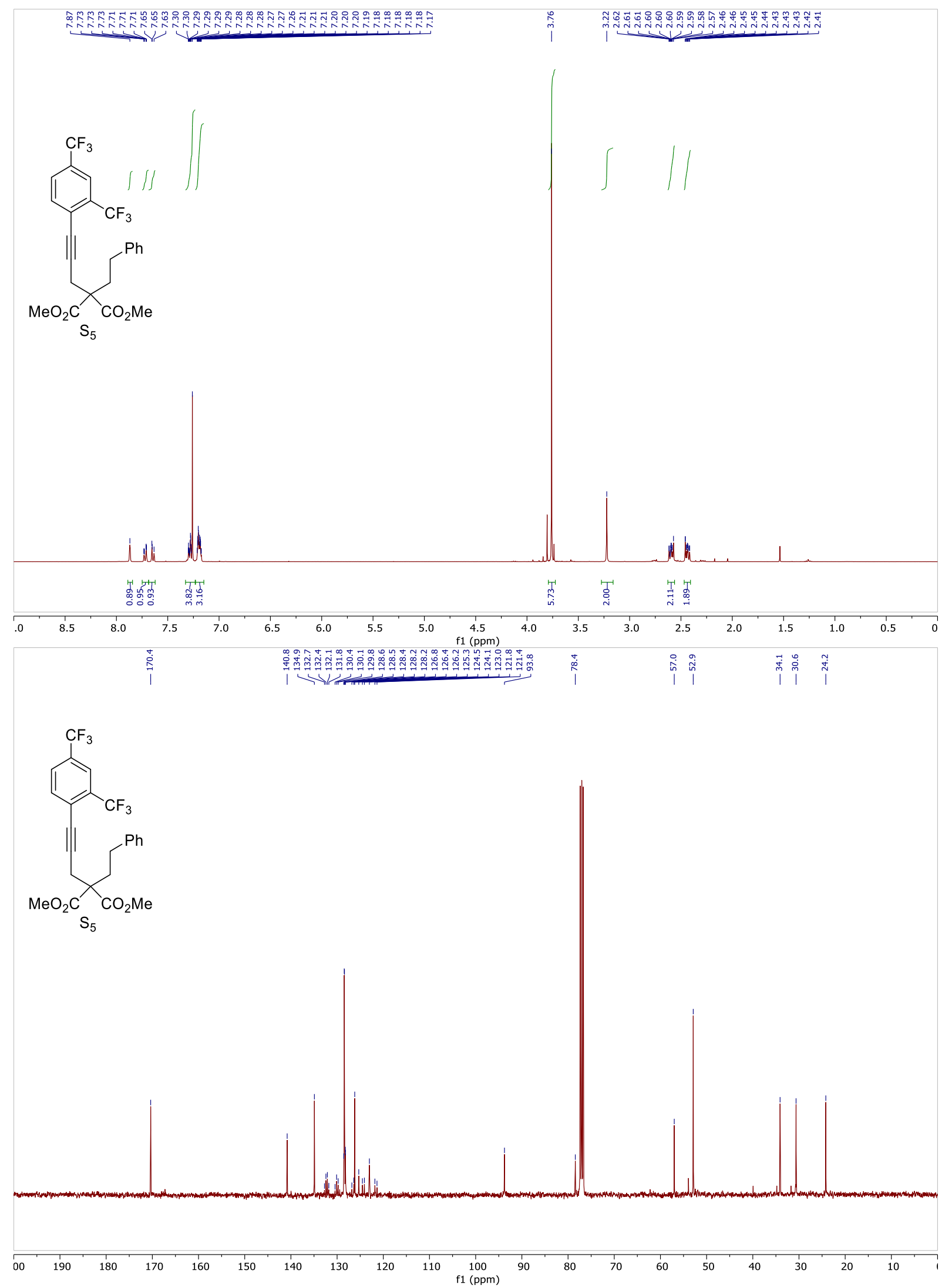




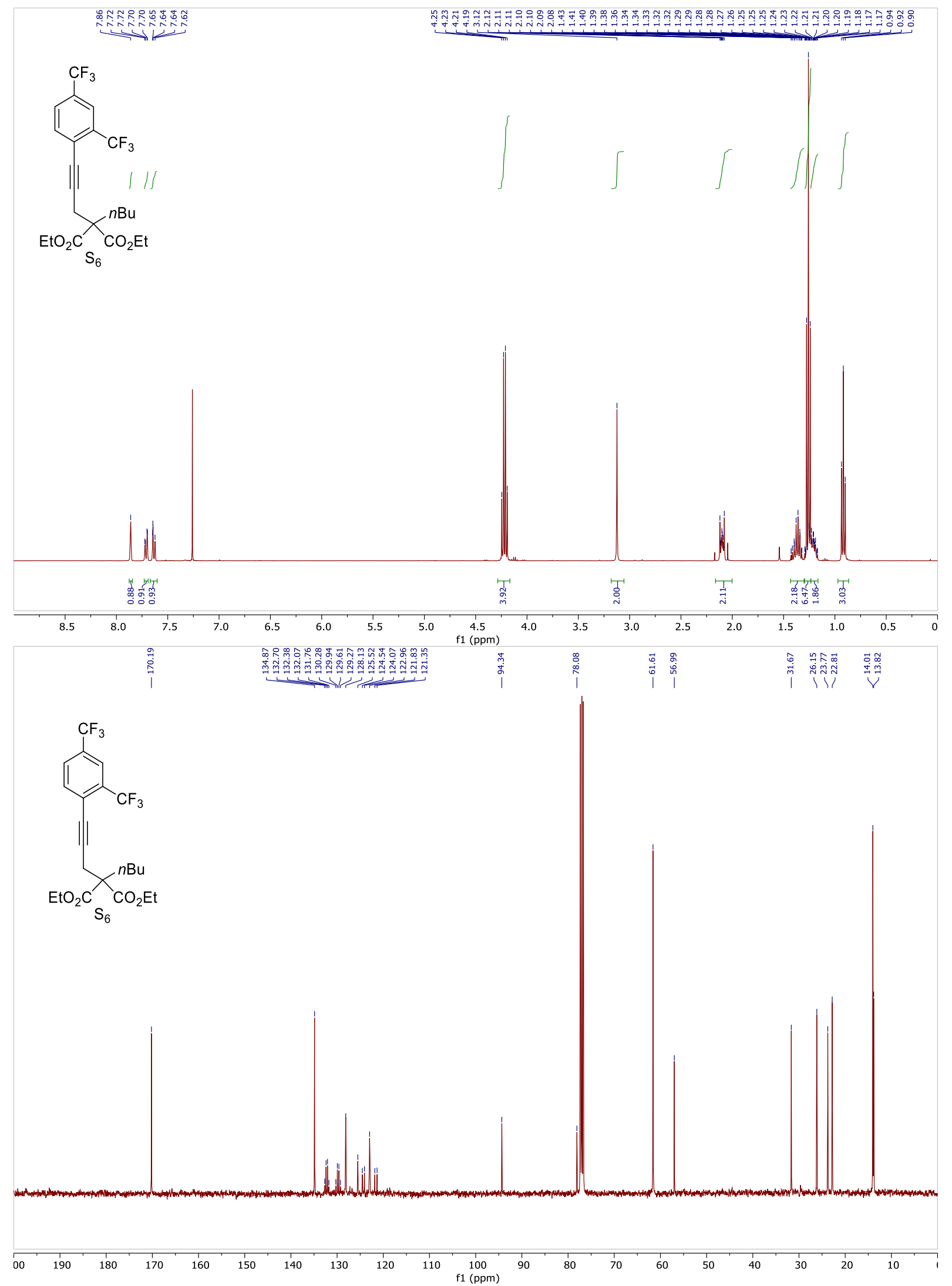




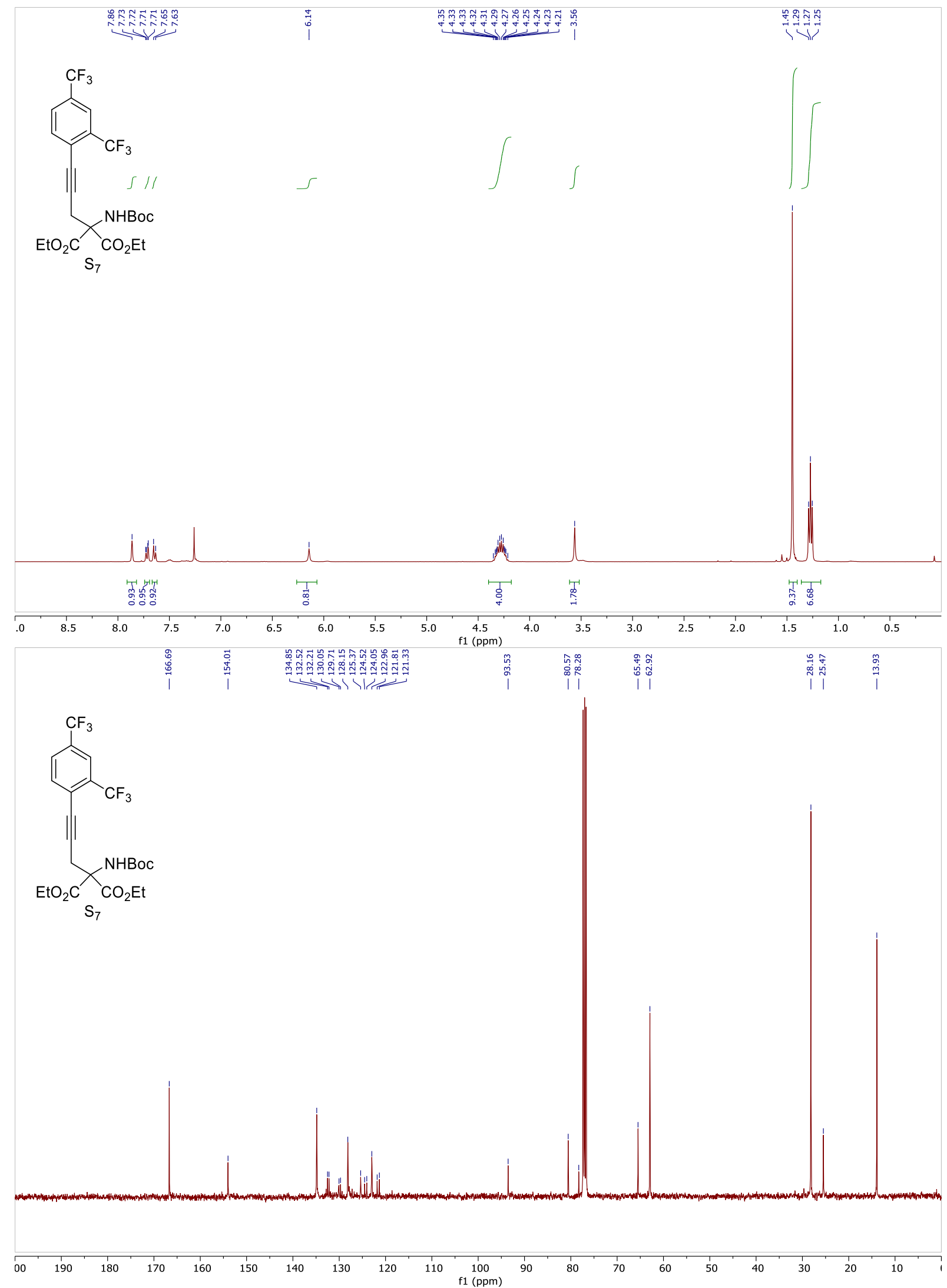




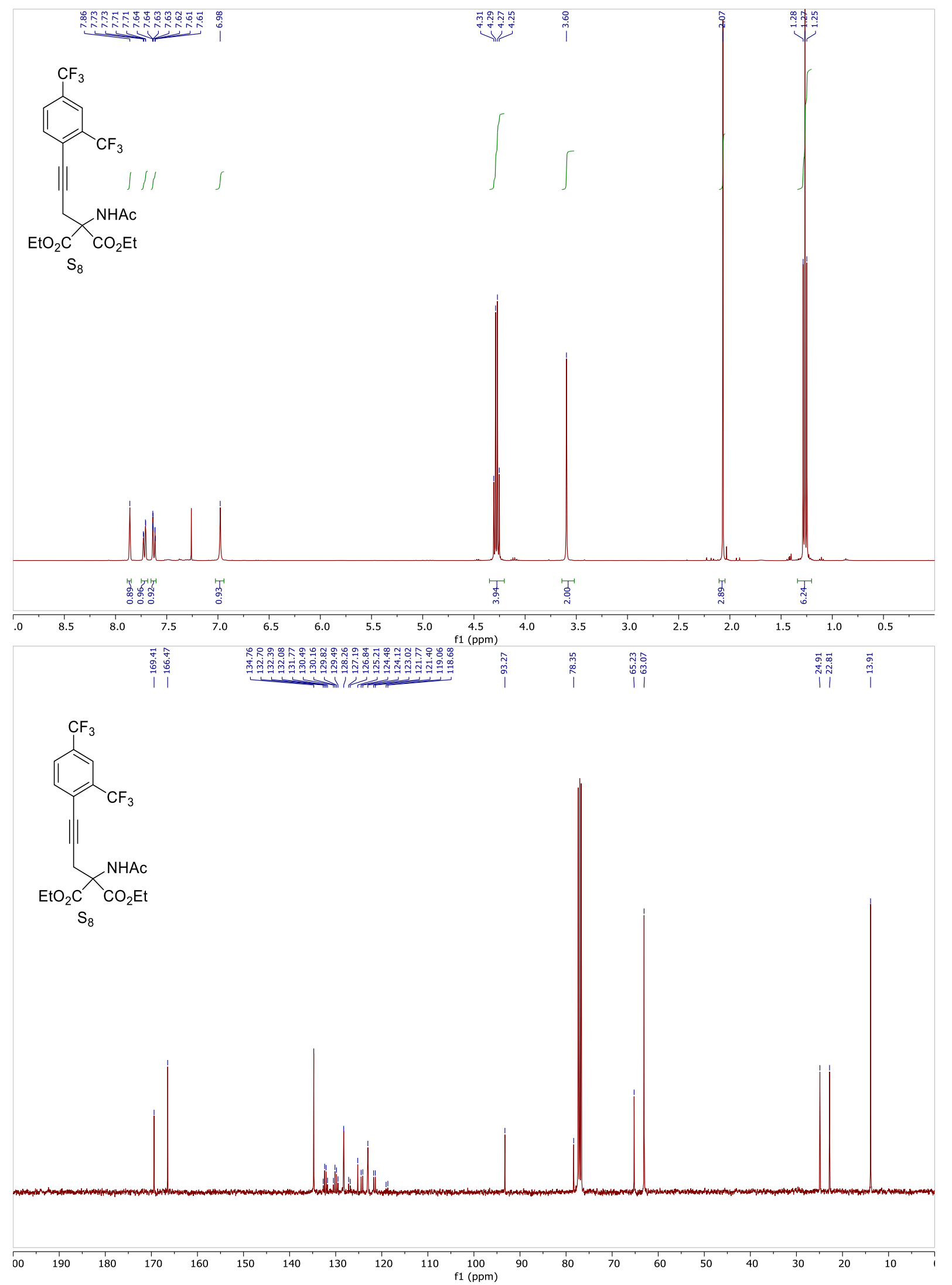




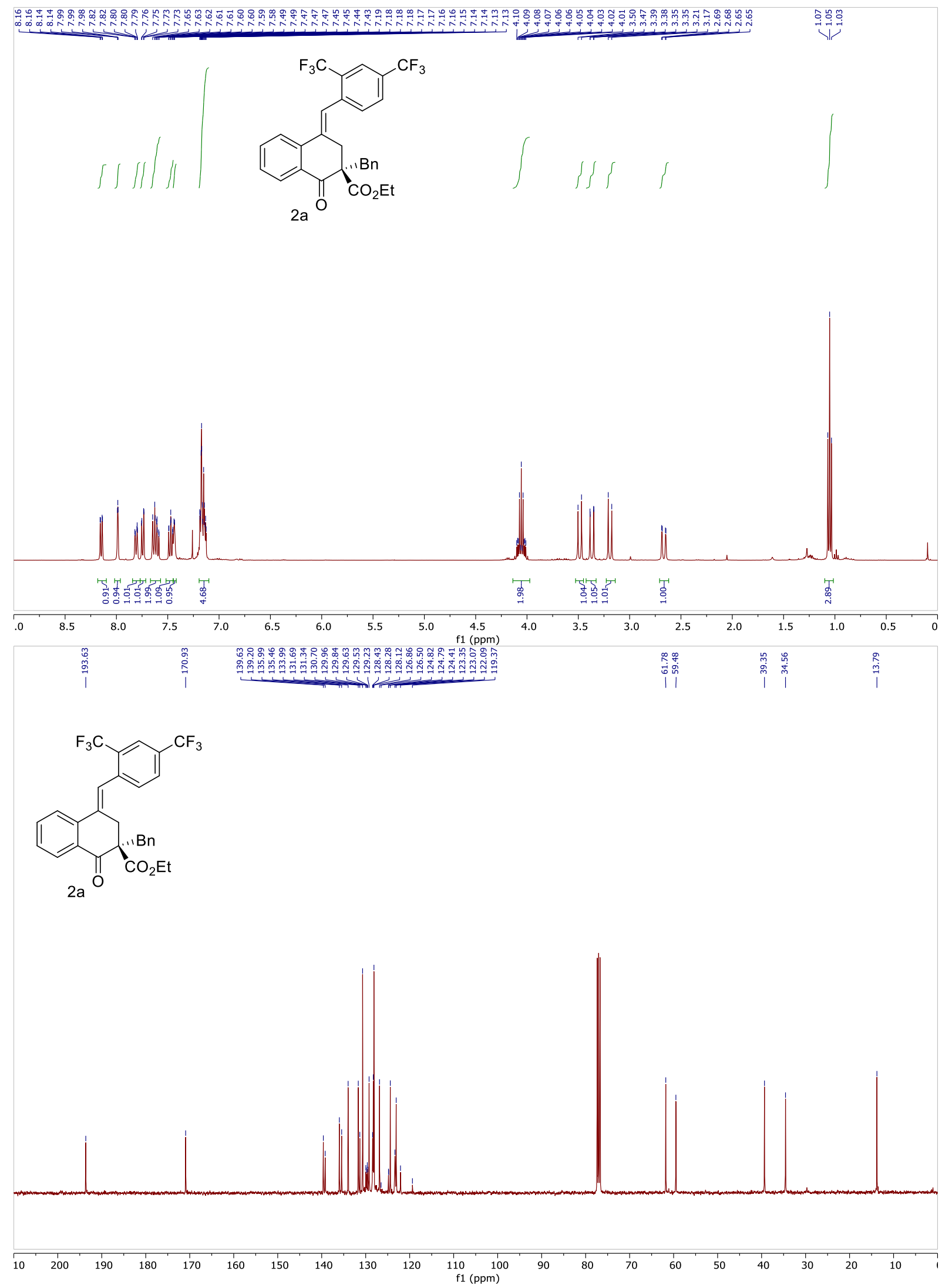



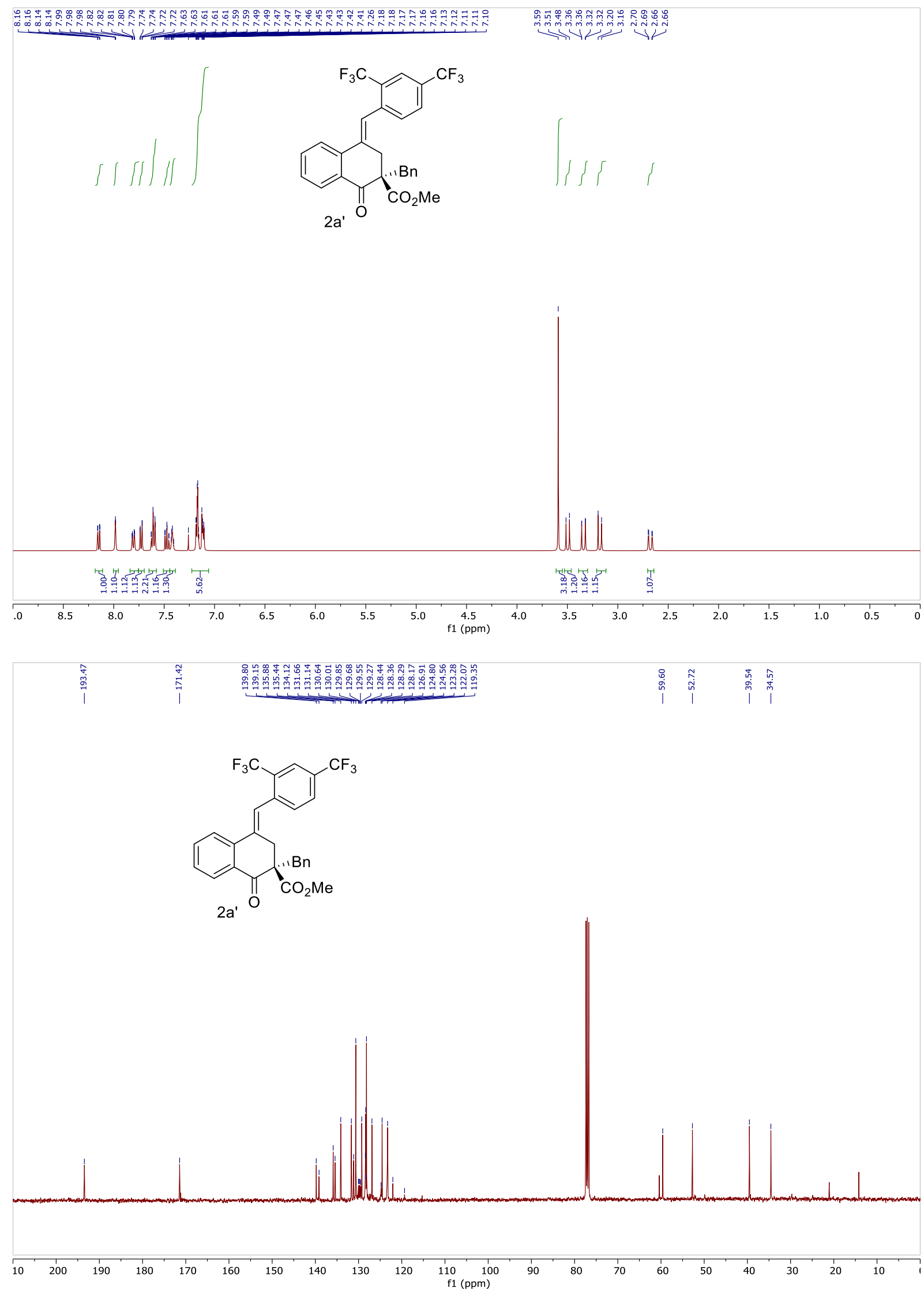


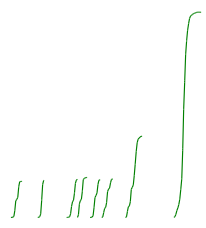<smiles>CC(C)OC(=O)C1(Cc2ccccc2)C/C(=C\c2ccc(C(F)(F)F)cc2C(F)(F)F)c2ccccc2C1=O</smiles>

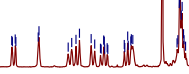
iti

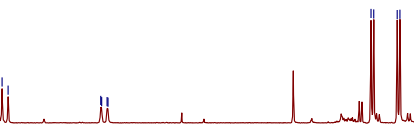

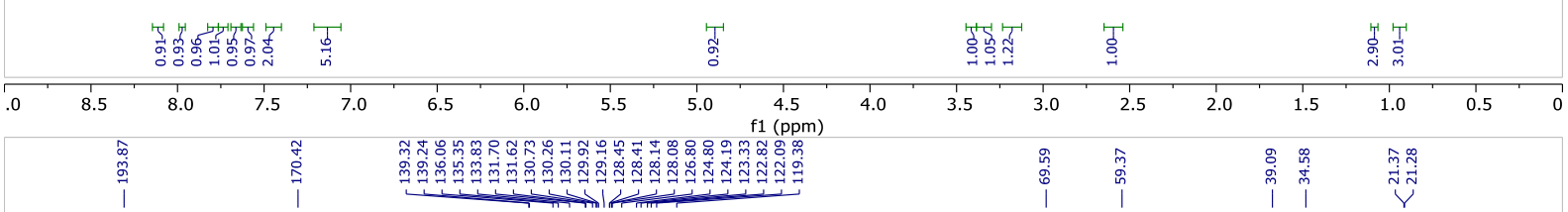<smiles>CCCOC(=O)[C@]1(Cc2ccccc2)C/C(=C\c2ccc(C(F)(F)F)cc2C(F)(F)F)c2ccccc2C1=O</smiles>

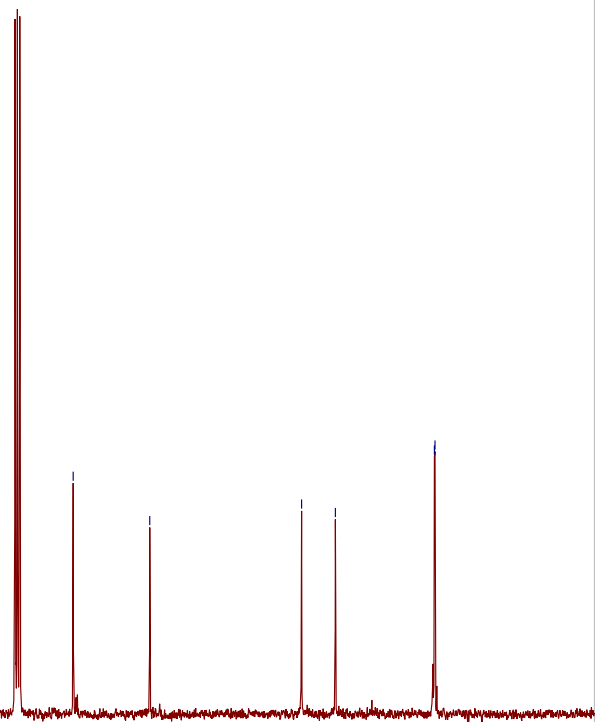

10 110
$\mathrm{f} 1(\mathrm{ppm})$

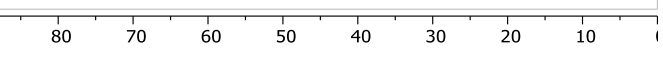




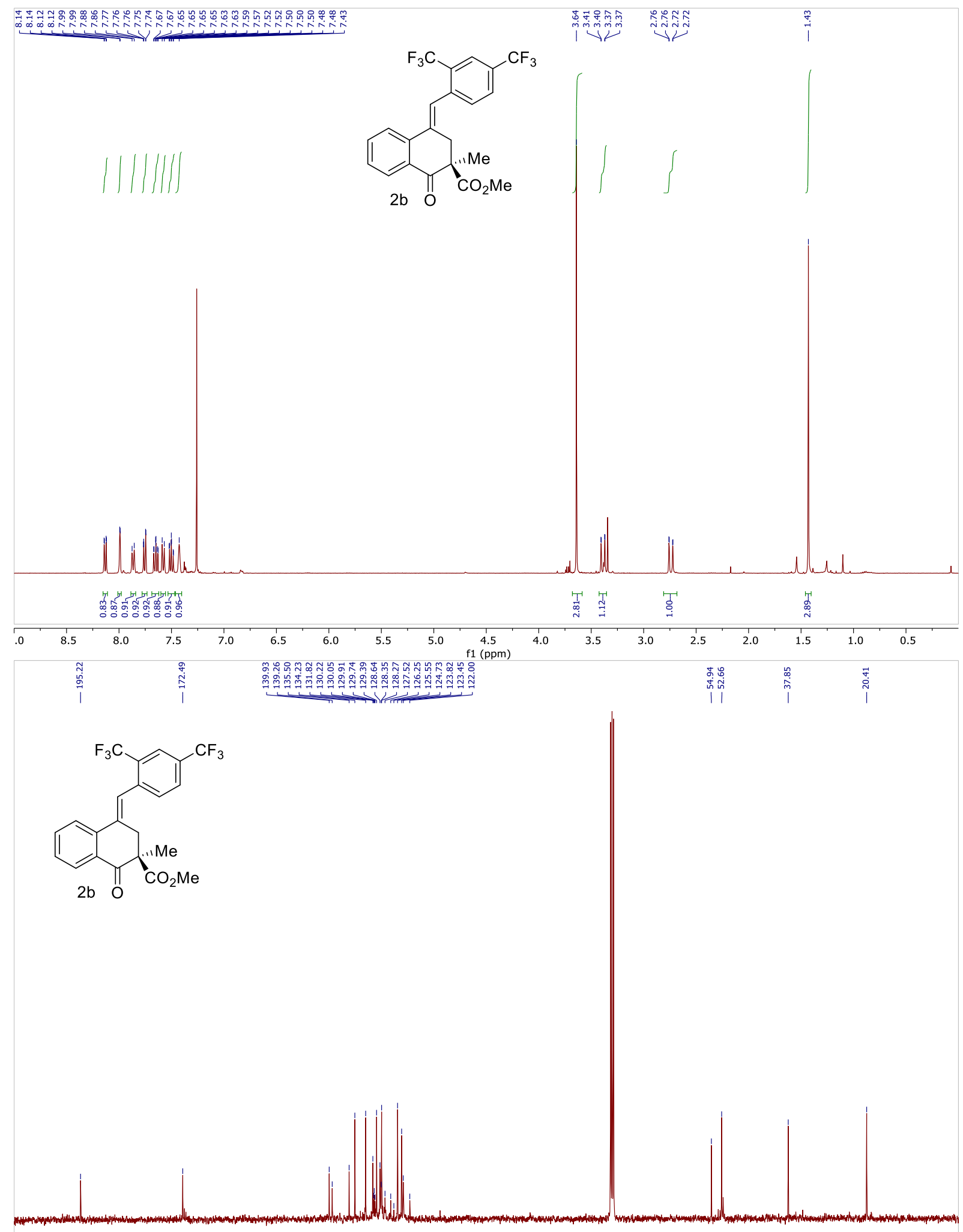

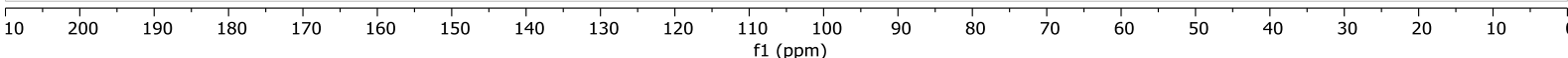




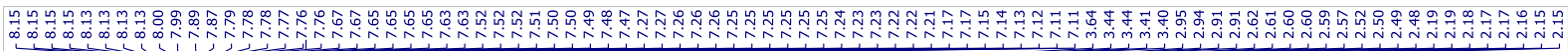

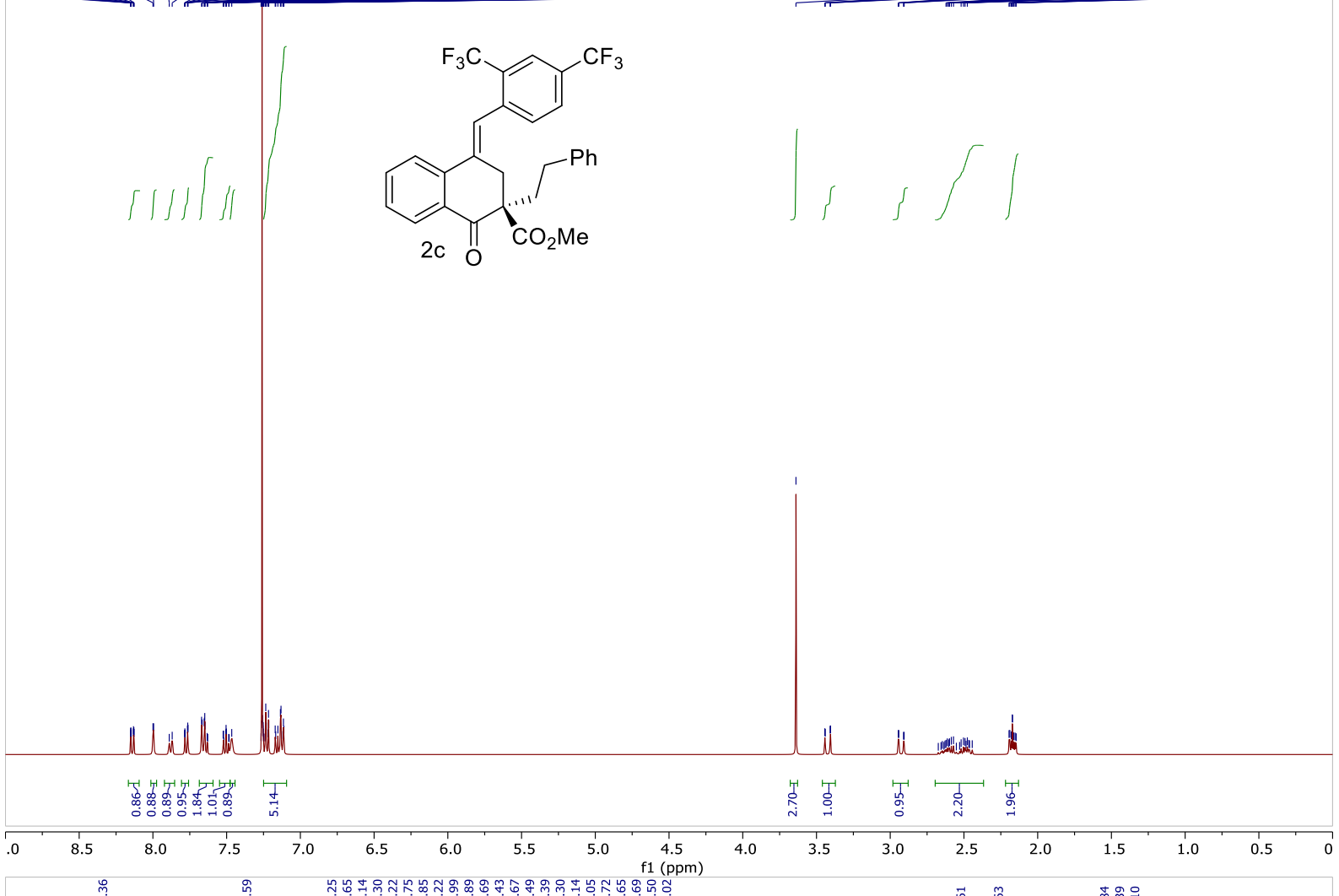

尊

总
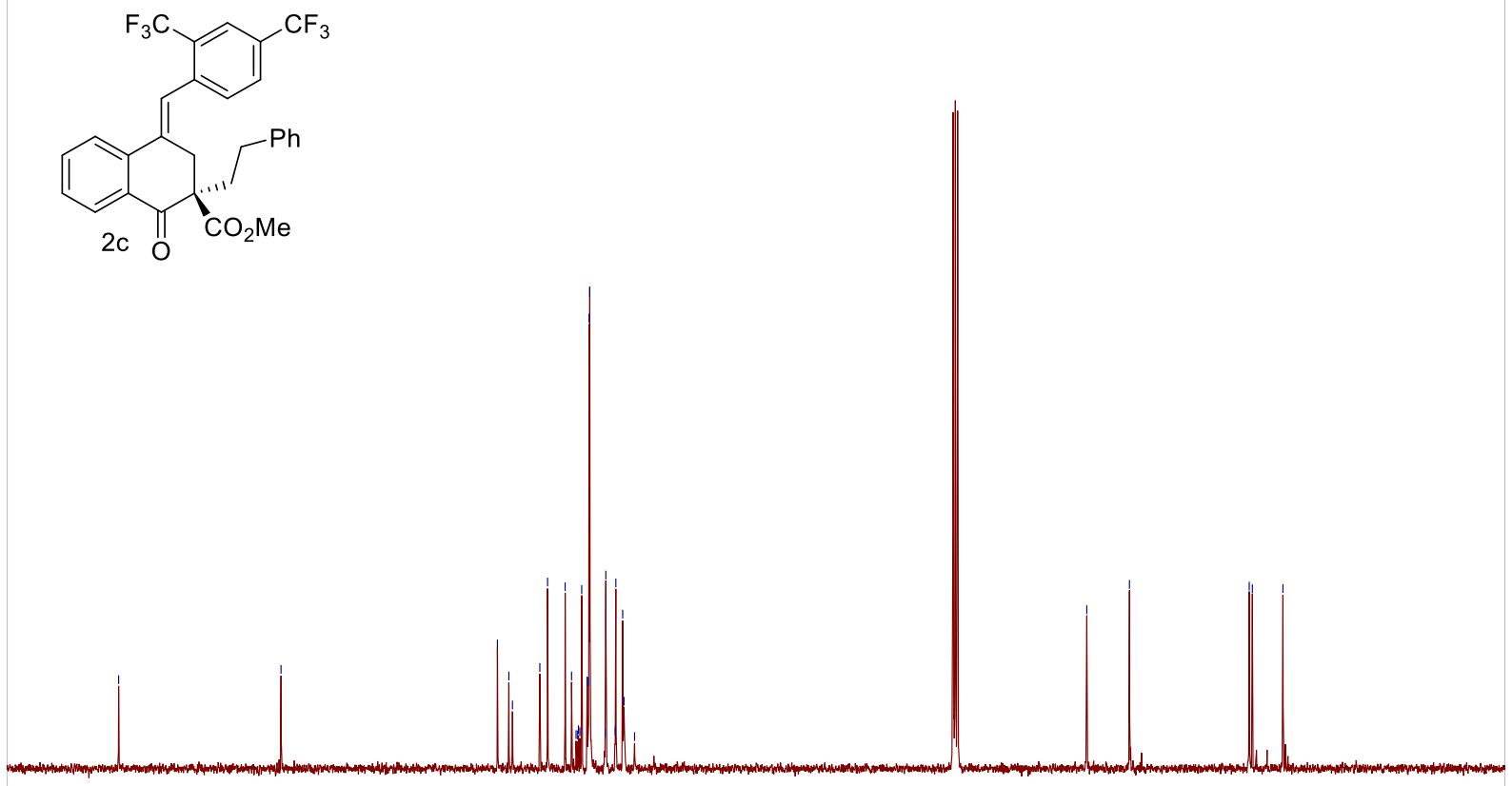

$\begin{array}{lllllllllllllllllllllll}10 & 200 & 190 & 180 & 170 & 160 & 150 & 140 & 130 & 120 & 110 & 100 & 90 & 80 & 70 & 60 & 50 & 40 & 30 & 20 & 10 & 10\end{array}$ 

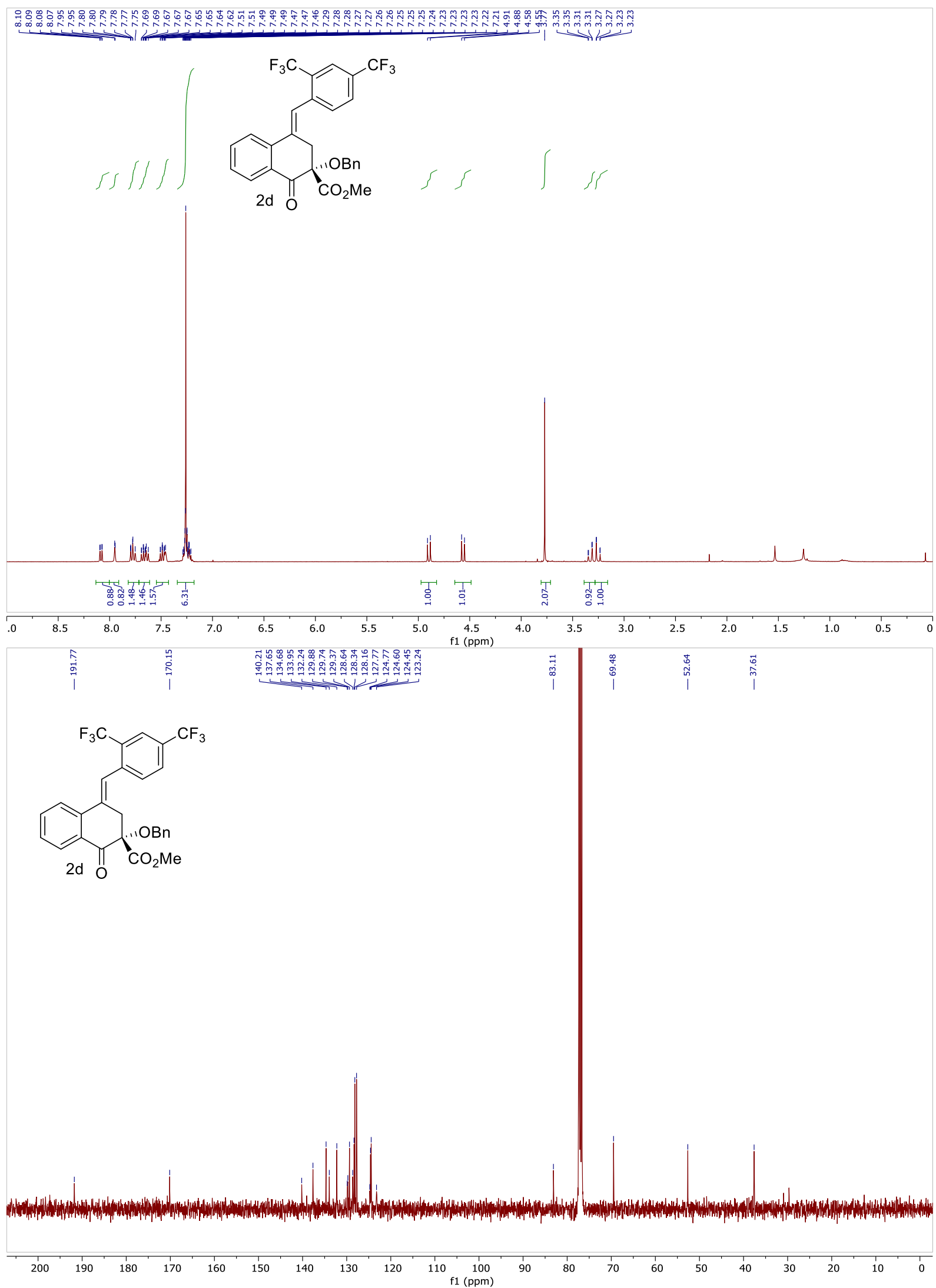


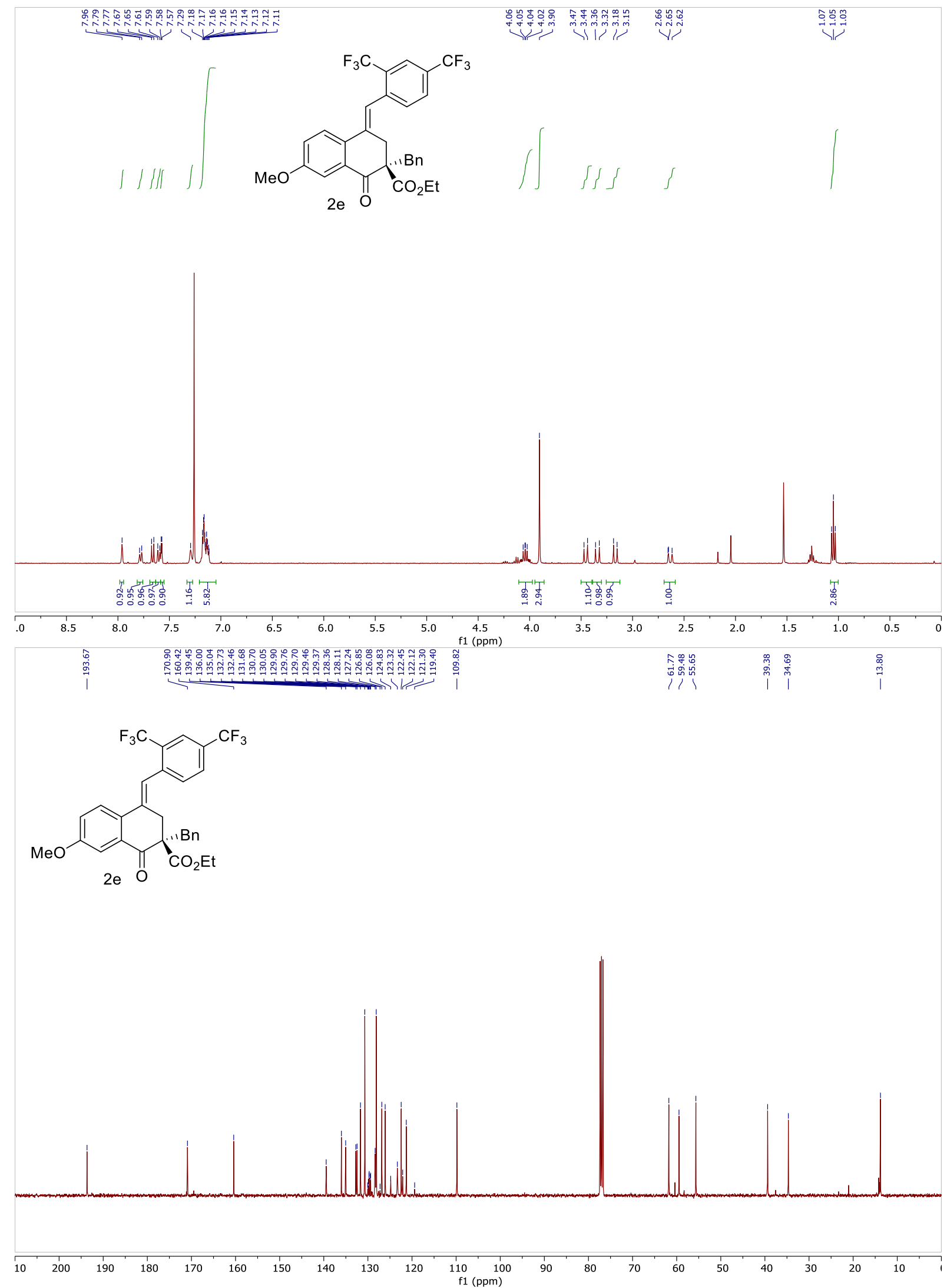




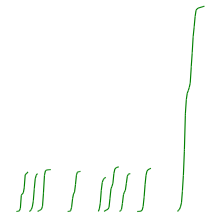<smiles>CCOC(=O)C1(CBr)CC(=Cc2ccc(C(F)(F)F)cc2C(F)(F)F)c2cc(NC(C)C)ccc2C1=O</smiles>
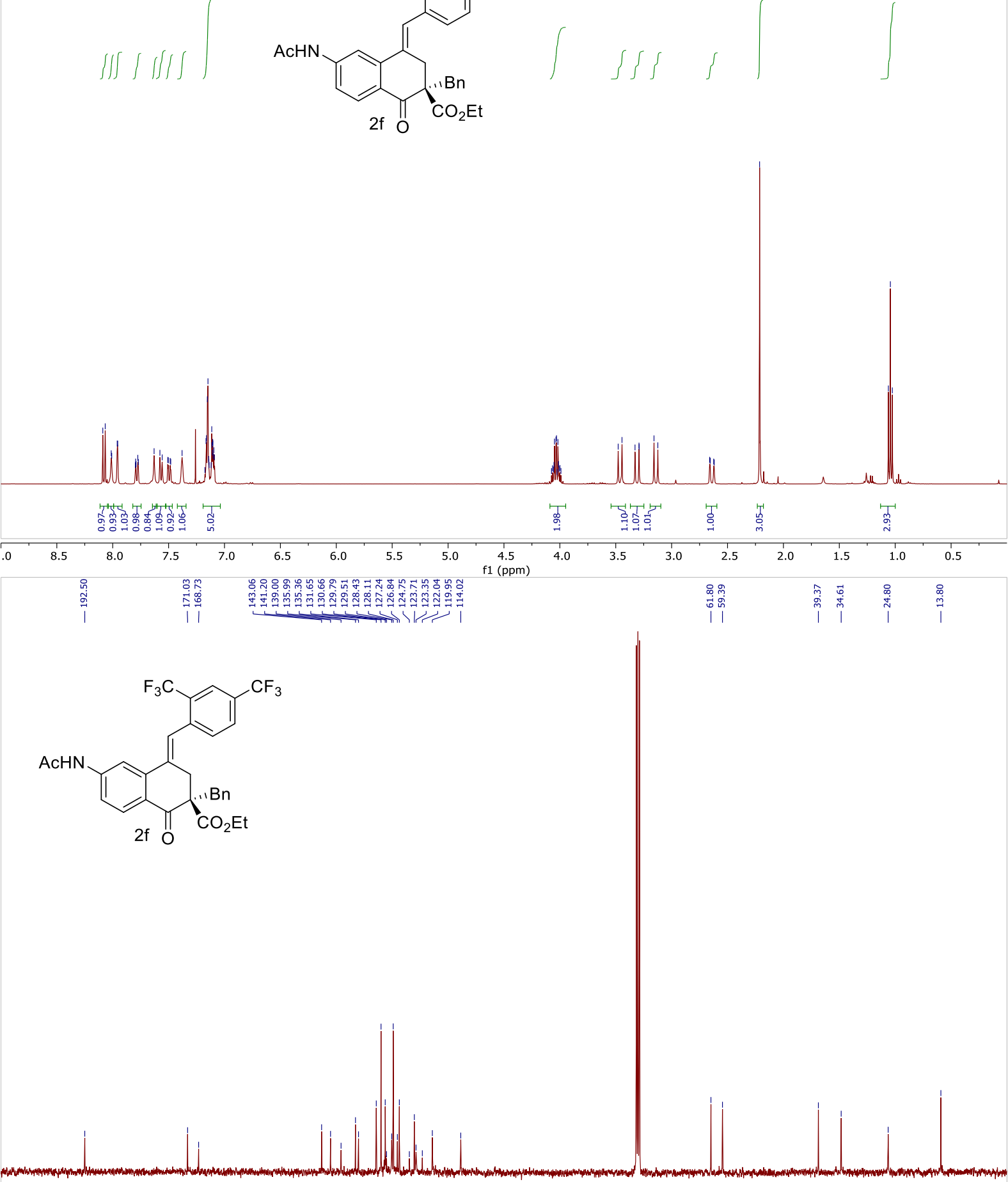

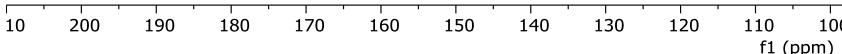




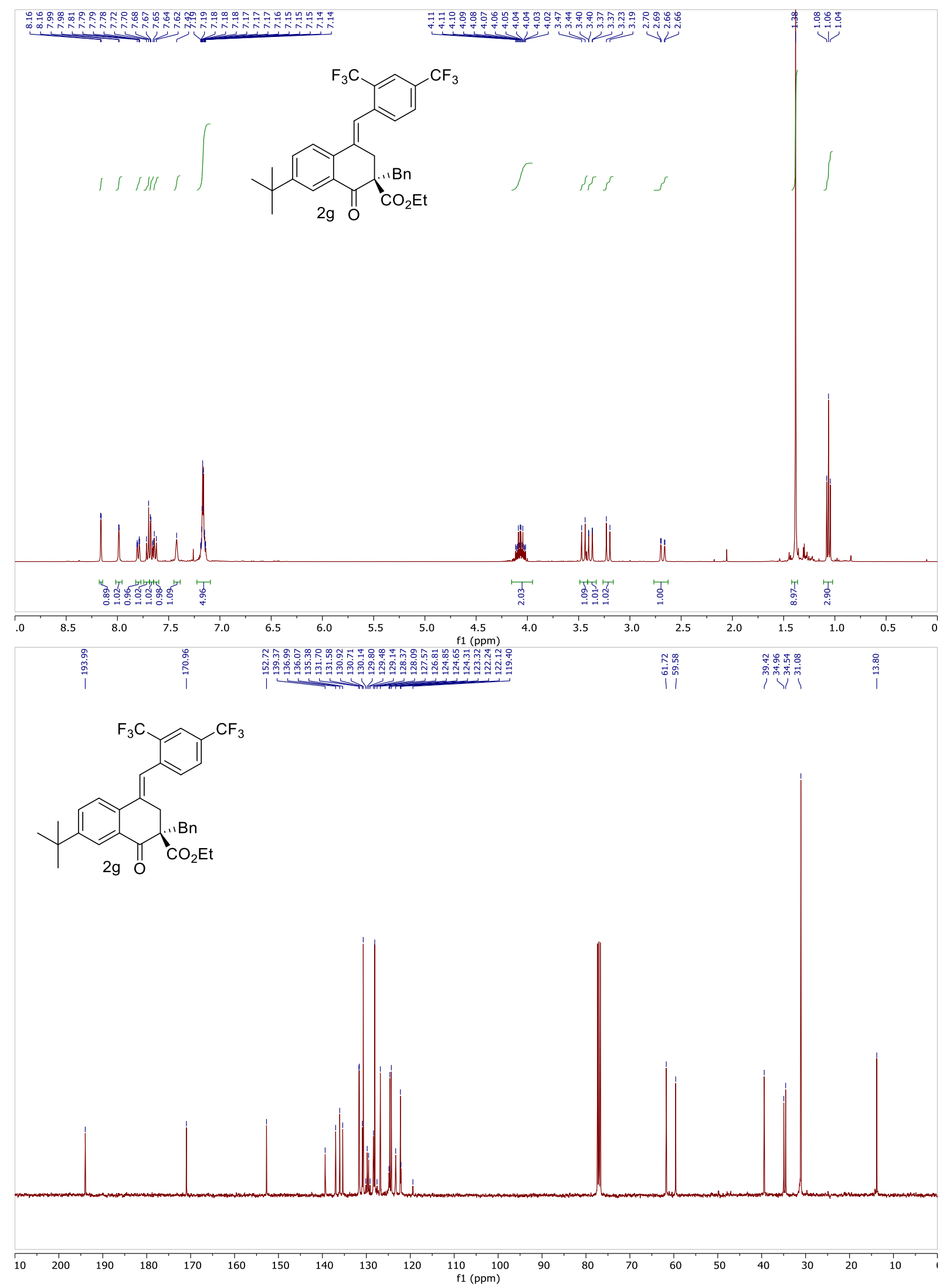




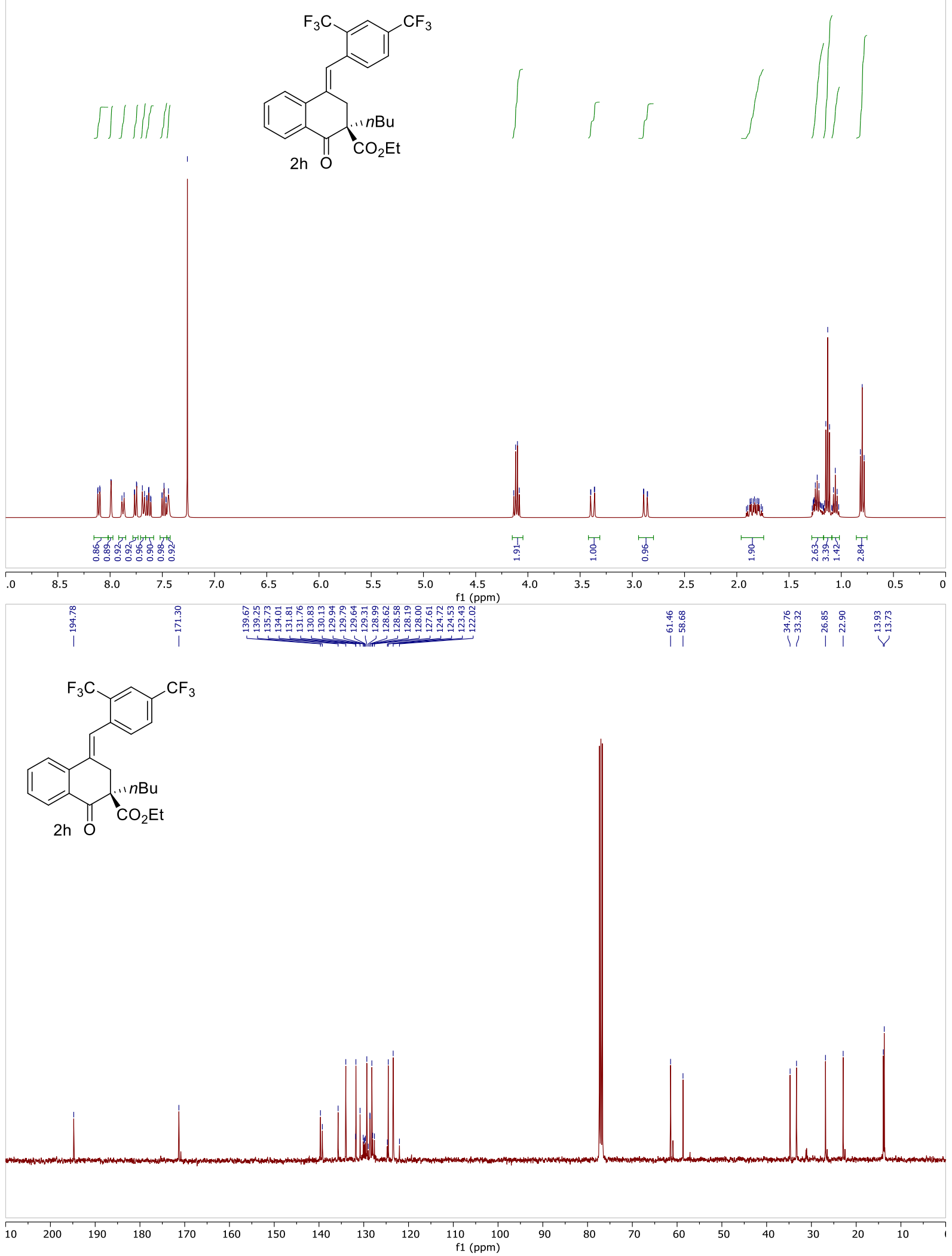




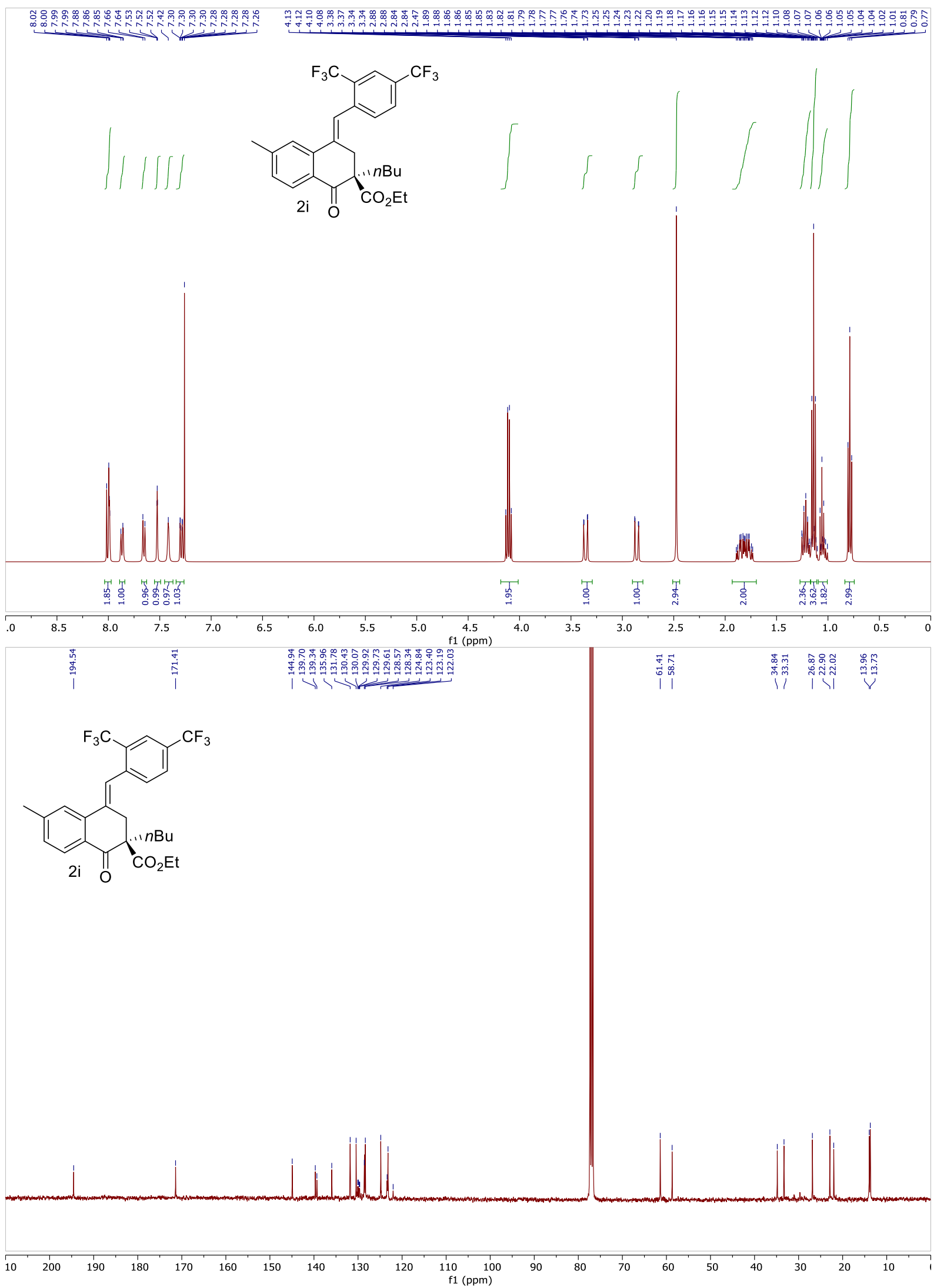




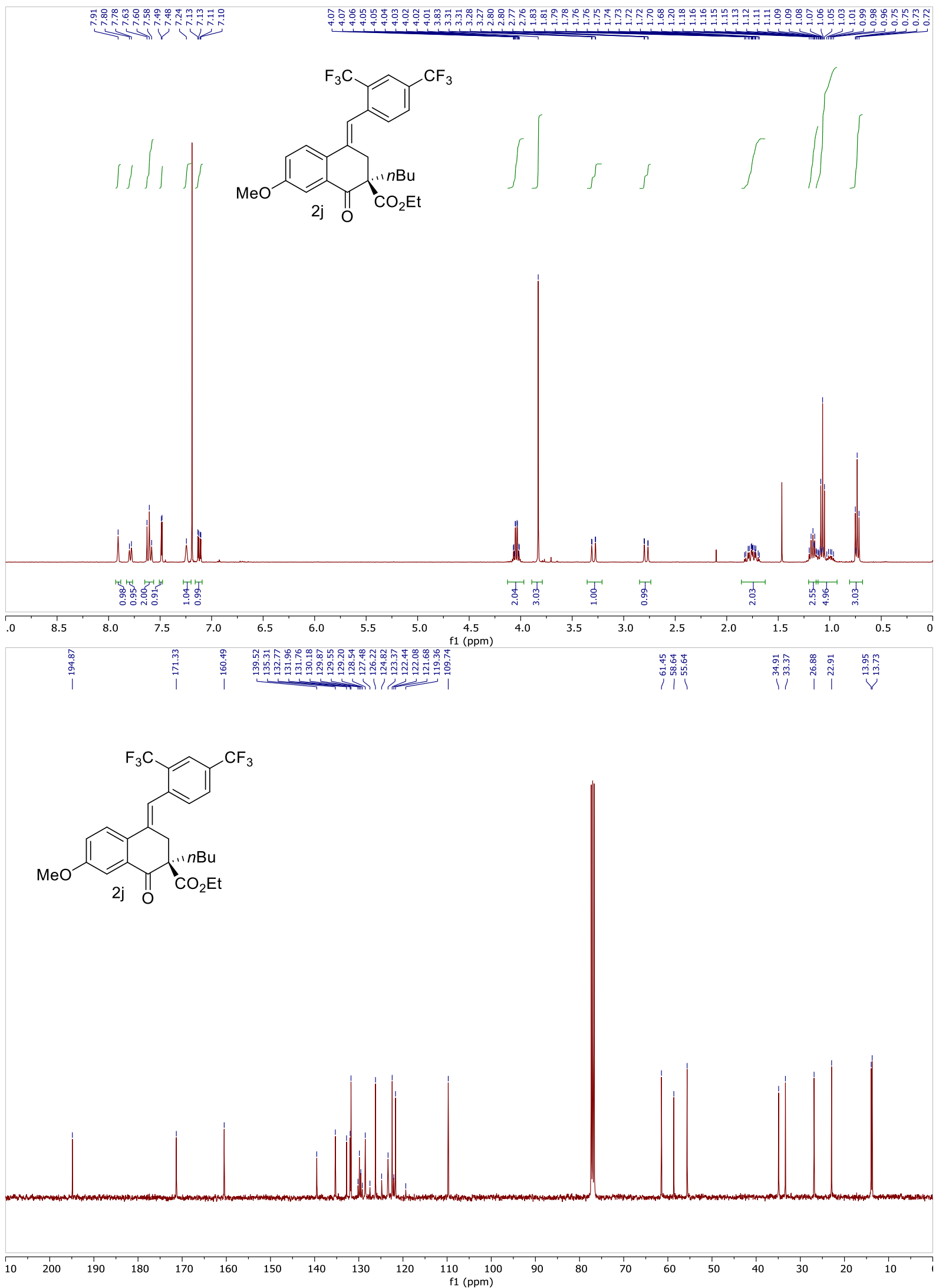




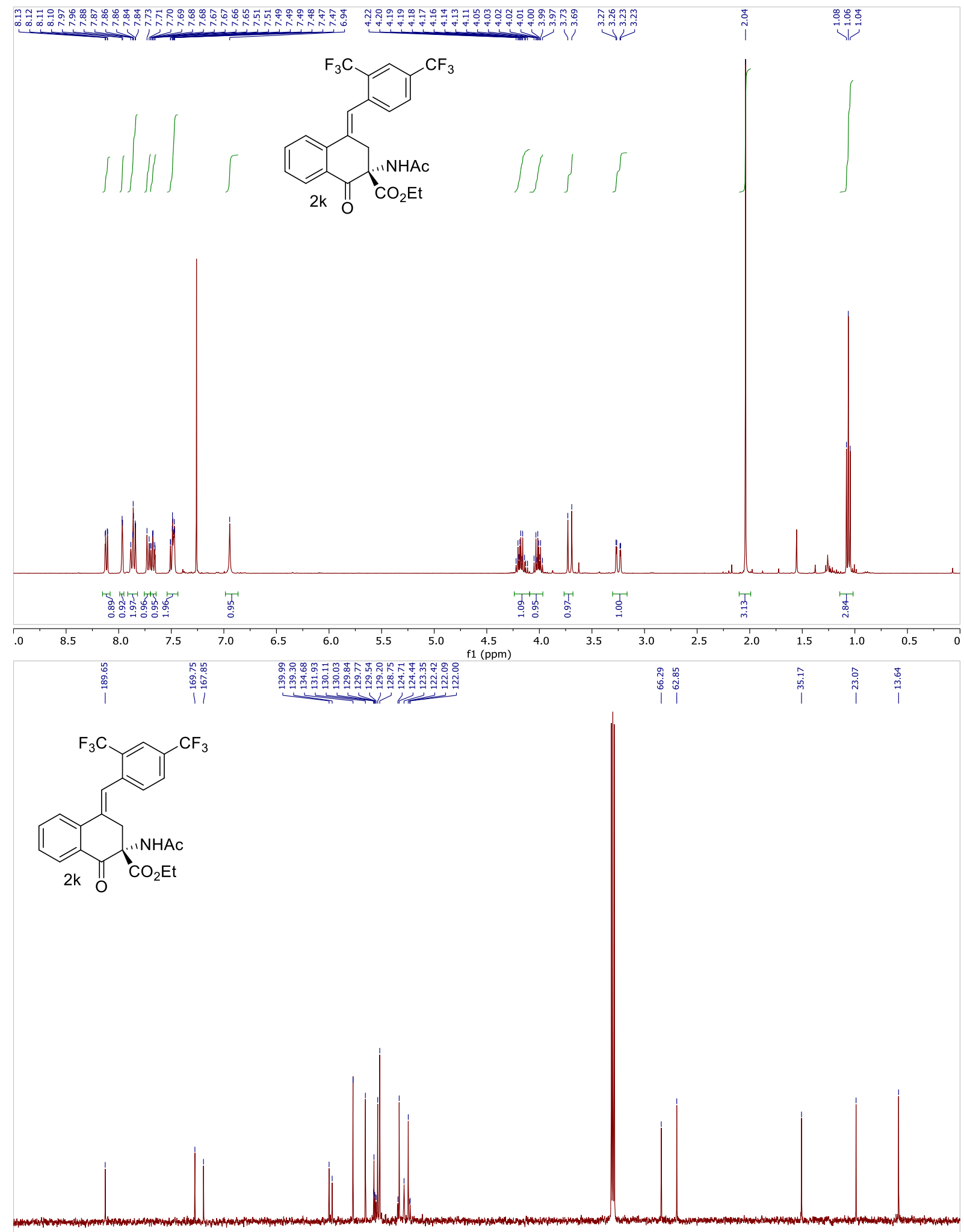

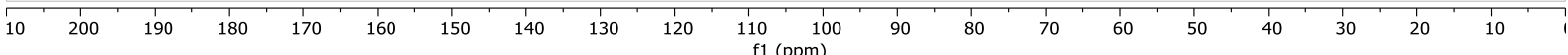




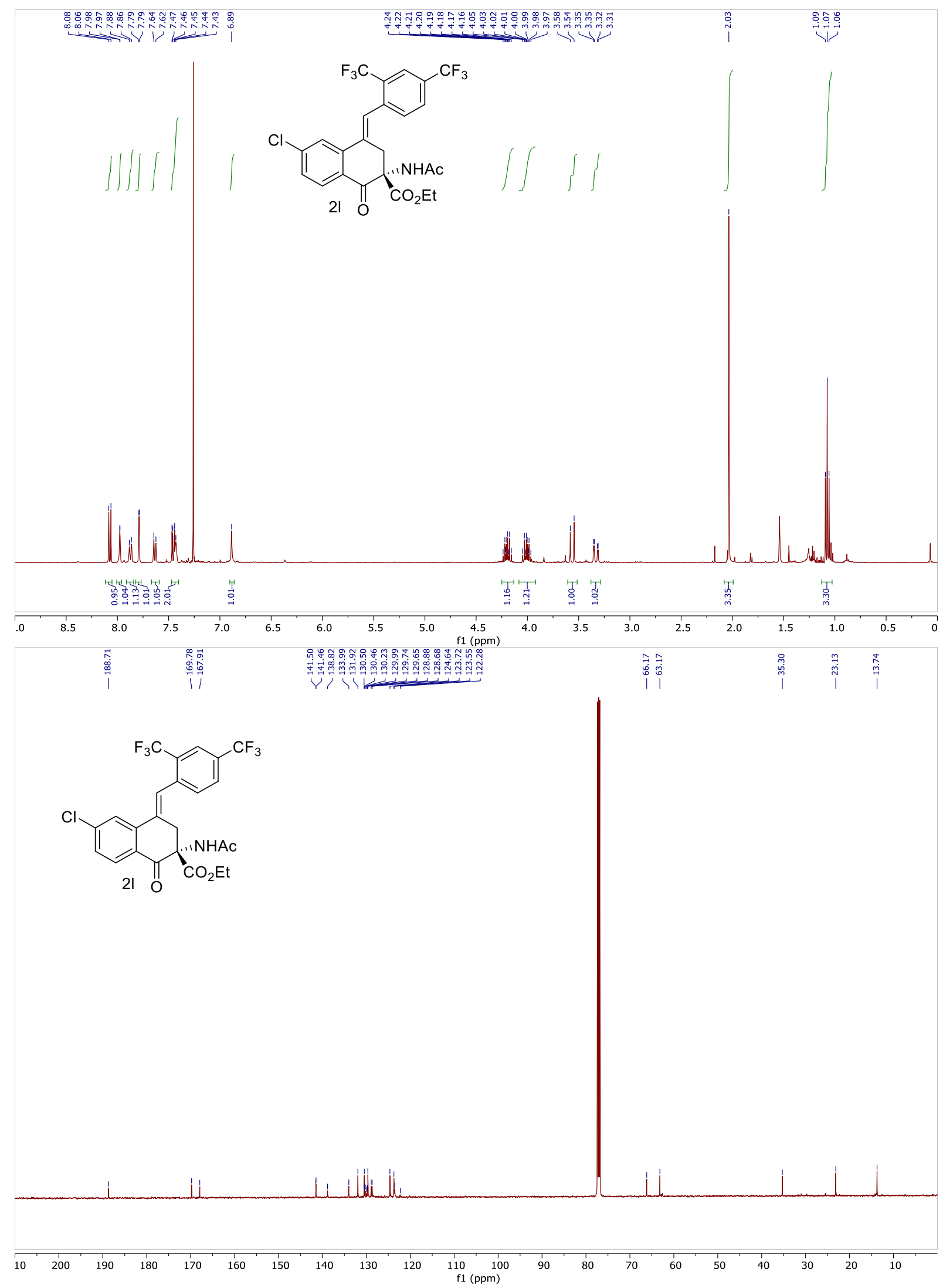




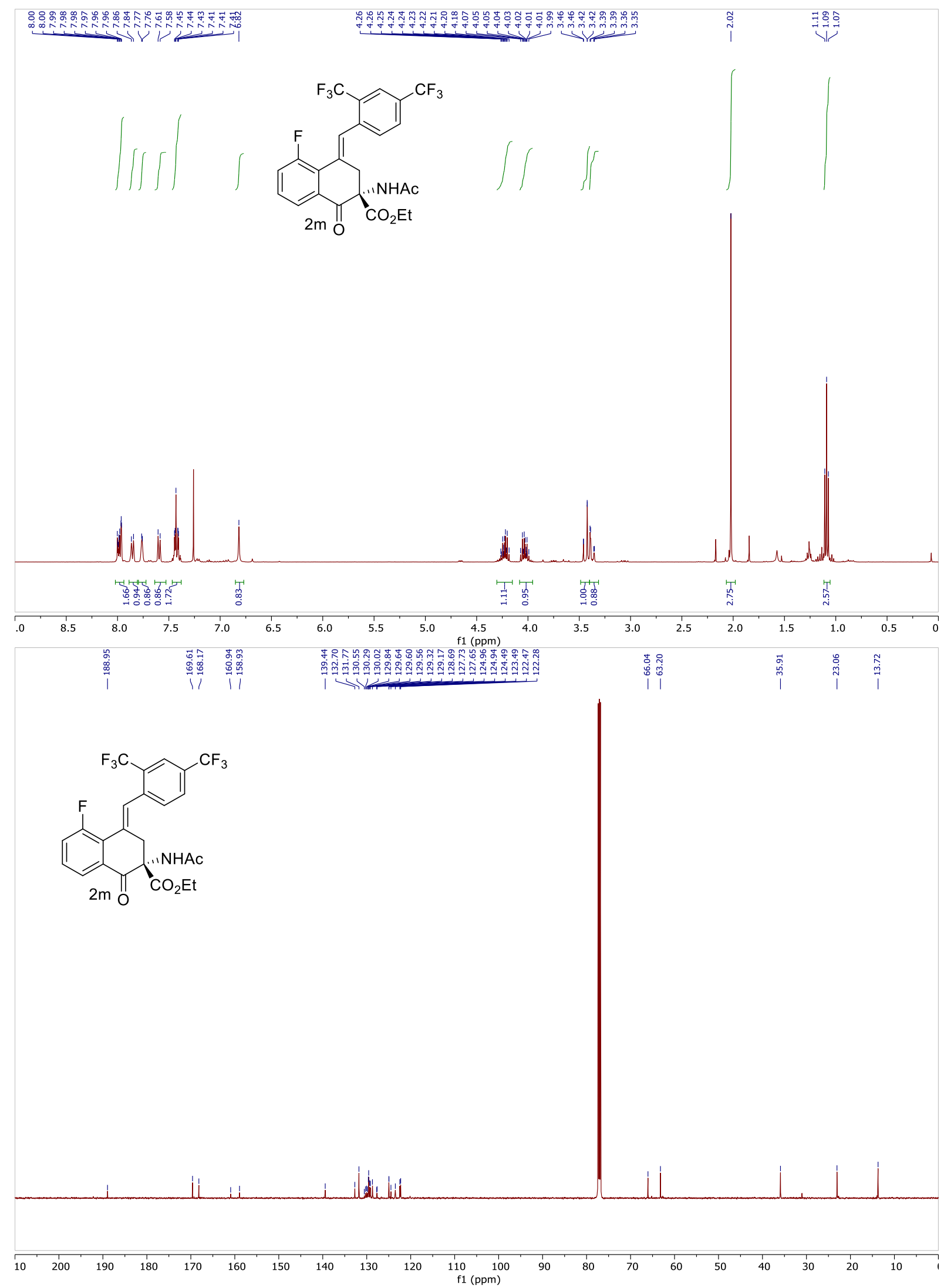




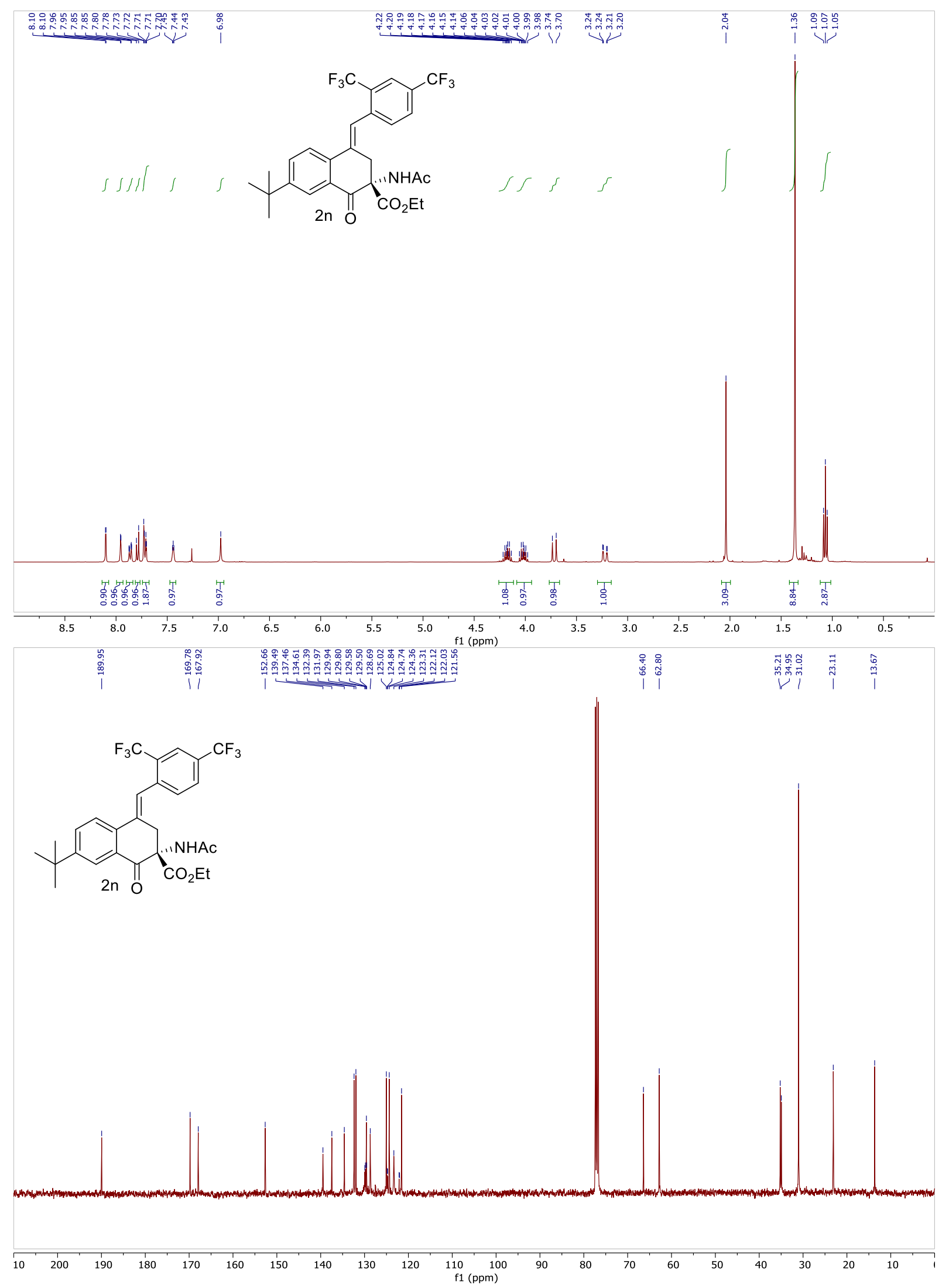




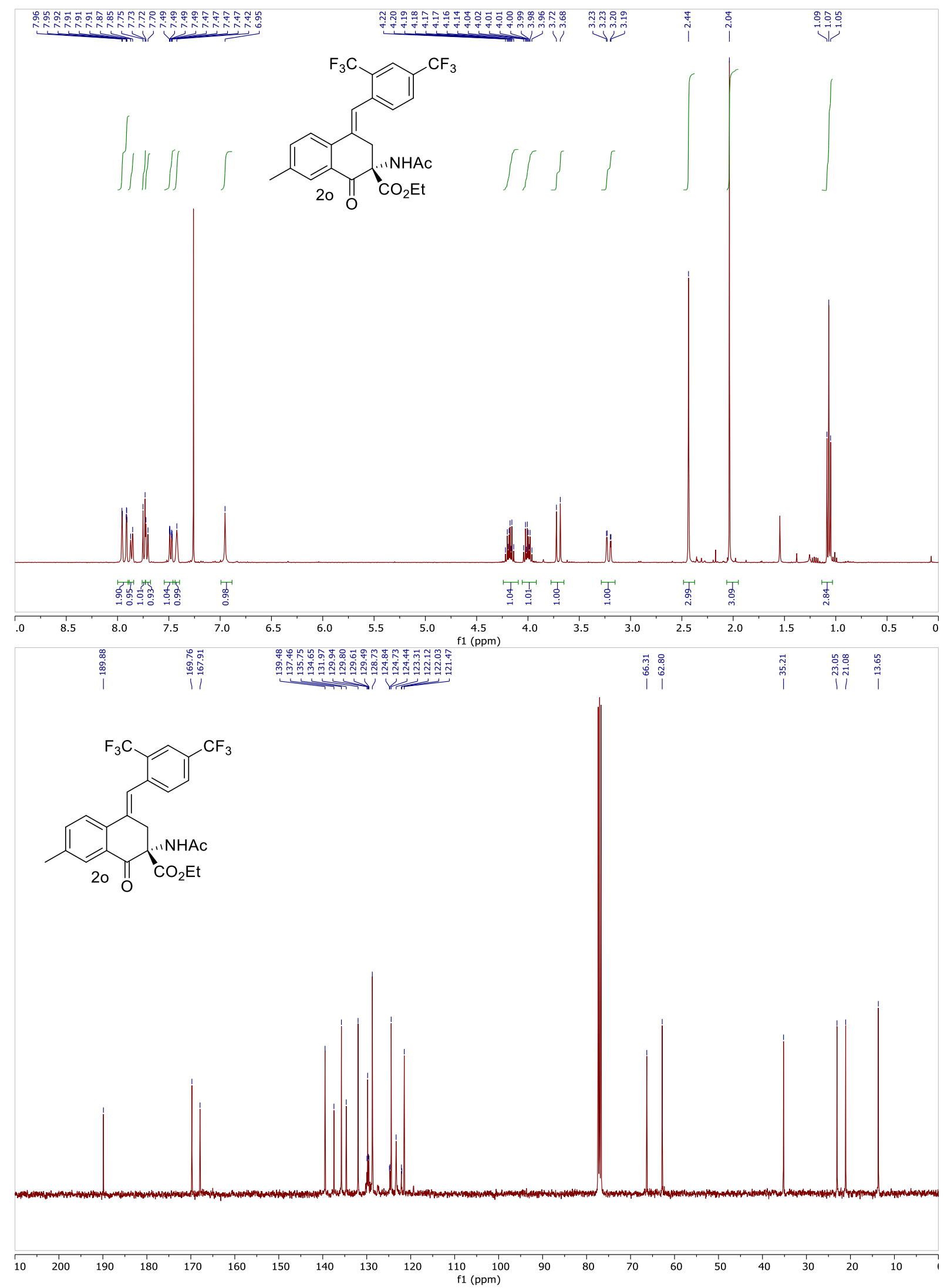




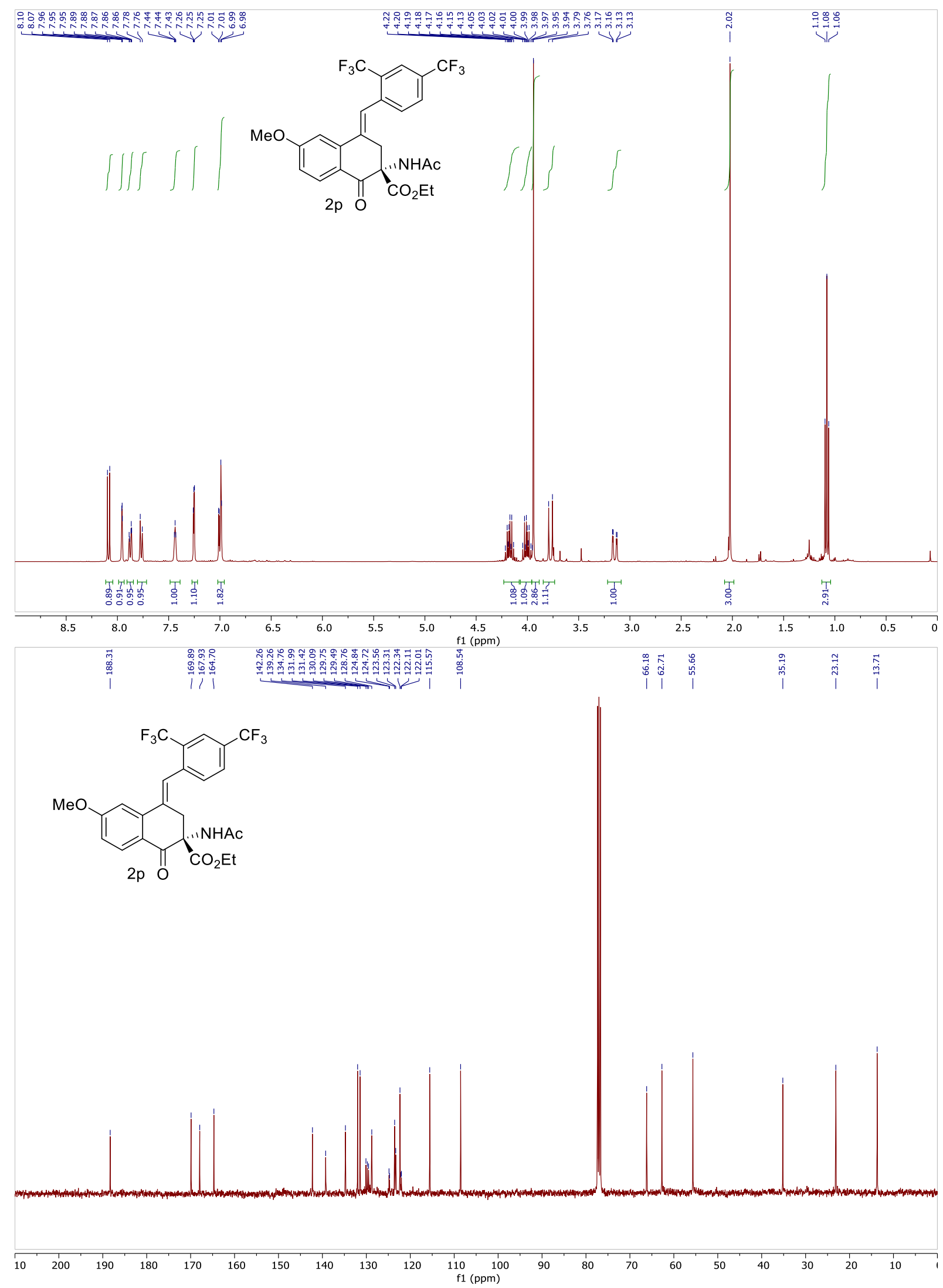




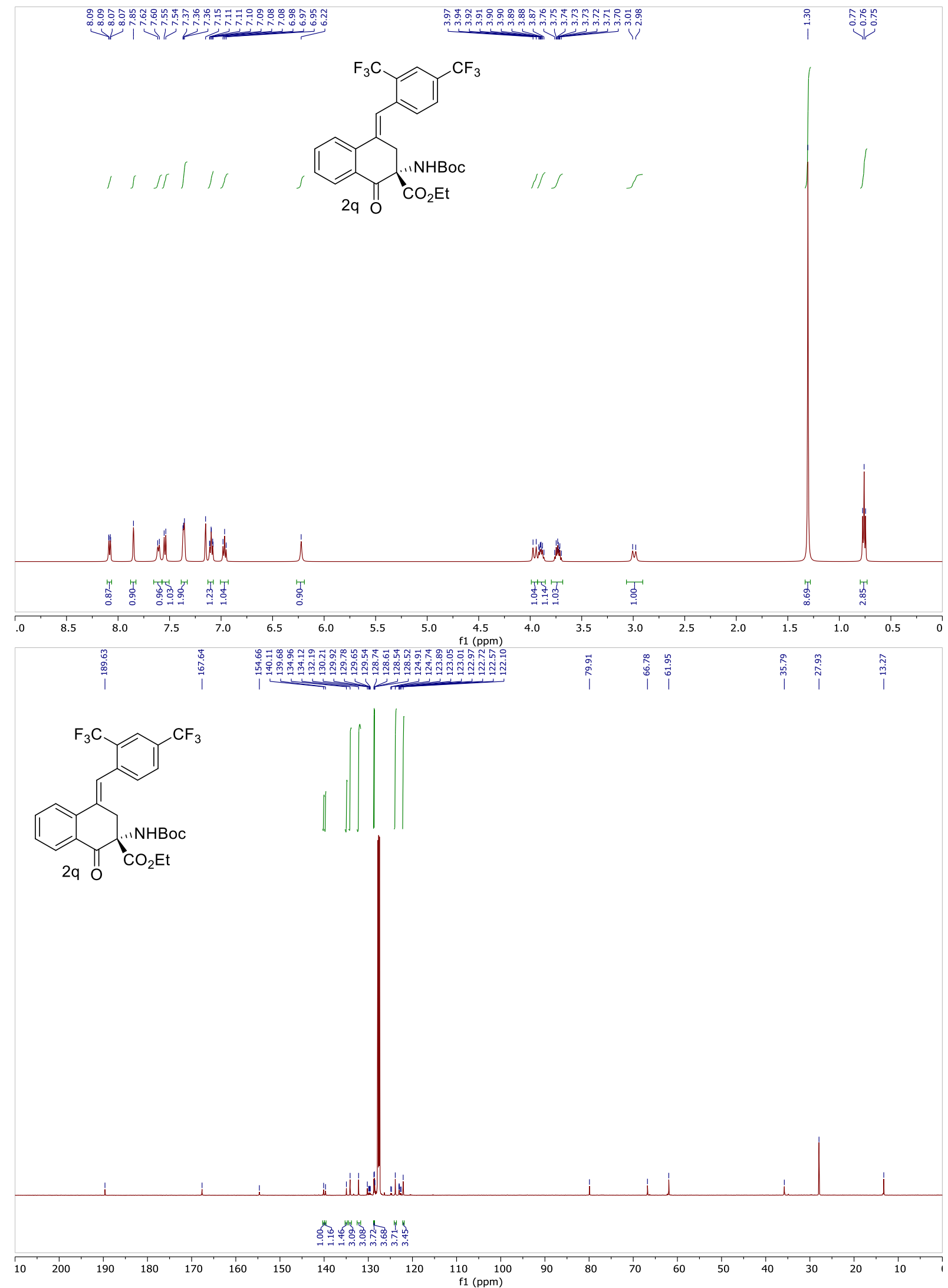




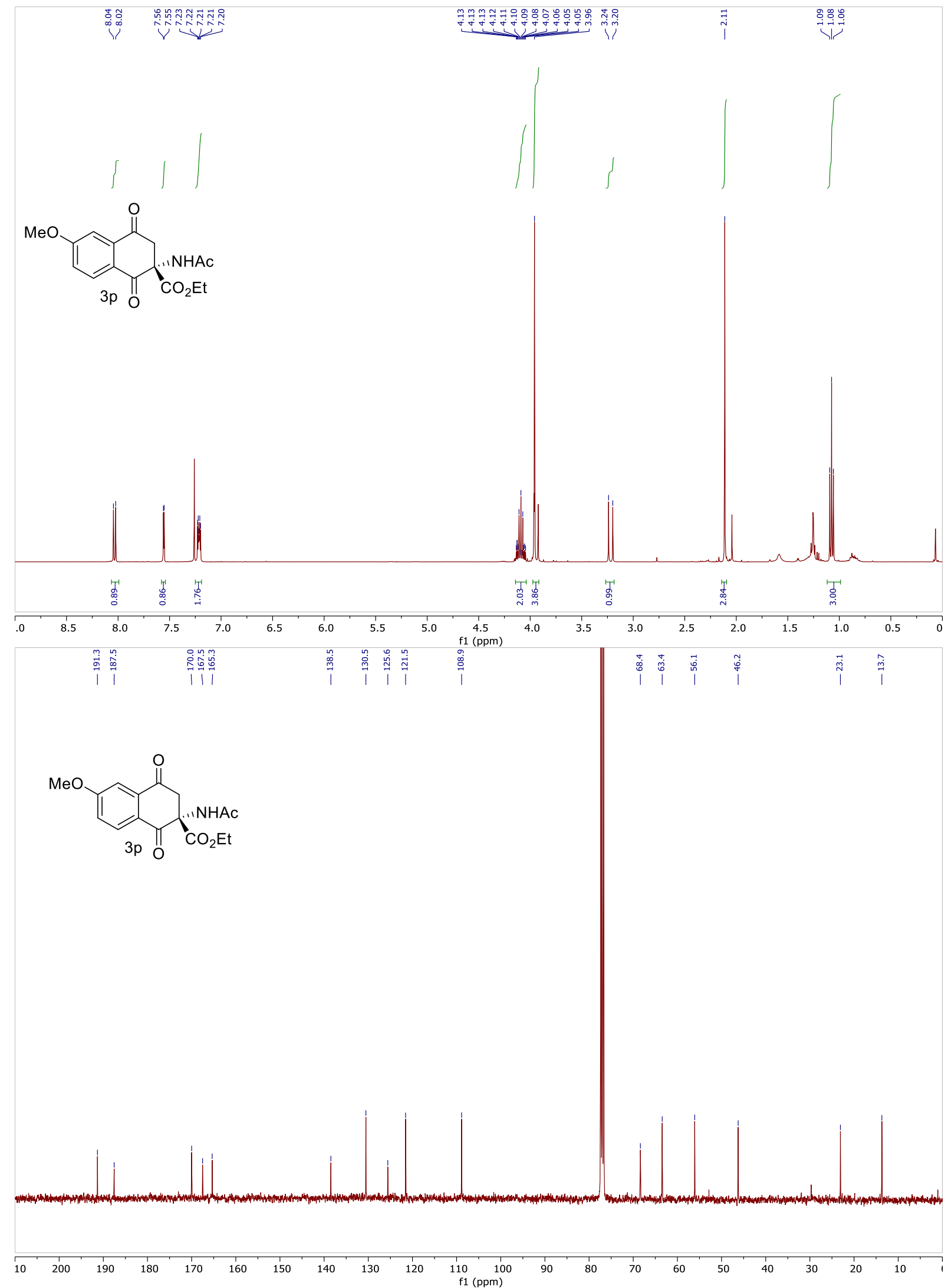

